Florida International University FIU Digital Commons

FIU Electronic Theses and Dissertations

University Graduate School

3-16-2015

\title{
Development of New Structural Health Monitoring Techniques
}

Hadi Fekrmandi

PhD Student, MME department, hfekr001@fiu.edu

DOI: $10.25148 /$ etd.FI15032180

Follow this and additional works at: https://digitalcommons.fiu.edu/etd

Part of the Acoustics, Dynamics, and Controls Commons, Electro-Mechanical Systems

Commons, and the Manufacturing Commons

\section{Recommended Citation}

Fekrmandi, Hadi, "Development of New Structural Health Monitoring Techniques" (2015). FIU Electronic Theses and Dissertations. 1863.

https://digitalcommons.fiu.edu/etd/1863

This work is brought to you for free and open access by the University Graduate School at FIU Digital Commons. It has been accepted for inclusion in FIU Electronic Theses and Dissertations by an authorized administrator of FIU Digital Commons. For more information, please contact dcc@fiu.edu. 


\title{
FLORIDA INTERNATIONAL UNIVERSITY
}

Miami, Florida

\section{DEVELOPMENT OF NEW STRUCTURAL HEALTH MONITORING TECHNIQUES}

\author{
A dissertation submitted in partial fulfillment of \\ the requirements for the degree of \\ DOCTOR OF PHILOSOPHY \\ in \\ MECHANICAL ENGINEERING \\ by
}

Hadi Fekrmandi

2015 
To: Dean Amir Mirmiran

College of Engineering and Computing

This dissertation, written by Hadi Fekrmandi, and entitled Development of New Structural Health Monitoring Techniques, having been approved in respect to style and intellectual content, is referred to you for judgment.

We have read this dissertation and recommend that it be approved.

Jean H. Andrian

Yiding Cao

Bilal El-Zahab

Igor Tsukanov

Ibrahim Nur Tansel, Major Professor

Date of Defense: March 16, 2015

The dissertation of Hadi Fekrmandi is approved.

$\begin{array}{r}\text { Dean Amir Mirmiran } \\ \text { College of Engineering and Computing } \\ \hline \begin{array}{r}\text { Dean Lakshmi N. Reddi } \\ \text { University Graduate School }\end{array}\end{array}$

Florida International University, 2015 
(C) Copyright 2015 by Hadi Fekrmandi

All rights reserved. 


\section{DEDICATION}

To my wife Arlen and my parents, Ali and Zahra. 


\section{ACKNOWLEDGMENTS}

First, I would like to express my deepest gratitude to my advisor, Dr. Ibrahim Nur Tansel, for his support, motivation, patience and guidance through the entire course of my PhD research.

My sincere gratitude is extended to my disseration committee, Dr. Jean $\mathrm{H}$. Andrian, Dr. Yiding Cao, Dr. Bilal El-Zahab and Dr. Igor Tsukanov for their presence in the committee and their invaluable heartening support.

I wish to thank MME’s respected graduate program coordinator, Dr. Cesar Levy. He has been helpful and supportive with all of the PhD program's steps and milestones. I also appreciate Mechanical and Materials Department at Florida International University for the graduate assistantship position, which was my major financial support.

I sincerely acknowledge the University Graduate School at Florida International University for providing support for this research in the form of Dissertation Year Fellowship (DYF) award.

I wholeheartedly appreciate Mr. Richard-Todd Zicarelli’s help in preparing the test pieces for my research at the Engineering Manufacturing Center (EMC) for his help.

I gratefully thank the research group members in mechatronics research laboratory: Amin Baghalian, Shervin Tashakori, Sebastian Rojas Neva, David Meiller, Kyle Lindsay, Felipe Cardozo Arcaro, Luis Sanchez, Kathleen Oyola, Joshua Gonzalez, Nikko Miniello, Abdullah Alsenawi, Michael Wolff, Gurunath Ramanathan and Javier Rojas. 


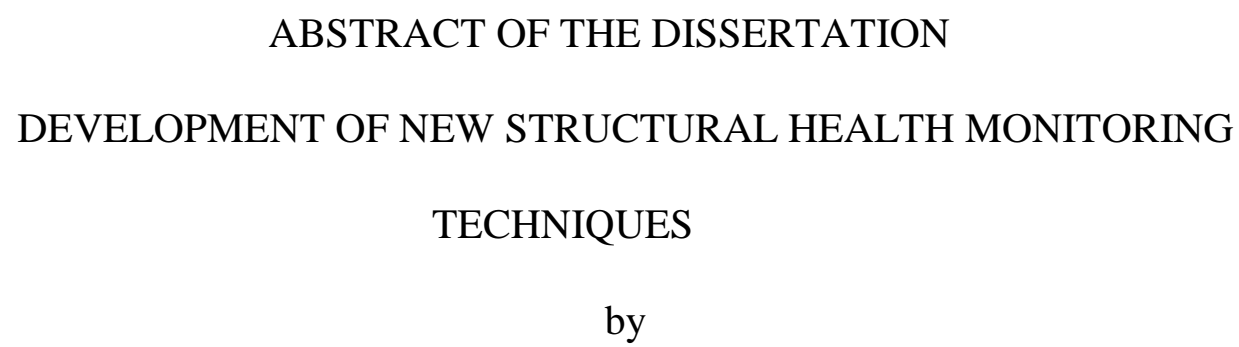

Hadi Fekrmandi

Florida International University, 2015

Miami, Florida

Professor Ibrahim Nur Tansel, Major Professor

During the past two decades, many researchers have developed methods for the detection of structural defects at the early stages to operate the aerospace vehicles safely and to reduce the operating costs. The Surface Response to Excitation (SuRE) method is one of these approaches developed at FIU to reduce the cost and size of the equipment. The SuRE method excites the surface at a series of frequencies and monitors the propagation characteristics of the generated waves. The amplitude of the waves reaching to any point on the surface varies with frequency; however, it remains consistent as long as the integrity and strain distribution on the part is consistent. These spectral characteristics change when cracks develop or the strain distribution changes. The SHM methods may be used for many applications, from the detection of loose screws to the monitoring of manufacturing operations.

A scanning laser vibrometer was used in this study to investigate the characteristics of the spectral changes at different points on the parts. The study started with detecting a load on a plate and estimating its location. The modifications on the part with manufacturing operations were detected and the Part-Based Manufacturing Process 
Performance Monitoring (PbPPM) method was developed. Hardware was prepared to demonstrate the feasibility of the proposed methods in real time.

Using low-cost piezoelectric elements and the non-contact scanning laser vibrometer successfully, the data was collected for the SuRE and PbPPM methods. Locational force, loose bolts and material loss could be easily detected by comparing the spectral characteristics of the arriving waves. On-line methods used fast computational methods for estimating the spectrum and detecting the changing operational conditions from sum of the squares of the variations. Neural networks classified the spectrums when the desktop - DSP combination was used. The results demonstrated the feasibility of the SuRE and PbPPM methods. 


\section{TABLE OF CONTENTS}

CHAPTER

PAGE

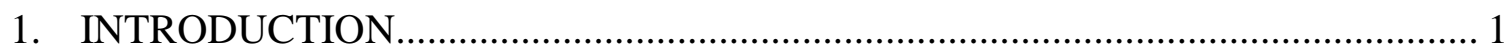

1.1. Structural Health Monitoring ................................................................... 1

1.2. Rayleigh-Lamb Surface Guided Waves......................................................... 2

1.3. Electromechanical Impedance Method.............................................................. 5

1.4. Manufacturing Process Monitoring …..................................................... 9

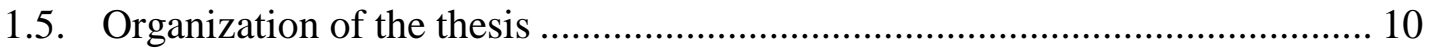

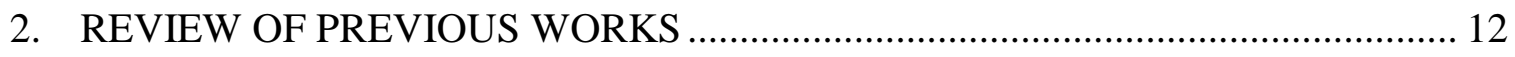

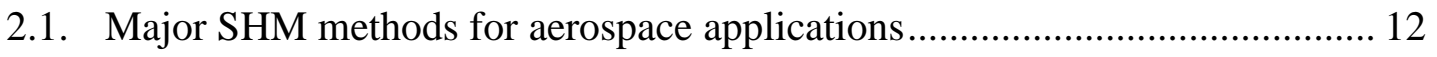

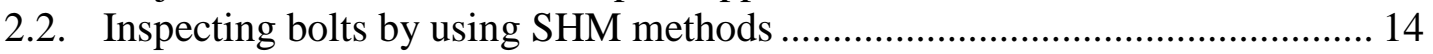

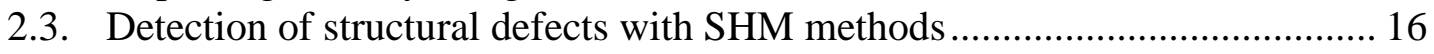

2.4. Automated interpreting SHM signals with neural networks .......................... 20

2.5. Monitoring the performance of machining operations ................................. 21

3. SURFACE RESPONSE TO EXCITATION (SURE) METHOD ............................ 25

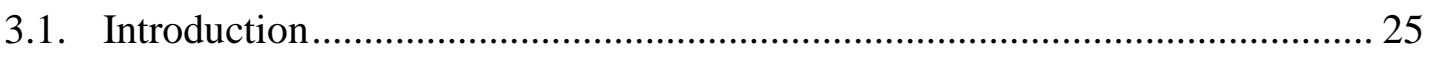

3.2. Excitation and sensing of the SuRE method.............................................. 26

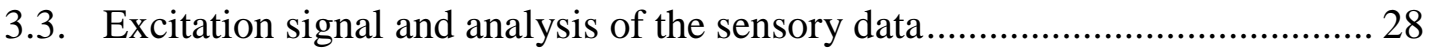

3.4. Computational methods for calculation of the spectrum of the signal ........... 32

3.4.1. Fast Fourier Transform .......................................................... 32

3.4.2. The Teager-Kaiser Algorithm.................................................. 33

3.4.3. The Goertzel Algorithm............................................................. 33

3.4.4. Sine Wave RMS and Mean Value Methods .................................... 33

3.5. Signal Processing for comparing the response magnitudes............................. 34

3.5.1. Sum of square of differences (SSDs) .......................................... 34

3.5.2. Sum of square of differences for multiple sensing point .................. 35

3.5.3. Normalized sum of square of differences (NSSDs) ........................ 36

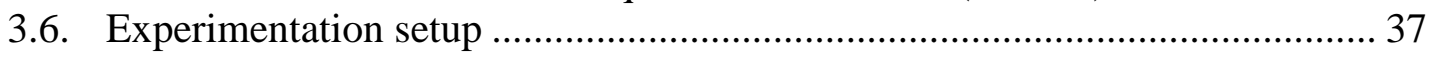

3.6.1. Implementing the SuRE method with Digital Signal

Processors (DSP): ............................................................................ 40

3.6.2. Implementing the SuRE method with spectrum analyzer: .............. 42

3.6.3. Monitoring with a scanning laser vibrometer

(Laser Doppler Vibrometer): ............................................................... 43

4. SIMULATION OF THE SURFACE RESPONSE TO EXCITATION

METHOD FOR STRUCTURAL HEALTH MONITORING ...................................... 44

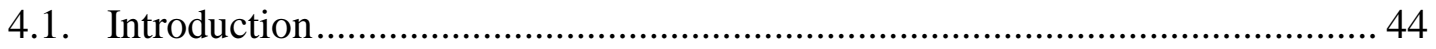


4.2. Implementation of SuRE Algorithm.....

4.3. Use of COMSOL Multi-physics for Sure-based Manufacturing

Process Performance Monitoring Method................................................................. 45

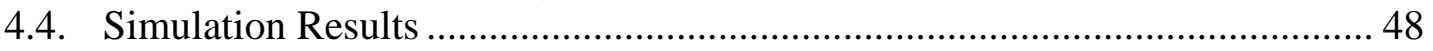

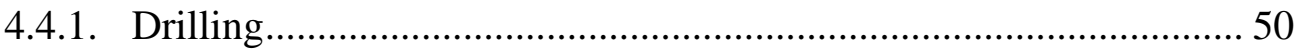

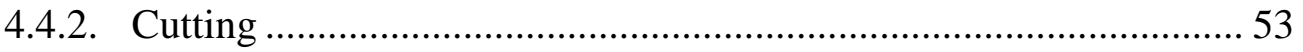

4.4.3. Milling ………………………................................................. 56

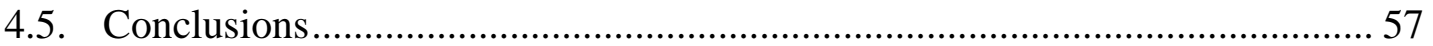

5. IMPLEMENTATION OF THE SURE METHOD FOR

STRUCTURAL HEALTH MONITORING (SHM) APPLICATIONS ............................ 58

5.1. Monitoring the Load Condition of a Beam Remotely by Using Scanning

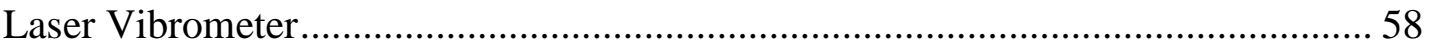

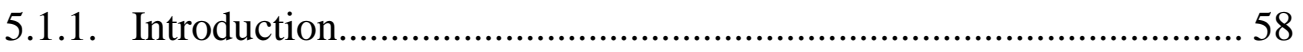

5.1.2. Experimental setup ………………………….............................. 58

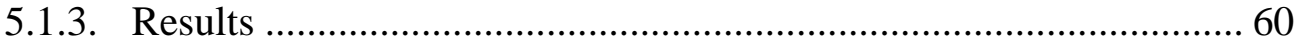

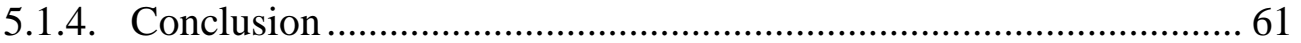

5.2. Inspecting Integrity of a Multi-Bolt Robotic Arm Using a Scanning

Laser Vibrometer and Implementing the Surface Response to Excitation

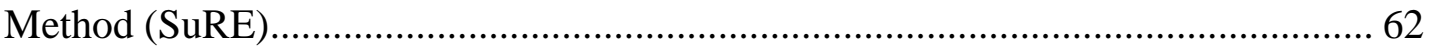

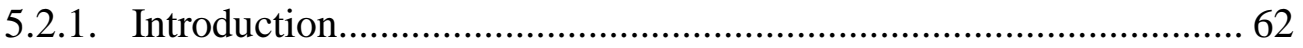

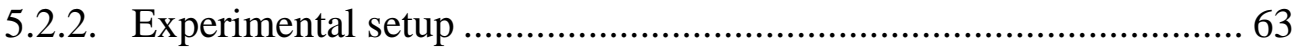

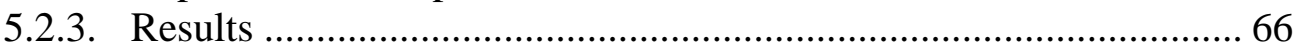

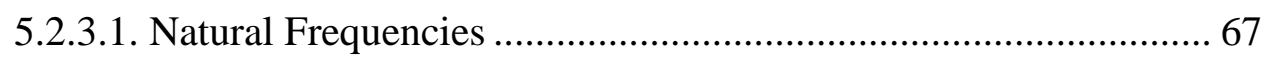

5.2.3.2. Detection of Loose Bolts in Linear Grid...................................... 68

5.2.3.3. Detection of Loose Bolts in Two Dimensional Grid .................... 72

5.2.3.4. Detection of Loose Bolts in Full Arm Grid .................................. 74

5.2.3.5. Detection of Multiple Loose Bolts in Full Arm Grid ................... 76

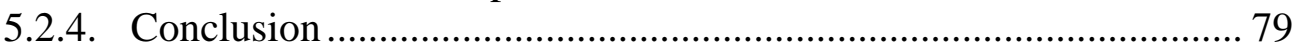

5.3. Surface Response to Excitation Method for Structural Health

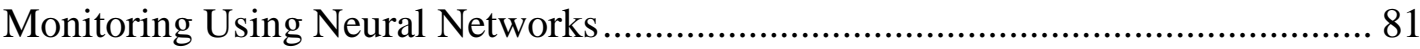

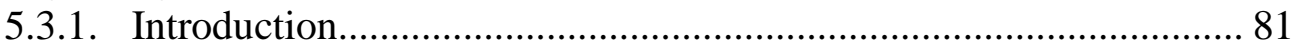

5.3.2. Experimental Setup............................................................................ 82

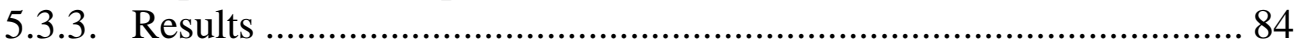

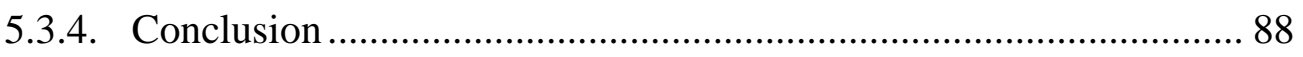

6. IMPLEMENTATION OF SURE METHOD FOR PART-BASED

MACHINING PROCESS PERFORMANCE MONITORING......................................... 89

6.1. Laser scanning vibrometer for remote process performance monitoring of automated manufacturing operations ................................................... 89

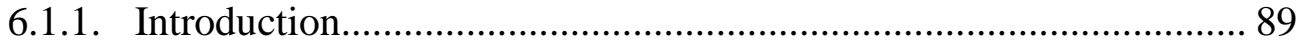

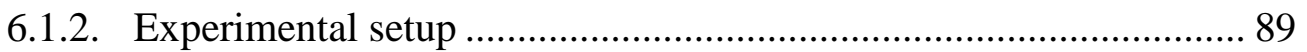

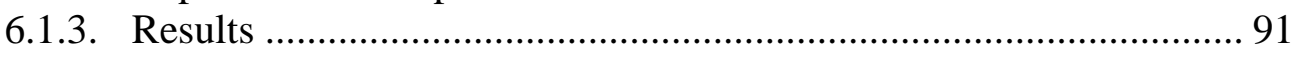




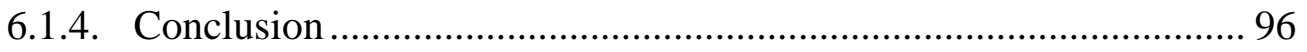

6.2. An Experimental Investigation of Part-based Process Performance

Monitoring Method for Machining Process Monitoring............................................ 97

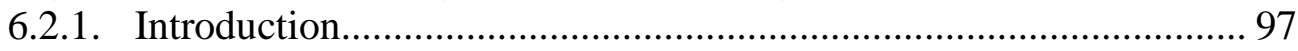

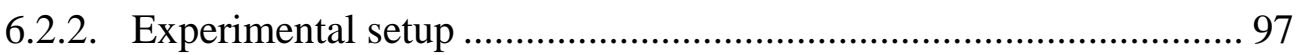

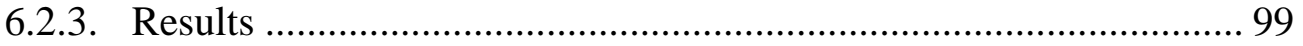

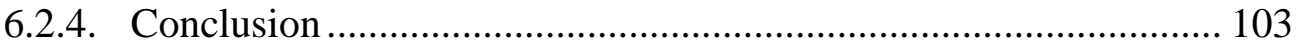

6.3. A non-contact method for part-based process performance monitoring

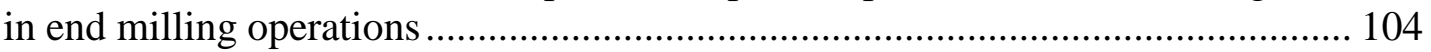

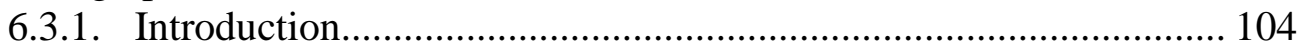

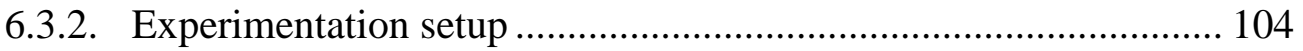

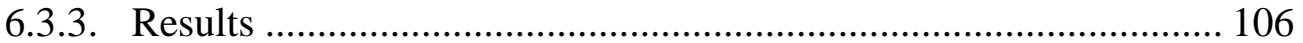

6.3.3.1. Study of milling length .............................................................. 109

6.3.3.2. Study of milling depth ............................................................. 110

6.3.3.3. Study of milling width ............................................................... 111

6.3.4. Conclusion ..................................................................................... 112

6.4. Surface Response to Excitation Method for Part-Based Manufacturing

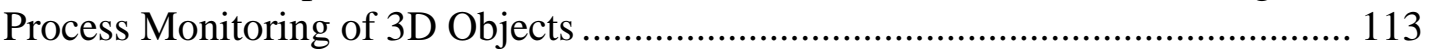

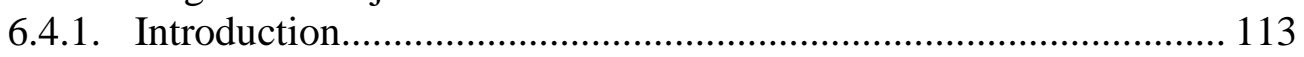

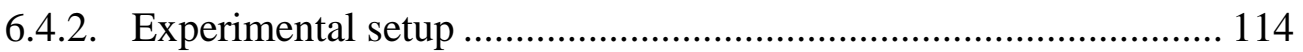

6.4.2.1. Piezoelectric-Based Implementation ........................................... 114

6.4.2.2. Laser Vibrometer-Based Implementation................................... 115

6.4.2.3. Embedded Device-Based Implementation.................................. 116

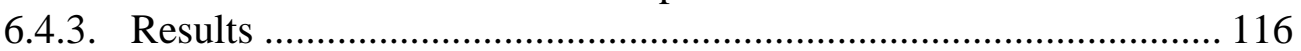

6.4.3.1. Contact Sensing Piezoelectric-based implementation ................ 116

6.4.3.2. Non-Contact Laser vibrometer-based implementation................ 120

6.4.3.3. DSP device-based implementation ........................................... 121

6.4.4. Conclusion ..................................................................................... 122

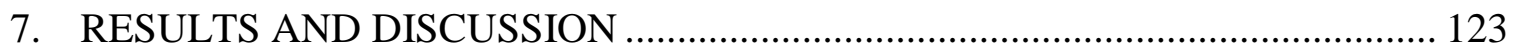

7.1. Investigation of Computational Efficiency and Validity of the

Surface Response to Excitation Method ................................................................. 123

7.1.1. Comparison of magnitude estimation methods ................................ 124

7.1.2. Implementation of the SuRE method with a DSP system ............... 125

7.1.3. Inspection of composite coating by using the SuRE method .......... 126 7.1.3.1. Implementation of the SuRE method by using scanning

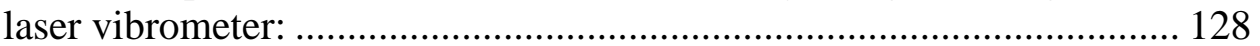

7.2. Effectiveness of tested approaches ............................................................... 129

7.2.1. Comparison of effectiveness of laser scanning vibrometer, DSP system and spectrum analyzer for implementing SuRE method........ 129

7.2.2. Uncertainty of Measurements.......................................................... 132

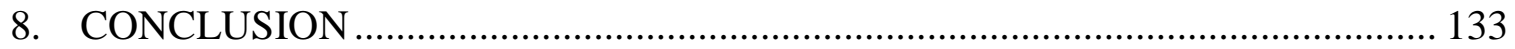


9. REFERENCES.

VITA 150 


\section{LIST OF TABLES}

TABLE

PAGE

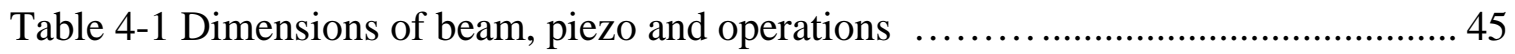

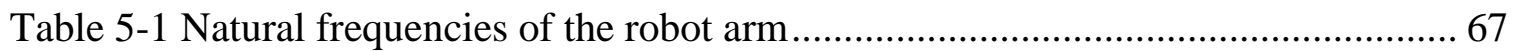

Table 5-2 Output of neural network based on load condition .................................... 83

Table 5-3 Result comparison between both training algorithms ................................. 85

Table 6-1: Drill hole diameters at during a 6 step drilling process............................... 91

Table 6-2 Cutter diameter in drilling process and Depth of cut in cutting process .......... 99

Table 6-3 HSS flat head milling heads being used for the experiments of this

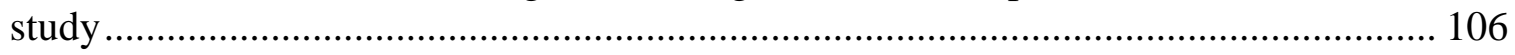

Table 6-4 HSS flat head milling heads being used for the experiments of this study.....

Table 6-5 Sum of squared differences before and after damage

Table 7-1 Comparison of the normalized sum of squares of difference for the Laser vibrometer, dsPIC33F and spectrum analyzer .

Table 7-2 Uncertainty in the form of percentage of variation in measurements calculated using root mean square (RMS) method.... 


\section{LIST OF FIGURES}

FIGURE

PAGE

Figure 1-1 Lamb wave modes (a) Symmetric (b) Anti-symmetric.................................. 3

Figure 1-2 Dispersion curves for symmetric and anti-symmetric lamb waves [1]........... 4

Figure 1-3 Schematic of indirect measurement of mechanical impedance of structure using electromechanical impedance (EMI) method ..................................... 6

Figure 1-4 The contraction and expansion of PZT material due to direct

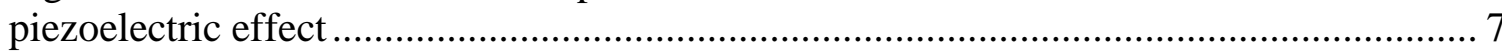

Figure 1-5 scanning laser vibrometer structure and sensing mechanism ....................... 8

Figure 1-6 The framework of tool condition monitoring [114] ................................... 9

Figure 1-7 part-based process performance monitoring (PbPPM)[36] ........................... 10

Figure 2-1 Essential steps in guided-wave-based structural health monitoring [41]....... 18

Figure 2-2 Wireless embedded device developed by Lynch et al. [137]........................ 20

Figure 3-1 Schematic of the Surface Response to Excitation (SuRE) method................. 27

Figure 3-2 (a) The spectrums of a point on the grid for two successive no load scans (b) Spectrums of a point on the grid before and after the load was applied .................... 28

Figure 3-3 FFT of sweep sine wave excitation and corresponding peak hold spectrum .. 29

Figure 3-4 (a) Received signal in the time domain (b) Zoom of the received signal

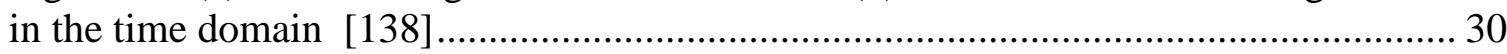

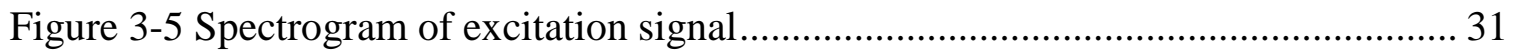

Figure 3-6 The change of squares of the differences (SSD’s) in drilling operation ......... 32

Figure 3-7 An example of the frame used to hold the plate for laser scan ..................... 39

Figure 3-8 3/4” diameter APC PZT model D-.750"-2MHz-850 WFB............................. 39

Figure 3-9 The experimental setup for the SuRE method using a signal analyzer and a DSP circuit 
Figure 3-10 Embedded device for the SuRE method (a) power source, (b) dsPIC33f, (c) DDS-60 (e) OPAMP (d) UART (e) R232-USB adapter ............................................... 41

Figure 3-11 Stanford Research Systems (SRS)-SR780 spectrum analyzer....................... 42

Figure 3-12 PolyTech PSV-400 Laser Doppler Vibrometer experimental set up; (a) Vibrometer Controller (b) Scanning Head (c) PSV software (d) the SuRE method color-map (e) Aluminum plate. 43

Figure 4-1 Schematic of aluminum beam with PZT exciter, scan probes and drilling, cutting and milling operations 45

Figure 4-2 Aluminum beam model with a PZT element in center; drilling, cutting and milling are simulated.

Figure 4-3 Meshed model with free tetrahedral elements .................................................. 47

Figure 4-4 Accelerations vs. frequency in spectrum from a probe point........................... 48

Figure 4-5 (a) Spectrums of before and after the drill hole from a probe in $2^{\text {nd }}$ column

(b) Spectrums of before and after the drill hole from a probe in $6^{\text {th }}$ column 49

Figure 4-6 The drilling surface displacements solution for the 1st frequency step; $20[\mathrm{kHz}]$. 50

Figure 4-7 The drilling surface displacements solution for the 3rd frequency step; 22[kHz]. 51

Figure 4-8 Bar diagram of SSD values vs. scan points for drilling. ................................. 52

Figure 4-9 Color-map of SSD values vs. scan surface for drilling................................... 52

Figure 4-10 The cutting surface displacements solution for the 6th frequency step; 25[kHz]; waves passed thought cut without considerable interaction.

Figure 4-11 The cutting surface displacements solution for the 7th frequency step; $26[\mathrm{kHz}]$; waves reflected from cutting edge.

Figure 4-12 The cutting surface displacements solution for the 15th frequency step; $34[\mathrm{kHz}]$; waves passed thought cut with showing interaction. 55

Figure 4-13 Color-map of SSD values vs. scan surface for cutting.................................. 55

Figure 4-14 Bar diagram of SSD values vs. scan points for cutting .................................. 56 
Figure 4-15 The cutting surface displacements solution for the 14th frequency step; 32[kHz]; waves interacted with milling are mixed with vibrations around piezo ... 56

Figure 4-16 Bar diagram of SSD values vs. scan points for milling. ............................ 57

Figure 5-1(a) Frame (b) Screws to apply load to the beam .......................................... 59

Figure 5-2 Scanning grid; blue is scan points, white is already scanned and brown is deactivated points

Figure 5-3 Location of application of force on the plate and the variation of normalized sum of the squares of the differences.....

Figure 5-4 Experimental schematic 64

Figure 5-5 The robot structure and close up view of the scanned section..... 65

Figure 5-6 Bolted joint on the robot arm with bolt numbers 66

Figure 5-7 FFT spectrum comparison (a) Scan1 with all bolts tight and Scan 2 with all bolts tight (b) Scan1 with all bolts tight and Scan 3 with a loose bolt..... 69

Figure 5-8 Local linear scan grid with eight scanning points specified on both sides of the lower four bolts.

Figure 5-9 Normalized sum of square of differences for linear local scan for (a) before (b) after; single bolt was loosened.

Figure 5-10 Sum of Squared Difference (SSD) values before and after loosening bolt 8.72

Figure 5-11 Bar diagram of normalized sum of square of differences for full arm without any loose bolts 73

Figure 5-12 Bar diagram of normalized sum of squared differences for full arm with two loose bolts 73

Figure 5-13 Contour map of normalized sum of squared differences for full arm scans with two loose bolts; bolt 3 and bolt 5 are loosened.

Figure 5-14 Two-dimensional full arm scan grid with 20 columns and 3 scan points in each column

Figure 5-15 Normalized sum of squared differences for full arm scan; only bolt 8 is loosened. 
Figure 5-16 Contour map of normalized sum of squared differences for loosening bolt 8 76

Figure 5-17 Normalized sum of squared differences for full arm scan; bolt 2 and bolt 7 are loosened

Figure 5-18 Contour map of the normalized sum of square of differences for multiple bolt loosening.

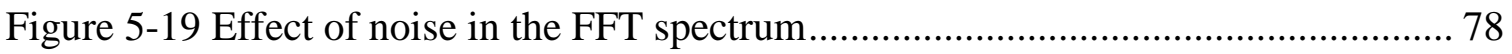

Figure 5-20 Load applied on four points (points 2-5) on a composite beam using a clamp.

Figure 5-21 A typical spectrum built with the time domain data ................................. 84

Figure 5-22 Comparison between spectrums depending of load application point.......... 84

Figure 5-23 Comparison of classification results of both methods ............................... 86

Figure 5-24 Performance of the neural network when (a) the test columns, (b) the test rows were estimated

Figure 5-25 Sum of square of differences between the no load reference spectrum and load spectrums 88

Figure 6-1 schematic of experimentation process

Figure 6-2 3×14 scan grid with 42 scan points; Region 1 for cutting (R1) and Region 2 (R2) for drilling 90

Figure 6-3 (a) The cutting process on the aluminum beam and (b) the corresponding contour map of normalized sum of squared differences

Figure 6-4 Normalized sum of squared differences (NSSDs) of scan point [2,6] at during the cutting operation.....

Figure 6-5 Normalized sum of squared differences (NSSDs) of scan point [1,3] at during the cutting operation

Figure 6-6 The drilling process on the aluminum beam and the corresponding contour map of normalized sum of squared differences.

Figure 6-7 Normalized sum of squared differences (NSSDs) of scan point [2.11] at during the drilling operation 95 
Figure 6-8 Schematic of experimentation set up 98

Figure 6-9 scan grid including 35 scan points on a $5 \times 7$ grid with region 1 for drilling (R1), region 2 for cutting (R2), and region 3 for milling (R3). 98

Figure 6-10 (a) Color map diagram of sum of squared of differences for drill process;

(b) three-dimensional graph of SSD values for drilling process. 100

Figure 6-11 values and uncertainty during 8 steps of (a) drilling (b) and cutting process. 101

Figure 6-12 Sum of square of differences for measurement points in column 6 (top) and row 4 (bottom)...

Figure 6-13 contour plots of SSD for (a) step 2 with 0.25 depht and (b) step 6 with 0.75 depth 103

Figure 6-14 Experimentation setup for SuRE system.............................................. 105

Figure 6-15 Scan grid included 50 scan points that were arranged in 5 rows and 10 columns with the piezo element in the middle. 106

Figure 6-16 The coolant and cutting chips are covering the scan points in area close to milling operation. 107

Figure 6-17 $1^{\text {st }}$ Reference spectrum vs. $2^{\text {nd }}$ reference spectrum, 5 (b) $1^{\text {st }}$ reference spectrum vs. $1^{\text {st }}$ milling spectrum 108

Figure 6-18 Contour map of SSD values on the scan area for the (a) 1st milling (b) $2^{\text {nd }}$ milling (c) $3^{\text {rd }}$ milling 109

Figure 6-19 Maximum SSD values on the scan area for the study of milling length (a) 1st milling step (b) 2nd milling step (c) 3rd milling step ........................................... 110

Figure 6-20 Three-dimensional demonstration of SSD values on the scan area ............ 111

Figure 6-21 SSD values versus depth of milling increasing in 3 steps........................ 111

Figure 6-22 7.10 SSD values versus depth of milling increasing in 4 steps.................. 112

Figure 6-23 Laser sensor-based implementation procedure of part-based process performance monitoring.

Figure 6-24 Schematic of aluminum block with five piezoelectric elements bonded on three perpendicular faces and laser scan points 
Figure 6-25 Reference data measured from piezo 2 when piezo 1 is exciting

Figure 6-26 Frequency spectrums data measured after surface damage to block; piezo 2 exciting and piezo 1, 3, 4 and 5 sensing

Figure 6-27 Bar diagram of sum of square of differences from laser data; piezo 3 exciting and piezo 1, 2, 4 and 5 reading

Figure 6-28 Sum of square of differences from laser data; piezo 1 exciting and laser vibrometer measuring data from scan points

Figure 6-29 Frequency spectrums from DSP data; piezo 4 exciting piezo 1, 2, 3, 5 reading.

Figure 6-30 Sum of square of differences from DSP data; piezo 2 exciting and piezo $1,2,3$ and 4 reading.....

Figure 7-1 The frequency response estimation for a sweep sine wave signal of amplitude 1 (left), The frequency response estimation for a simulated 1-DOF system (right).

Figure 7-2 The average magnitude response of the of the tests at the initial condition (Data 13), with the load applied (Data 14), and with the load released (Data 15). The SSD values for the 12 test cases were also calculated (right).

Figure 7-3 One side of the aluminum test beam surface was coated with a fiberglass .. 127

Figure 7-4 The estimated magnitude response (left) and the sum of the squares of the differences at 6 frequency intervals (right). Data 12 and 14 are without and with the coating respectively....

Figure 7-5 Various scans taken with the laser vibrometer looking for localized loads. The load was applied to the bottom right.

Figure 7-6 Comparison of sum of squares of differences, Column 1-6 is corresponding to clamp applying load on point P1-P6; Schematic of load tests for comparison (left), data from spectrum analyzer (right).

Figure 7-7 Normalized sum of squares of differences; load applied with the clamp to points 1-6 respectively (left P1-P6), Load removed from the point 1-6 (right P1-P6)131 


\section{INTRODUCTION}

\subsection{Structural Health Monitoring}

Structural health monitoring (SHM) is a growing field that attracts a considerable research effort from scientists all over the world. Only in the United States are the maintenance and scheduled inspection of aging civil structures, industrial equipment and machinery costs more than $\$ 200$ billion every year [1]. Structural health monitoring has multiple aspects and applications.

In condition-based monitoring (CBM), the goal is to replace the schedule-based maintenance with condition-based maintenance. This way, unnecessary maintenance sessions and consequent costs involved with the shutdown of the system would be eliminated. A new trend is to include the sensors and embedded monitoring systems within the structure from design and manufacturing stages. Such approach for health monitoring not only could reduce the installation costs of the SHM system and increase the reliability and safety, but also optimize the final weight, size and cost of systems. In prognostics and health management (PHM), the purpose is to diagnose the pattern of creation and development of defect in structure through proper data analysis and interpretation. This knowledge is used to predict the remaining life of the engineering structures. Such approach is especially important for aging structures and allows many civil and aerospace structures to stay in service beyond their original design life cycle.

SHM methods may be divided in two main groups: passive and active methods [3]. Passive-methods use one or multiple sensors to monitor temperature, acceleration, stress or other physical characteristics to identify the problems. The Impedance $[4,5]$ and Lamb-wave $[1,6]$ methods are very widely studied active SHM methods. Active-methods 
excite the system with vibrations, heat, forces or other physical inputs. Problems are detected by evaluating the monitored signals of the sensors attached to the structure. Active SHM systems are similar to non-destructive evaluation (NDE) methods. Both categories of techniques are trying to detect the presence and extent of damage in the structure. The only difference is that the advances in sensors and embedded electronic systems have made it possible to have them be permanently installed on the structure and provide online information about its status. However, the size and cost of conventional NDE transducers impedes them being used directly installed on structure for SHM purposes. Piezoelectric elements and recently developed miniature actuators/sensors allowed the implementation of SHM methods in many applications. Structural health monitoring using piezoelectric elements is performed via multiple approaches [2]: (a) wave propagation (b) frequency response function (c) electromechanical impedance.

\subsection{Rayleigh-Lamb Surface Guided Waves}

Guided waves are elastic waves that propagate in solid structures. Lamb waves are a type of guided wave that propagates in plate and shell-like structures with free surfaces. The mathematical analysis of Lamb waves was developed and published by $\mathrm{H}$. Lamb in 1917 [7]. Based on the pattern of motions, Lamb waves could be divided into two categories of symmetric and anti-symmetric waves (Figure 1-1). 
(a)

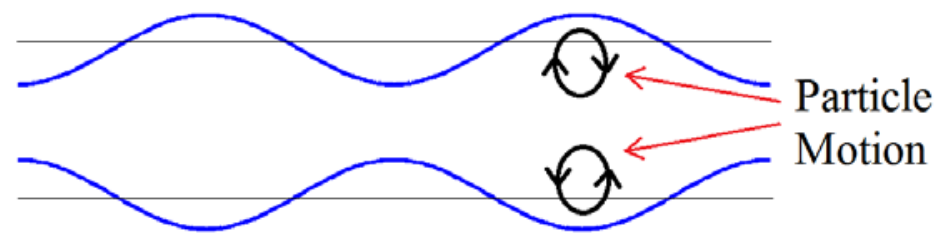

Symmetric

(b)

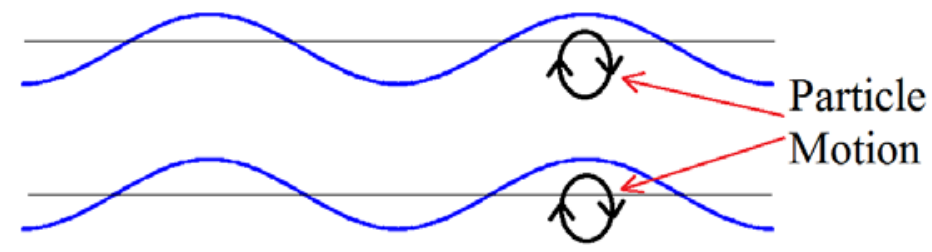

Anti-symmetric

Figure 1-1 Lamb wave modes (a) Symmetric (b) Anti-symmetric

Rayleigh waves are another type of guided wave that travels close to the surface of solid structures [8]. They include both longitudinal and transverse motions and could be generated by piezoelectric-based transducers. Guided waves particle motion happens on a single surface. Worlton produced guided waves for the first time experimentally in 1961 [9] and realized their potential application in non-destructive tests. Mindlin, in 1951 [10], completed a comprehensive theory for elastic waves and the governing equation of motion was presented in the form of:

Equation 1-1: $\quad(\lambda+\mu) \nabla \nabla \cdot \boldsymbol{u}+\mu \nabla^{2} \boldsymbol{u}=\rho \ddot{\boldsymbol{u}}$

Where $\boldsymbol{u}$ is the displacement vector along the 3-axis coordinate system. $\lambda$ and $\mu$ are Lame's constants for the isotropic material while $\rho$ is the density. Mindlin used the Helmholtz decomposition method to split the equation into scalar and vector components. Then, considering the directional propagation of the waves, he found the harmonic solution for the set of differential equations. Applying the traction-free boundary 
conditions, and the zero determinate condition for the non-trivial solution of matrix equations led to the Rayleigh-Lamb equations:

Equation 1-2: $\quad \frac{\tan \beta b}{\tan \alpha b}=\left[\frac{-4 \alpha \beta \xi^{2}}{\left(\xi^{2}-\beta^{2}\right)}\right]^{ \pm 1}$

Here, $\mathrm{b}$ is the thickness of the plate, and $\alpha$ and $\beta$ are constants based on the bulk longitudinal and shear wave speeds, respectively.

These equations yield the relation between the excitation angular frequency $\omega$ and the phase velocity $c_{p}=\omega / \xi$ which is called dispersion property. This means that the wave velocity is dependent on the frequency of the wave. This phenomenon could be presented by dispersion curves [Figure 1-2].

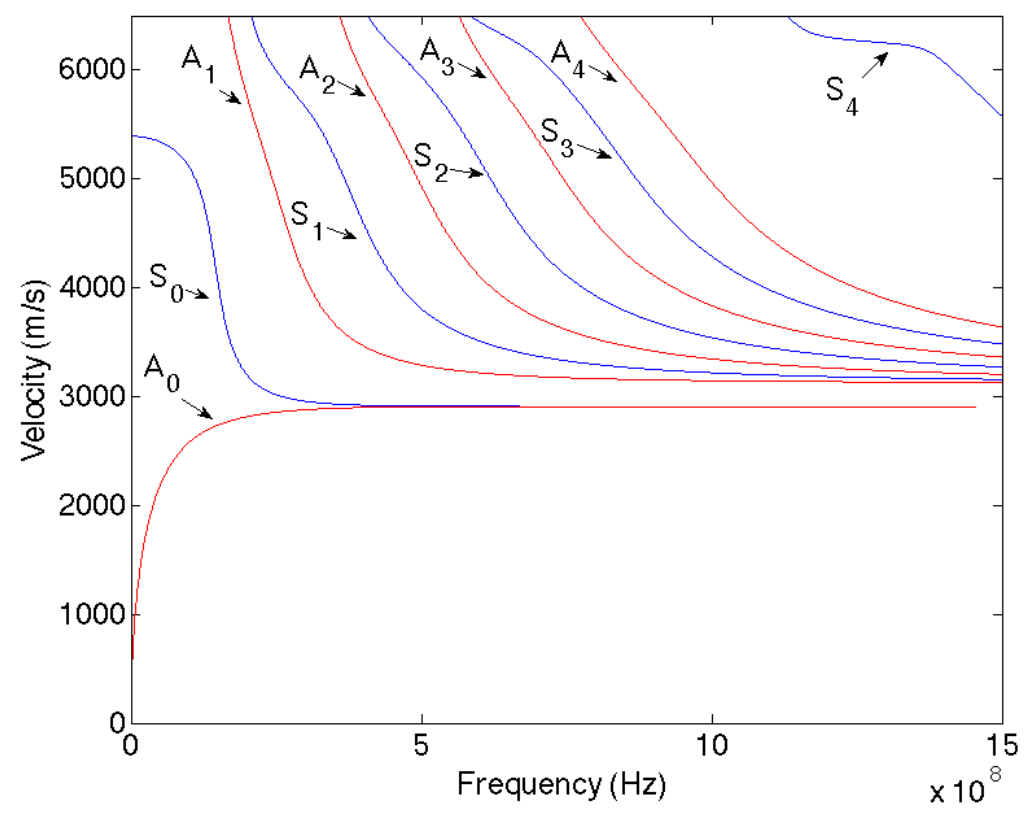

Figure 1-2 Dispersion curves for symmetric and anti-symmetric lamb waves [1] Surface-guided waves are employed in structural health monitoring due to their unique property of being able to travel long distances with minimal dissipation. This allows for monitoring a large area on the target structure from a single sensor point while 
limiting the number for sensors required to establish the SHM sensor network. In the lamb-wave-based damage detection techniques, knowing the propagation velocity of the certain frequency of guided wave, it is possible to detect the surface damages by monitoring the delay from a returning pulse [6].

\subsection{Electromechanical Impedance Method}

The Electromechanical Impedance (EMI) method is a new emerging structural health monitoring method. It has been proven that the presence of damages and structural defects causes the mechanical impedance of structure to change. However, it is very difficult to directly measure the mechanical impedance of structures with different shapes and configurations. On the other hand, piezoelectric transducers are used to indirectly measure this quantity $[5,62,108]$. Once a piezoelectric transducer is bonded to the mechanical structure, an electromechanical coupling exists between the electrical impedance of the piezoelectric transducer and the mechanical impedance of the structure. The electromechanical admittance Y depends on mechanical impedances of monitored structure $Z_{M}$ and the piezoelectric transducer $Z_{a}$ according to work by Sun et al. [108]:

Equation 1-3: $\quad Y=j \omega a\left[\varepsilon_{33}^{T}-\frac{Z_{M}}{Z_{M}+Z_{a}} d_{31}^{2} Y_{11}^{E}\right]$

The superscripts $\mathrm{E}$ and $\mathrm{T}$ stand for mechanical stress and electric field. $Y_{11}^{E}$ is Young's modulus, $\varepsilon$ is electric permittivity and $a$ is geometric constant of the piezoelectric transducer.

Park and Inman [112] determined the electromechanical impedance by directly measuring the voltage and current from the PZT element (Figure 1-3). 


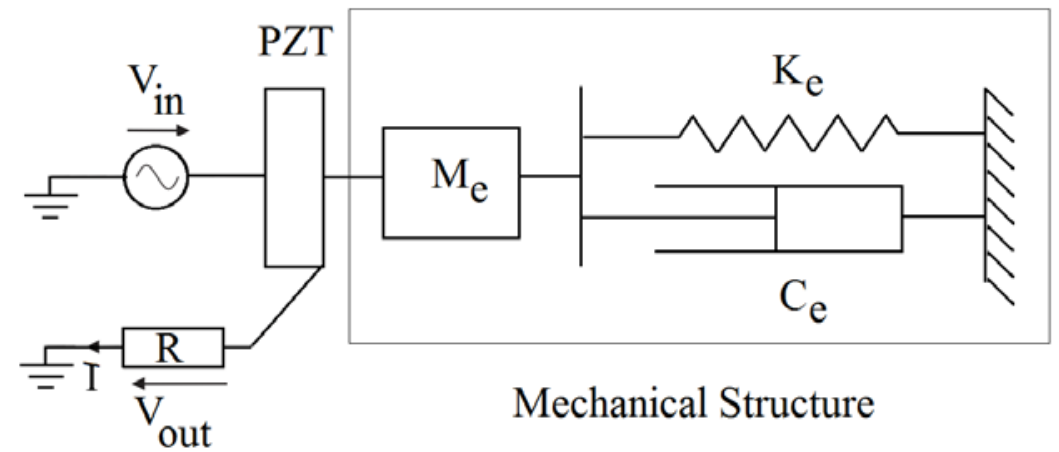

Figure 1-3 Schematic of indirect measurement of mechanical impedance of structure using electromechanical impedance (EMI) method

In SHM of aerospace structures, promising results has been obtained in detecting damages by the EMI method in the vicinity of piezoelectric transducers. However, for failures distant from the piezoelectric transducer, the method showed to be insensitive. In fact, the effective range is in millimeters and centimeters rather than meters [109]. The introducing damage to structures causes the impedance diagram to change, which is manifested by frequency shifts in some peaks, emerging of new peaks and increase of some peaks’ amplitudes [1].

A key issue in the correct estimating the size of failure in the EMI method is the proper selection of frequency range [110]. Various types of flaws including fatigue cracks and corrosion in aluminum riveted panels [111], delamination in composite patches [110] and loosenings in piping bolt joints [112] can be identified using this method. The majority of literature results have been obtained using laboratory the impedance analyzer, which is considerably expensive and bulky, and the practical implementing the method is pending on further hardware and software developments [27]. For measuring the impedance of the host structure using the EMI method, both direct piezoelectric effect (Figure 1-4) and inverse piezoelectric effect are used. 


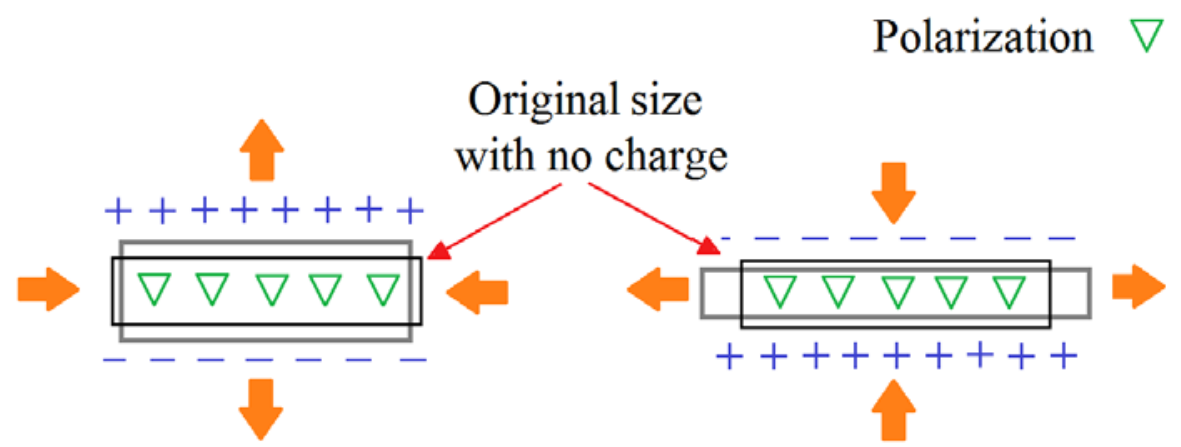

Figure 1-4 The contraction and expansion of PZT material due to direct piezoelectric effect

First, the structure is excited based on the inverse piezoelectric effect, where the strain is produced in the transducer due to electrical charge and the strain is transferred to the structure due to the bonding. Then, the electrical impedance is quantified based on the direct piezoelectric effect, where electrical charge is induced in piezoelectric elements due to the presence of strain. The electromechanical impedance is determined by directly evaluating the voltage and current from PZT [112].

Analytical models for simulating the electromechanical impedance method are limited to simple structures like plate, beam and rod $[37,38]$. Numerical modeling of the EMI method has been subjected to previous studies in order to predict the crack growth or find the location of PZT transducers on the surface of the structure. Coupled field analysis, which takes both mechanical motions and electrical characteristics into account, was found to be the most efficient method for modeling complex structures $[39,40]$.

The surface response to the excitation method (SuRE) [29] monitors the health of the structure using characteristics similar to the EMI method. A piezoelectric element excites the surface waves and another piezoelectric element monitors the surface waves in another point on the structure. Piezoelectric elements are inexpensive and their 
sensitivities are very high. However, it is not practical to attach tens of piezoelectric elements to the surface to evaluate the surface response characteristics of many points. In this study, the scanning laser vibrometer (SLV) was used to evaluate the surface response characteristics at a grid of scan points. The SLV uses the Doppler effect to measure the surface oscillations and its sensing mechanism is illustrated in Figure 1-5.

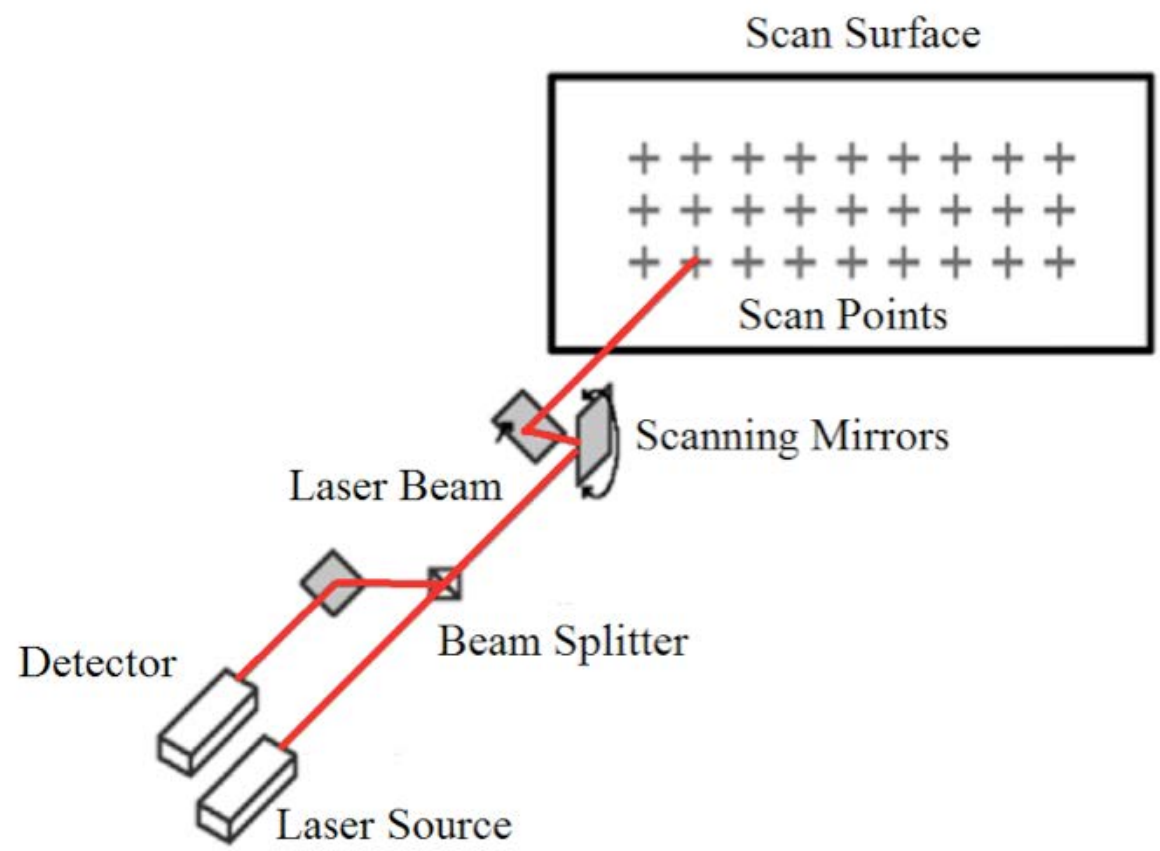

Figure 1-5 scanning laser vibrometer structure and sensing mechanism

The method has proved to be effective in detecting a variety of structural defects, including tool wear [31], loose bolts [32] and compressive loads in beam [33] and plate [34] structures. The SuRE method requires a separate sensor in addition to the piezoelectric actuator. This technique, which has been used for the experimentations of this study, will be further discussed in chapter 3 . 


\subsection{Manufacturing Process Monitoring}

For many years, monitoring technology was limited to expensive aerospace industries. Recently, due to advances in computer systems, sensor technology and data processing, it is possible to develop specialized monitoring systems for other fields, including automated manufacturing. In classical tool condition monitoring (TCM) [35], several parameters like cutting force, vibration and temperature have been used to monitor the tool life (Figure 1-6).
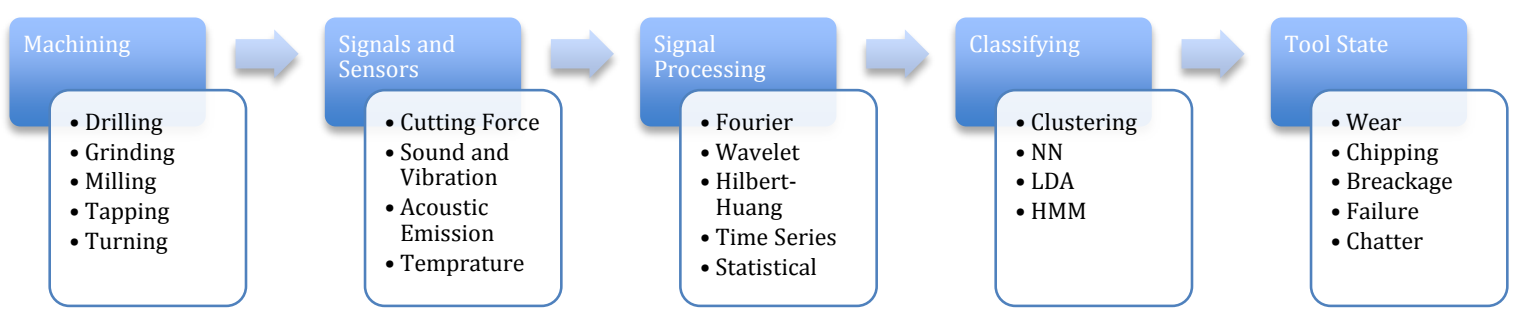

Figure 1-6 The framework of tool condition monitoring [114]

The new trend in manufacturing monitoring is the machining process monitoring (MPM), where the goal is to monitor the entire machining process to increase the quality and accuracy of the final product while reducing costs originating from tool failure and machine shut-down time [114]. MPM is needed not only to increase the productivity and product quality, but also to identify the risks to damage of the work-piece and machine components.

Manufacturing society has been using methods similar to SHM techniques for process monitoring. Recently, part-based manufacturing process performance monitoring (PbPPM) was introduced to monitor the manufacturing process based on the data collected directly from work pieces [36]. The purpose of this method is to detect various 
manufacturing malfunctions, including tool breakage, chatter, manipulator out of calibration, and surface roughness by only monitoring the work-piece.

\section{Part based Process Performance Monitoring (PbPPM)}

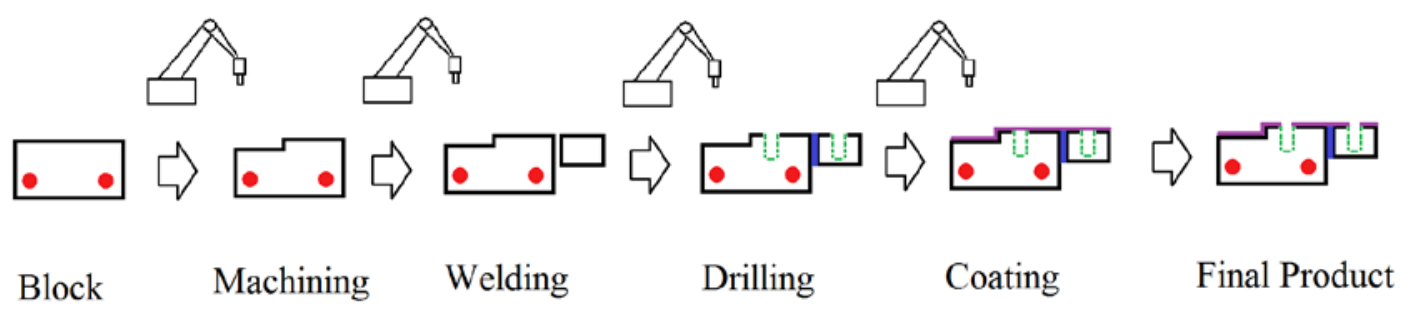

Figure 1-7 part-based process performance monitoring (PbPPM)[36]

\subsection{Organization of the thesis}

This dissertation consists of eight chapters. At the beginning, we will illustrate the potential of the surface response to the excitation method for SHM and MPM by using the finite element method. Then, the SuRE method, will be implemented using the scanning laser vibrometer to improve its capabilities. Later, this method will be used to monitor the machining applications. The performance of a digital signal processing-based device developed for implementing the SuRE was compared to laboratory equipment results.

- In chapter 1 , an introduction to the structural health monitoring and the contribution of techniques of this study is presented.

- In chapter 2, the state of art in structural health monitoring (SHM) and manufacturing process monitoring (MPM) is presented. 
- In chapter 3, the surface response to the excitation method is introduced and signals, computational methods, signal processing, experimental set-up and implementation techniques are discussed.

- In chapter 4, COMSOL is used to simulate the surface response to excitation method in a frequency domain study and evaluate its potential application for manufacturing process monitoring.

- In chapter 5, the loading condition detection capability of the SuRE method is assessed using the scanning laser vibrometer and then applied to examine the integrity of loose bolts.

- In chapter 6, the SuRE method is evaluated for remote performance monitoring of manufacturing machining operations, including drilling, cutting and milling.

- In chapter 7 , the performance of the SuRE method, the reliability of each implementation technique and the computational accuracy of its algorithms are compared to each other.

- In chapter 8, the pros and cons of the SuRE method for SHM and Part-Based MPM are presented and the potential for future work is discussed. 


\section{REVIEW OF PREVIOUS WORKS}

In this chapter, a literature survey over the state of the art of structural health monitoring using high-frequency surface guided waves is presented. An overview of the most impactful and recent studies for numerical simulations, measurement techniques, damage detection algorithms, signal processing methods, decision making strategies and machining monitoring systems is covered.

\subsection{Major SHM methods for aerospace applications}

The propagation of elastic surface guided waves on the structures has been studied extensively and structural health monitoring (SHM) systems have been developed [41]. Guided Lamb-wave-based methods [42,41] and electromechanical impedance (EMI)-based methods [58,59] are the most commonly used active SHM methods.

The surface guided waves (Lamb waves) are introduced to the structure through a piezoelectric actuator in the Lamb wave approach and the propagation of them is monitored at the same and/or additional point(s) via sensor(s). Structural problems are detected from the change of the characteristics of the monitored signals. Generally, development of additional wave patterns and their delays indicated the presence of defects and their locations. A comprehensive state of art for these methods is presented at reference [42]. This approach can be used for many large structures, since the generated Lamb waves are capable of traveling along the plates of many materials with very little attenuation [43, 44, 45]. However, the difficulty of sampling such a high speed wave, following multiple wave modes, complexity of wave propagation and reflections from boundaries creates significant challenges for the researchers [46]. 
The second major SHM approach is the impedance method [5]. This approach evaluates the impedance of a piezoelectric element attached to the surface of the structure. Experimental studies have shown that the impedance characteristics within a carefully selected frequency range change, when various defects are created at the structure or loading changes. Most of the researchers used the impedance analyzer, which automatically generates the signal and analyzes the collected data.

Tansel et al. [47] developed the surface response to excitation method (SuRE) to evaluate the same characteristics by using a simpler experimental setup. The SuRE method evaluated the surface-response characteristics similar to the EMI, but required simpler instrumentation. A piezoelectric element excites the surface and the surface response is evaluated with a similar piezoelectric sensor. The EMI and SuRE methods calculate the sum of the squares of the differences of the real part of the impedance and magnitude of the transfer function, respectively. The impedance method is more sensitive to the loading conditions compared to the Lamb wave method. In the impedance method, only a single piezoelectric element is used both for exciting and sensing. Detecting a point load on the structure could be used for detecting the loose bolts in the joints. This way, the integrity of structural bolt joints could be examined using this method and any possible loose components could be identified.

Most of the researchers have used the laser vibrometers for health monitoring by evaluating the modal parameters like mode shapes, natural frequencies and damping ratios [1-48]. Sharma [49] used the scanning laser vibrometer to get information to calculate the strain energy distribution for defect detection. Staszewski et al [50-51-52] have used the laser vibrometer to capture the Lamb waves to evaluate the integrity of an 
aluminum plate. Marterelli [53] also made important contribution to laser-based scanning. Leong et al. 52] used SLV in an effort to detect fatigue cracks using highfrequency guided waves. In their study, the Lamb wave method was used.

Since the impedance method uses only a single piezoelectric element, it is not possible to implement this technique using non-contact SLV sensors. However, separating the sensor and exciter in the SuRE allows the SLV to collect data from a virtually infinite number of points. This way, the SuRE will have both advantages of the Lamb and the impedance method. Like the Lamb wave method, The SuRE method will cover large areas since it uses surface guided waves and at the same time it will be able to detect the loads since it has similar characteristics to the impedance method. This will be discussed further in chapter 5.

\subsection{Inspecting bolts by using SHM methods}

Many structural health-monitoring (SHM) techniques are developed to improve the reliability and safety of the systems, while the maintenance costs and service time are reduced. Some of these methods are developed for detecting loose bolts. Bolt joints have been widely used in many civil, mechanical and aerospace structures. In some critical operations, any failure could have catastrophic consequences and manual inspection of the bolts is not feasible. The development of remote monitoring techniques is necessary to address these applications.

Todd et al. [57] evaluated the condition of bolt joints by using the modal parameters. He experimentally found that the modal properties were relatively insensitive to the clamping force as long as they were above a critical level. Todd suggested 
evaluating the modal parameters might not be a reliable approach for assessing the condition of the bolt joints. He recommended alternative methods should be considered for developing more sensitive monitoring techniques.

Peairs et al. [13] employed the impedance-based structural health monitoring technique by applying high-frequency excitations through a PZT transducer. He found that the impedance characteristics were sensitive to loosening bolts. Peairs also tried to replace the impedance analyzer with a smaller and more effective device, since it was heavy and bulky. Ritdumrongkul et al. [60] studied the structural integrity of two aluminum beams connected through a bolted joint. They found the EMI method promising; however, it was mentioned that the application of the method to real structures required further study of the characteristics of more complex structures and environmental effects. Chakraborty et al. [61] introduced an advanced time-frequency signal processing technique for detecting loose bolts in complex structures. The method required a significant amount of experimental data for the training of the damage classification algorithm. Chakraborty suggested the use of numerical simulation for preparing training data.

Previous studies have proven that the impedance method performed considerably better for health monitoring of bolted joints like gas pipelines and composite structures [29,62]. The location of the loose bolts could be estimated if multiple elements were installed carefully. In critical applications like the integrity of bolt joints in satellites [63], it was hard to access and impractical to create a large network of PZT sensors.

Liang et al. [63] first developed a one-degree of freedom model for a coupled electromechanical PZT actuator system. The study showed that the change of the 
structural impedance would dominate the electrical impedance of the piezoelectric element. Monitoring the impedance of the PZT with an impedance analyzer was enough to determine the condition of the structure. To evaluate the condition of the structure, the real part of the impedance was analyzed, since it was more sensitive to structural damages [64].

In section 5.2 of chapter 5 , the integrity of a bolted-joint section of a robot will be inspected by non-contact implementing the surface response to the excitation (SuRE) method using the SLV. The motivation behind this study is to introduce SLV as an alternative to sensor networks. In many critical applications, such as the inspection of the control surfaces of rockets at the launch pad, the use of SLV could be easily justified. The frequency domain methods have not been implemented through non-contact sensors for health assessment of multi-bolt joints in previous studies.

\subsection{Detection of structural defects with SHM methods}

All guided-wave-based structural health-monitoring methods use some sort of signal processing for sensory data. The objective is to extract some information about the presence and location of damage, type of damage and its severity for prognostics of remaining life. In the Lamb-wave-based methods, the damage is detected by calculating the time delay of the incoming pulse. An effective signal processing method isolates the time-frequency centers of the pulses from propagated waves, specifies their associated modes and addresses the issue of noise in the signal [41]. Although it is possible to borrow some of the techniques from non-destructive evaluation (NDE), the algorithms of SHM must be computationally efficient enough to run real-time. 
Preprocessing and data cleansing is necessary, especially where the sensor is susceptible to noise or the feature extraction is not robust to noise. These groups of techniques include statistical averaging [117], wavelet transform and filtering [118], and outlier reduction [119]. A comprehensive review of low-pass filters for data cleansing is presented in the reference [120].

After data cleansing, the proper features and parameters for damage detection are selected and extracted from the signal. Features are the best parameters of signal to represent the health condition of the structure. The Lamb guided wave-based methods are generally categorized into time-frequency analysis and sensor array approaches. In fact, the features are inputs for any pattern recognition algorithm [121]

While Fourier analysis only reveals the frequency content of the signal, it does not provide any information about when each of the components arrived to the sensor. In the time-frequency representations (TFRs), the diagram involves a time axis that actually shows when each of the frequency components entered the signal. These techniques are well suited to Lamb wave methods that work based on sending-receiving pulses and figuring out the damage by calculating the time delay. This is important, especially considering the dispersive property of Lamb-waves where different frequency and mode shapes of guided waves travel with different speeds. Once the time-frequency image is generated, the locations of frequency centers are identified to find the exact time interval (Figure 2-1). 

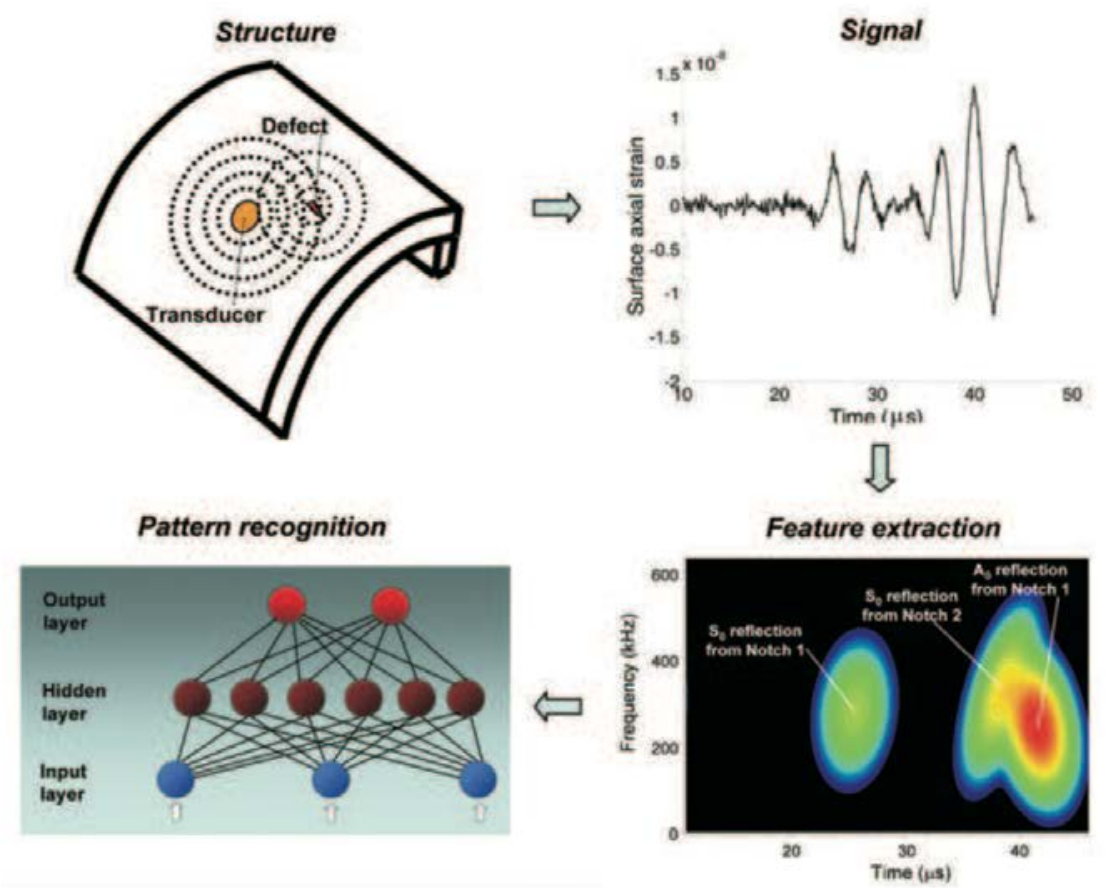

Figure 2-1 Essential steps in guided-wave-based structural health monitoring [41]

Various techniques [122] have been used to isolate multiple closely space guided wave modes in time and frequency. Recently, wavelet transform has emerged as a very important signal processing technique for feature extraction and the selection of signals [123]. There are two types of wavelet transforms; the continuous wavelet transform is more suitable for time-frequency graph generation while the discrete wavelet transform is mostly suitable for feature extraction and selection.

The second major trend in Lamb-guided wave-based techniques is using an array of sensors on the structure. This allows both time and spatial dimensions to be considered in calculations. The goal is to identify the individual guided wave modes and their corresponding amplitudes at certain propagation distances [124]. Since it has been proven that different modes of guided waves are sensitive to different types of defects, an array of sensors could target various types of structural damages [125]. Some algorithms use a 
linear sensor array for directional detection [126]. The idea is to use an appropriate signal processing to find the direction of incoming waves and therefore virtually scan the structure without moving the sensors. Another advantage of this approach is that a radar type of scanning sensors allows keeping the number of sensors minimal [127]. Beamforming [128] is a signal processing technique that is developed based on the linear array of piezo sensors for damage detection and localization. Methods based on beamforming have been able to detect cracks that are not directly located in the field of sensor arrays. Methods based on this technique have been able to locate the damage with exceptional accuracy in isotropic plate structures [129].

Integrated solutions are efficient designs of guided-wave-based SHM systems that include sensors and actuators, wirings and data processing within an embedded system. Among theses technologies are: "SMART Suitcase" [134], Integrated Vehicle Health Management "IVHM" [135] and the diagnostic network patch [136]. Some of the crucial issues involved in development of integrated systems are to include wireless communication capability to address the issues related to wires and local computational power to limit the volume of data required to be transferred. In the case of active SHM techniques, the power supply for actuators is another challenge in front of the embedded solution. (Figure 2-2) 


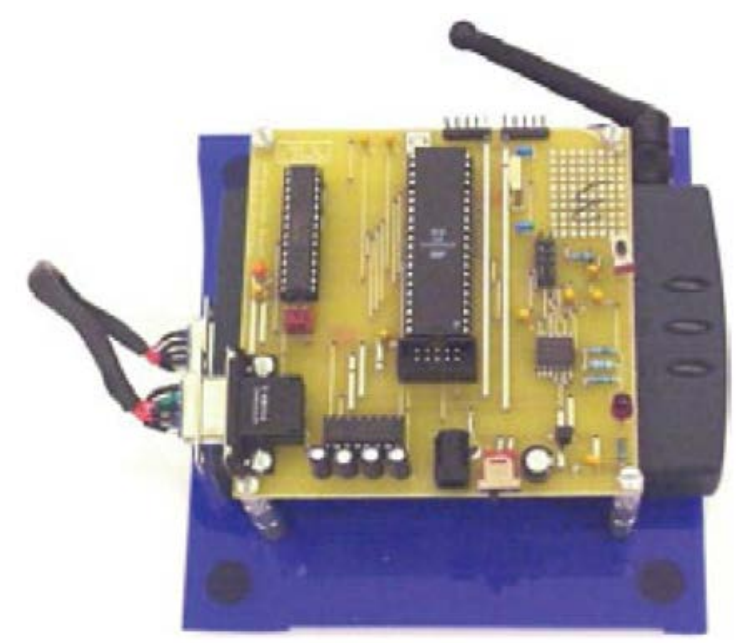

Figure 2-2 Wireless embedded device developed by Lynch et al. [137]

2.4. Automated interpreting SHM signals with neural networks

Pattern is a certain behavior of selected features that could be used to indicate the state of health of structure. Neural networks are one of the most popular pattern recognition techniques used in guided wave-based damage detection techniques in plates. Spectrographic features in the time-frequency domain have been used to train neural networks. Practical application has been proved in composite laminates [130], aluminum T-joints [131] and metallic welds [132]. To overcome the problem of overtraining, the support vector machine (SVM) was used to classify defects in metal matrix composites [133].

Neural networks are systems composed of simple elements operating in parallel. These are inspired in biological nervous systems. The network function is determined by the connections between the elements (nodes). The training of the neural network consists of adjusting the values of the connections between elements (weights) [72]. For computing the weights of these elements several types of algorithms can be used. These 
algorithms differ in the activation and training function used for connect nodes of the neural network

The Levenberg-Marquardt algorithm [73] was designed to approach second-order training speed without having to compute the Hessian matrix. When the performance function has the form of a sum of squares (as is typical in training feed forward networks), then the Hessian matrix can be approximated in terms of the Jacobean. For a more detailed discussion of the scaled conjugate gradient algorithm see Ref [74]

The scaled conjugate gradient algorithm is based on conjugate directions, but this algorithm does not perform a line search for each iteration. For a more detailed discussion of the scaled conjugate gradient algorithm see Ref

In order to compare which algorithm has a better performance, the following function to measure the error is used:

Equation 2-1 $\quad f_{\text {error }}(\mathrm{Z}, \tilde{Z})=100 \times \frac{\sum_{i=0}^{n}\left|Z_{i}-\widetilde{z}_{l}\right|}{(\max (Z)-\min (Z)) \times n}$

where, $\mathrm{Z}$ is target function vector, $\tilde{Z}$ is neural network output vector and $\mathrm{n}$ is number of points of the matrix of reference.

\subsection{Monitoring the performance of machining operations}

Machining process monitoring is an essential part of the automated manufacturing processes $[75,76]$. The purpose is to improve tool maintenance and product quality. The need for manufacturing automation and demand for more sophisticated parts are major reasons for the development of intelligent machine tools. There are still major challenges for the practical application of current monitoring systems due to the reliability and costly 
implementation procedure. Many methods have been developed to evaluate the manufacturing operations; however, the complexity and the cost of these systems limit their implementation in industry.

There are two fundamental approaches in the machining condition monitoring field, direct and indirect methods [77,78]. Direct methods such as vision-based or optical methods directly measure the dimensions of the tool or the work-piece to identify the product quality. Their application requires the tool and work-piece to be cleaned from the chips and fluids.

During the metal cutting operation, chips and cooling fluid could easily block the vision of the camera. Due to this reason, although being extremely accurate, the practical application of direct methods has been limited to the laboratory. On the other hand, the indirect methods have the advantage of not interrupting the operation. During the manufacturing operations, several parameters influenced and could be employed for monitoring the state of the tool or metal removal process [79]. Among those parameters measurement of cutting forces [80,81], acoustic emission signal [82,83], ultrasonic signal [84], currents of servo drivers [84] and the combination of them [86] have been used more frequently.

Various signal processing methods could be used to process the data. Wavelet Transform [87,88], Short Fourier Transform [6], Hilbert-Huang Transform [89] and singular value decomposition method [90] were used as processing techniques for the above-mentioned signal readings. Also, in multi-sensor methods, a combination of the sensor data has been used [91] and its sensitivity was compared [92]. The last step in developing a process monitoring technique is to find a decision-making approach. When 
developing any machining process monitoring method, the amount of complexity of the system should be considered carefully. Otherwise, the complexity of the system would compromise any future industrial application.

Hsieh et al. [98] applied a neural network method for spindle vibration-based tool wear monitoring in micro- milling. However, their methodology was not able to distinguish if the change in energy level of the other frequency domain signals was due to the worn tool or they were affected by changes in parameters such as material and noise. Cus et al. [99] used the Adaptive Neuro-Fuzzy Interference System (ANFIS) to predict the flank wear of the tool in the end milling process. Neural network was used as a decision-making system to predict the condition of the tool and the cutting forces are used as an indicator of the tool flank wears variation. However, due to the high computational power required for training the neural network, their method required parallel processing for the monitoring of the cutting process with high reliability; they suggested different decision-making tools, such as fuzzy logic, to be applied to obtain a smaller error of detection.

Brecher et al. [103] used NC kernel data for surface roughness monitoring in milling operations. He used experimentation in order to obtain the data to be modeled with artificial neural networks (ANNs) for surface roughness average parameter predictions. The major drawback of the method was that it required performing several prior cutting tests in the corresponding machine tool, cutting tool, tool holder, and material combination. Huang [104] developed an intelligent neural-fuzzy model for an inprocess surface roughness monitoring system in end milling operations. The disadvantage of fuzzy-net was that when the number of fuzzy sets was increased, there was a need for 
training data to be increased to fulfill all the possible IF-THEN rules. Marinescun. et al. [105] developed an on-line automated monitoring method based on acoustic emission (AE) measurement for surface anomalies during the milling of aerospace alloys. Their method reduced surface anomalies by a process monitoring solution that detects work piece anomalies associated with the cutting tooth of a milling cutter through signal analysis, which causes improvement of fault detection and the avoidance of surface anomalies or tool malfunctions. Although the method was efficient in controlling the tool to minimize process malfunctions in milling operations, it was reported that in higher feeds and speeds, the system was limited by the processing capability of the operating system and amount of data processing needed to detect the malfunctions. Quintana et al. [106] developed a process solution that controls the surface process based on artificial neural network models by capturing the vibrations that occur during metal removal operation with the help of piezoelectric accelerometers. The method calculates the current in-process roughness average, looks at the cutting parameters and applies the neural network developed using dynamic parameters measurement. Like similar methods, an extensive number of experiments is required to be carried out to increase the software performance by training the network on the basis of a large number of experiences. Bisu et al. [107] proposed a method based on the vibration analysis that refers to the spectral envelope based on Hilbert transform, and identifies mechanical defects to obtain a better response on the milling process quality. In chapter 6 of this study, the SuRE method is used to develop an efficient non-contact milling process monitoring. 


\section{SURFACE RESPONSE TO EXCITATION (SURE) METHOD}

\subsection{Introduction}

The Electromechanical Impedance Method (EMI) monitors the impedance change of a piezoelectric element as it reacts to vibrations in the structure. The Lamb Wave Method monitors the propagations of the waves. It is used to estimate the location of the defects by measuring the delay of the arriving wave; however, it is not as sensitive to the variation of the compressive forces applied to the structure as the Impedance Method. In practice, most of the researchers use an impedance analyzer such as HP 4194A for characterization of the piezoelectric element when implementing the Impedance Method. These impedance analyzers are expensive, their excitation signal power is limited, and the user has limited control over determining the transfer function or excitation signal. They may also have problems when the piezoelectric elements are attached to long wires or there are additional components in the circuit. Analog Devices like 5933/5934 impedance-to-digital converter (IDC) offer another approach to measure impedance; however, IDCs are not as powerful as impedance analyzers. When the impedance analyzers and IDCs are used, the user selects the test frequencies. The device manages signal generation, data collection and analysis. There are two approaches alternative to impedance analyzers. The first approach directly replaces the impedance analyzer with a circuit $[12,13,14,15]$. A data acquisition system with a programmed real-time analyzer, Digital Signal Processor (DSP) or spectrum analyzer may be used to monitor the characteristics of the piezoelectric element with the help of a relatively simple circuit. The second approach excites a piezoelectric element attached to the structure. The dynamic characteristics of that piezoelectric element and the attached structure are 
monitored using second piezoelectric element $[16,17,18]$. The spectrum analyzer or signal generator-voltmeter combination $[17,18]$ have been used for this approach. Tansel and co-workers called this approach Surface Response to Excitation (SuRE) [30] .

\subsection{Excitation and sensing of the SuRE method}

The Surface Response to Excitation (SuRE) method is an emerging structural health monitoring technique. With similar characteristics to the electromechanical impedance method, the state of health of the structure is determined based on changes in signature characteristic spectrums. The difference is that the signature spectrum is generated as the frequency transfer function between the excitation point and the sensing point. Therefore, in the SuRE method, the excitation and sensing are performed using separate sets of elements. This means that instead of only one piezoelectric transducer making measurement from a single point, the measurements could be captured from any point on the structure.

The SuRE method excites the high-frequency surface-guided waves over a range of high frequencies. The excitation is in fact in the form of sine wave covering from starting frequency to stopping frequency. Usually, the minimum start frequency is 20 $\mathrm{kHz}$, slightly above the audible zone to avoid the hazardous sound in the lab environment. The maximum of the end frequency depends on the maximum speed of analogue to digital conversion available in the data acquisition card. Another parameter that limits the maximum end frequency is the exciter transducer's specification. In the experimentations of this study, this parameter varies depending on the equipment or device being used. A schematic of the SuRE method is shown in Figure 3-1. 


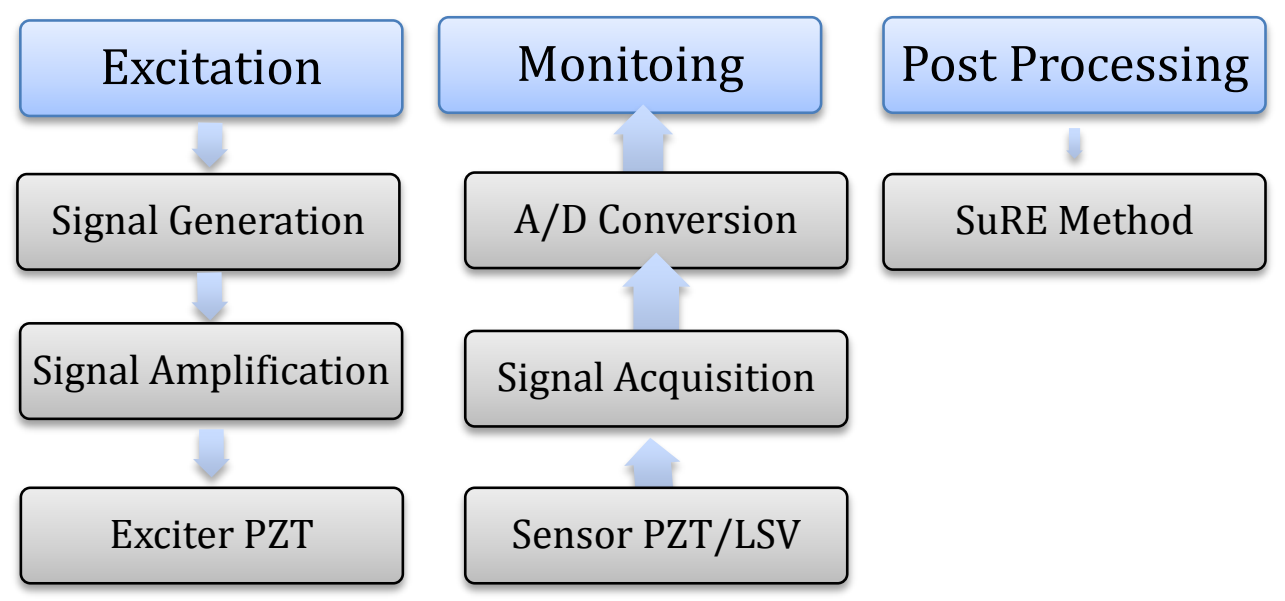

Figure 3-1 Schematic of the Surface Response to Excitation (SuRE) method

The SuRE method has advantages from both Lamb-wave-based and EMI-based approaches that were described in the previous sections. Similar to Lamb-wave-based techniques, the surface guided waves are being excited in the SuRE method and it is possible to monitor large areas on the structure, and the area of detection is not limited to the vicinity of the transducer. On the other side, using the signature spectrum for SHM allows the SuRE method to retain some of the unique characteristics of the EMI method. The fundamental assumption of the SuRE and the impedance method are similar. The impedance method assumes that the real part of the impedance of any point on the structure within a carefully selected frequency range is like a fingerprint. For the SuRE method, this is the magnitude of the transfer function or the spectrum of the monitored signal.

This representative curve should be the same as long as the structural integrity or loading is not changed. The spectrum of the monitored signal without any change at the 
loading condition is presented in Figure 3-2 (a). The spectrums changed drastically when the load was applied in Figure 3-2 (b). [33]

(a)

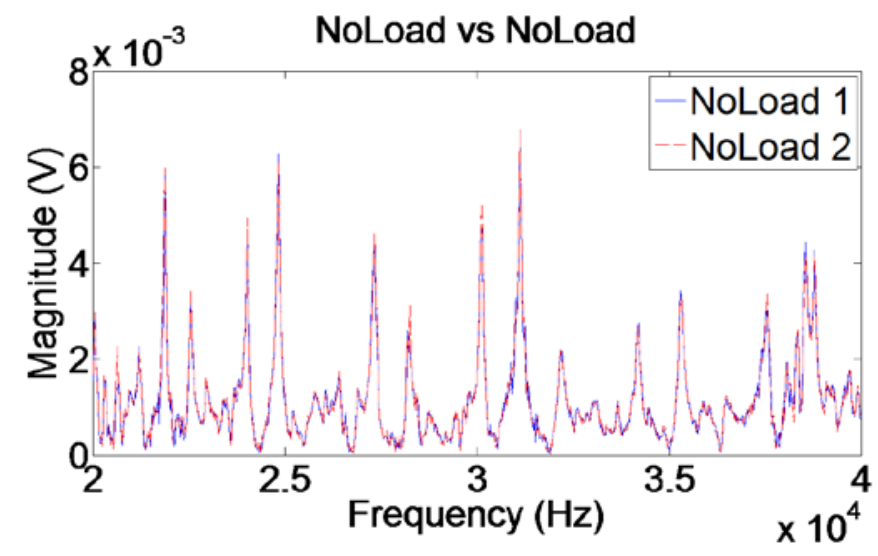

(b)

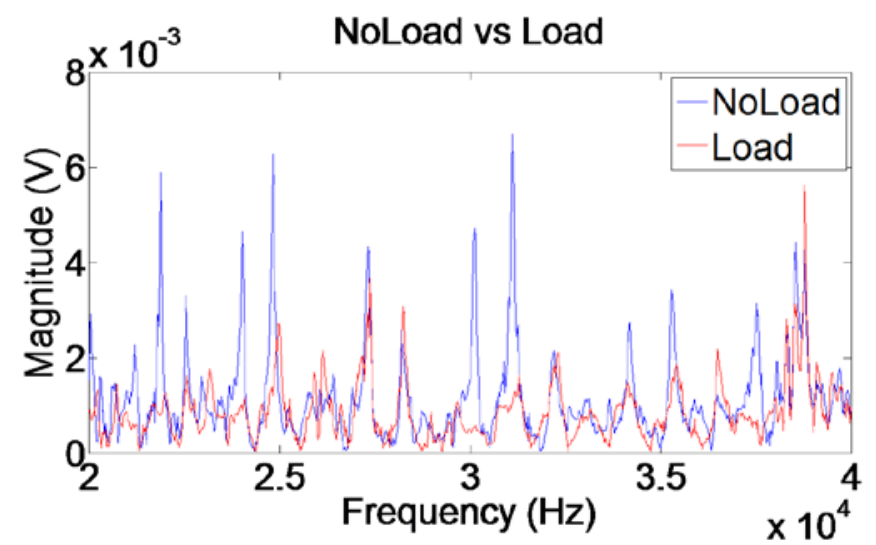

Figure 3-2 (a) The spectrums of a point on the grid for two successive no load scans (b) Spectrums of a point on the grid before and after the load was applied

\subsection{Excitation signal and analysis of the sensory data}

There are two major excitation and signal analysis approaches for the SuRE method. The first method excites the surface with a since wave and analyzes the sensory signal by using the Fast Fourier Transformation (FFT). The spectrum is captured by the peak hold of simultaneous FFT of the incoming signal from the sensor.

Figure 3-3 shows capturing the peak of FFT of the measured signal during a complete sweeping cycle created the frequency spectrum. Since the excitation frequency at any time is a pure sine wave, in the frequency domain it has only a single peak. Since each 
excited frequency transfers with different amplitudes, as the sweep happens, the amplitude of peak drops and increases. The peak-hold procedure draws the signature spectrum by capturing the footprint of the sweep at each frequency as it passes throughout the entire frequency range.

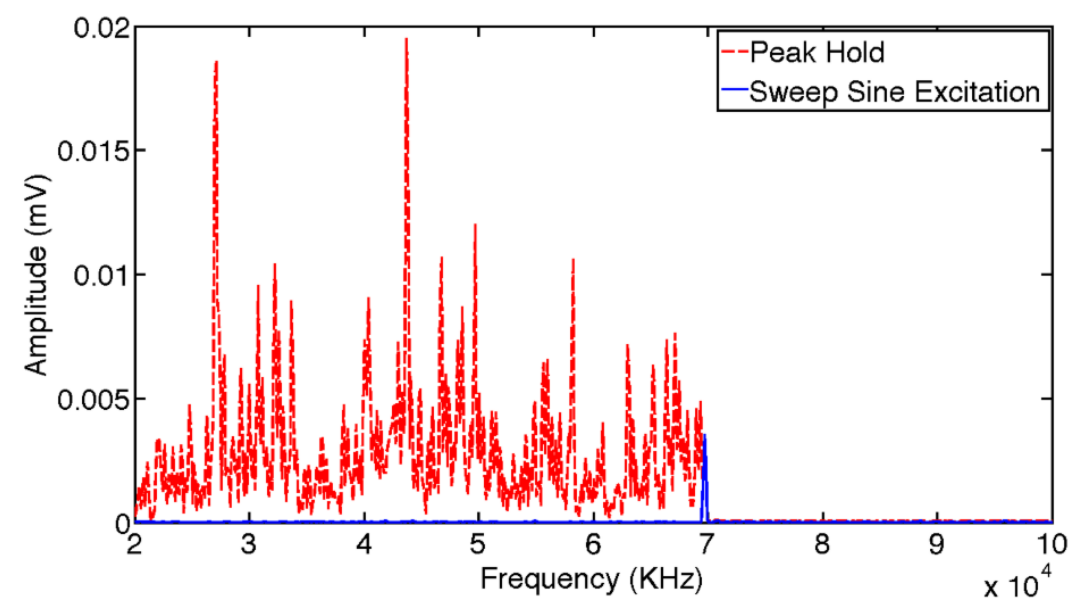

Figure 3-3 FFT of sweep sine wave excitation and corresponding peak hold spectrum

In the second approach, the time domain data is used directly to generate the frequency transfer function. For this purpose, the structure is excited at a series of frequencies one by one. In this study, the Teager-Kaiser (TKA) [19,20], Goertzel [21], RMS and the average of the positive readings algorithms will be discussed for estimating the characteristics of the piezo-structure combination.

The advantage of the second approach is that it allows embedded implementing of the SuRE method using digital signal processors (DSPs). While complicated signal transforms are hard to implement on embedded system, simpler amplitude estimation algorithms could be efficiently used to capture the system characteristics. An example of a typical time domain set of data is shown in Figure 3-4. 

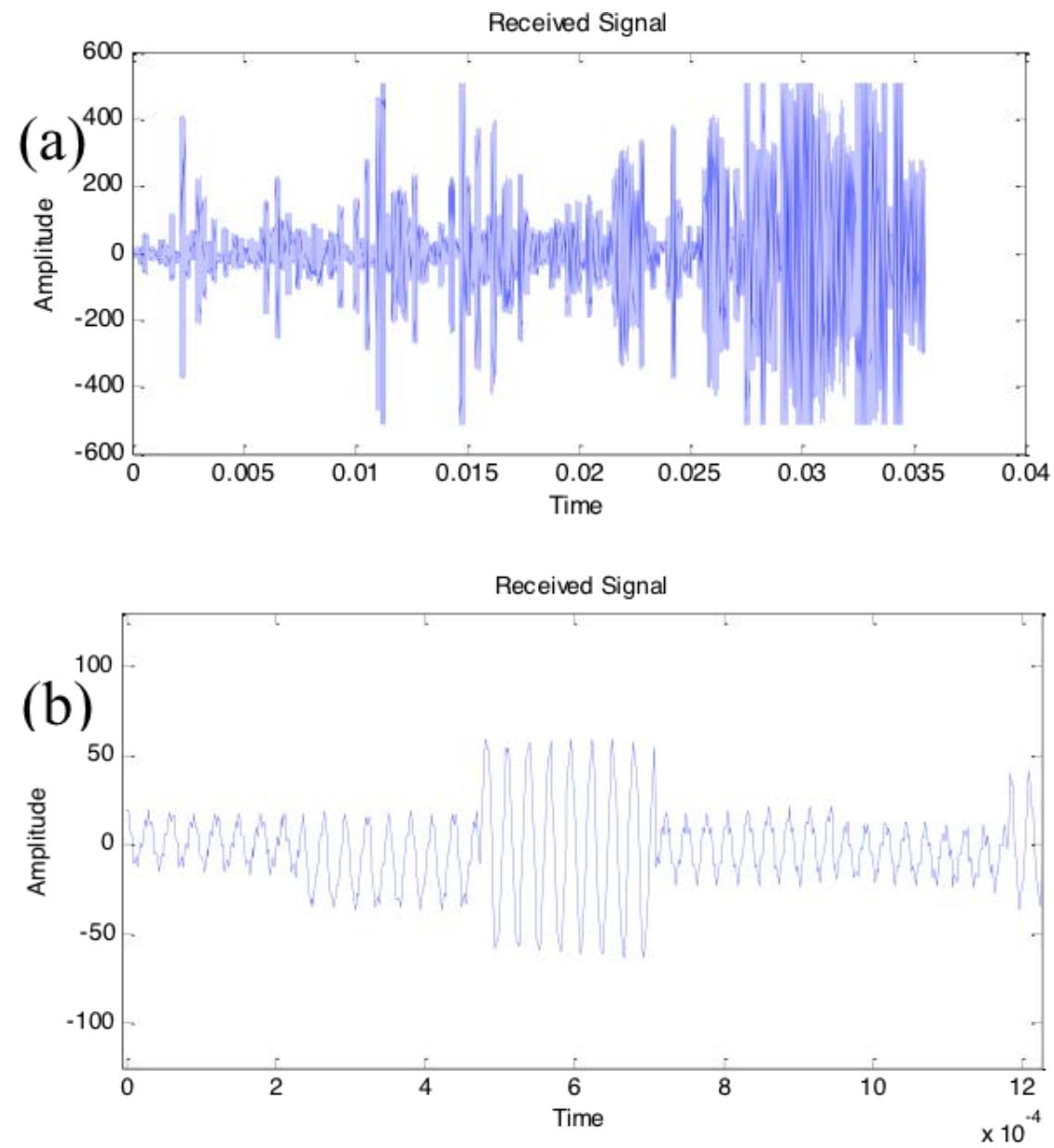

Figure 3-4 (a) Received signal in the time domain (b) Zoom of the received signal in the time domain [138]

The spectrogram of the excitation wave is shown in Figure 3-5. At any time, only one frequency step is excited and the amplitude of the response is measured in the sensor, which could be a second piezo or a non-contact laser scan point. 


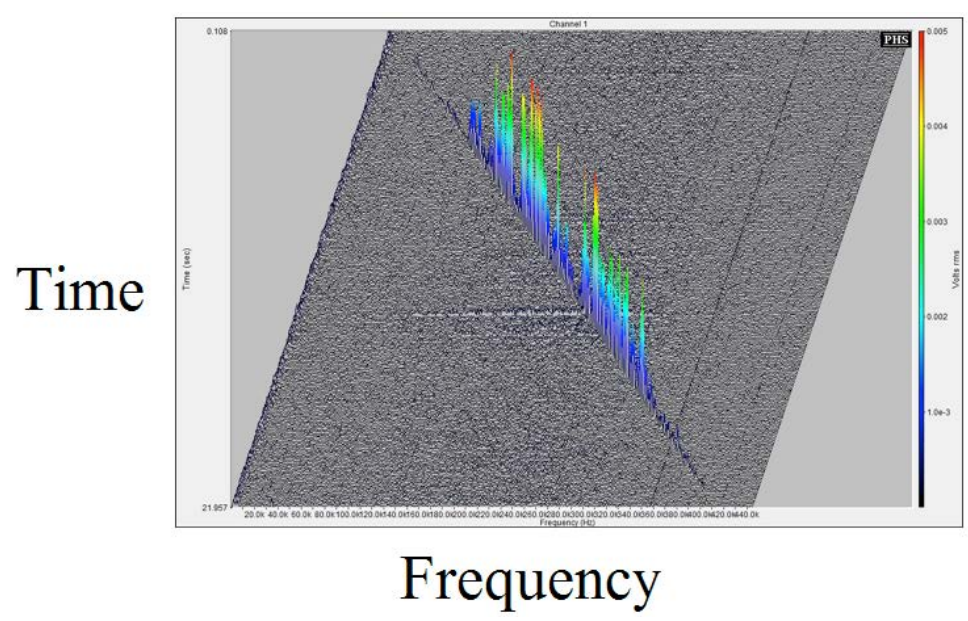

Figure 3-5 Spectrogram of excitation signal

The surface is excited by using a piezoelectric element when the SuRE method is used. Rather than measuring the impedance changes of the ceramic disc, this method excites it with a sine wave voltage. This creates waves on the surface. The wave generation, propagation and amplitude of the received signal at the sensor are different at each frequency. The amplitude variation pattern (spectrum) at the monitored frequency range is the same as long as the surface stays at the same condition. This spectrum changes when the part experiences any changes including defects, cracks, loading, cuts, holes, welding and coating. These spectrum changes can be monitored to detect the listed conditions at the part. In order to compare the spectrums to each other, the sum of square of differences (SSD) method is used. Figure 3-6 shows the increased SSD once the spectrum is altered due to the change in the original condition of the structure. 


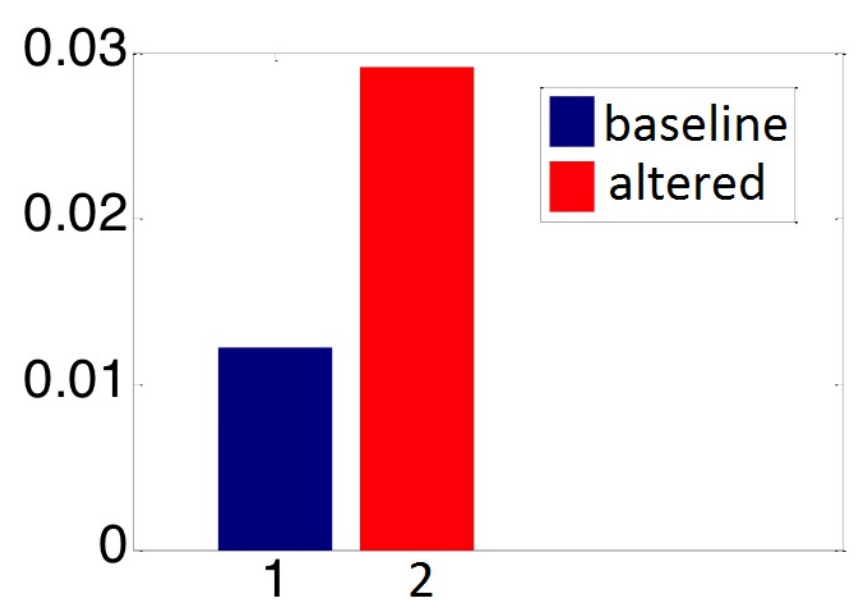

Figure 3-6 The change of squares of the differences (SSD's) in drilling operation

3.4. Computational methods for calculation of the spectrum of the signal

The SuRE method gives flexibility to the user in terms of the excitation signal, actuator, sensor, data acquisition system and signal processing.

\subsubsection{Fast Fourier Transform}

The Discrete Fourier Transform (DFT) [139] is used for computer-based frequency domain analysis. Applications include spectral analysis, de-noising, compression and filtering. The signal requires being discrete in time and having a finite duration before its DFT could be calculated. The discrete Fourier transform of $x[n]$ signal with $\mathrm{N}$ number for samples is calculated from Equation 3-1:

Equation 3-1

$$
\sum_{x=0}^{N-1} x[n] e^{-j \frac{2 \pi}{N} k n}
$$

Fast Fourier Transform (FFT) is a fast computational algorithm for DFT. 


\subsubsection{The Teager-Kaiser Algorithm}

The Teager-Kaiser Algorithm (TKA) [140] is an extremely convenient method for the analysis of pure harmonic signals. In theory, since the TKA estimates the frequency and the amplitude at the same time, the user does not need to keep track of the frequency of the signal separately. However, in practice, the noise, limited information, and difficulty of estimating of two variables from two inputs limited the performance of the method. In addition, calculating the frequency was not necessary with the DSP since the frequency was a known value, sent from the DSP to the Digital Data Synthesizer (DDS).

\subsubsection{The Goertzel Algorithm}

The Goertzel algorithm [21,24] may be used to estimate the amplitude of a pure sine wave signal once the coefficient, which corresponds to the frequency of the signal, is calculated. The estimate can then be updated for each sampling or performed at once after the signals at the same frequency are collected. The estimations are good and theoretically the contamination from the other frequencies is ignored. This is a wellknown and widely used method for DSP applications.

\subsubsection{Sine Wave RMS and Mean Value Methods}

As stated earlier, the monitored output signal is a sine wave. Because of this, the properties of the shape of a sine wave could be exploited for quick and effective calculations. The magnitude of a sine wave is proportional to its RMS value and the average value of either the positive or negative sides [25].

The RMS values is calculated with Equation 3-2: 
Equation 3-2

$$
R M S=\sqrt{\frac{\sum x(i)^{2}}{N}}
$$

Where the " $N$ " is the number of samples of the $x(i)$ signal. The amplitude " $a$ " is calculated by Equation 3-3:

Equation 3-3

$$
a=R M S \sqrt{2}
$$

The relation between the amplitude and the mean of the positive values is calculated with Equation 3-4:

Equation 3-4 $\quad a=\pi\left(\frac{\sum(x(i))}{N}\right)$ where, $x(i)=\begin{array}{cl}0 & \text { if } x(i)<0 \\ x(i) & \text { if } x(i)>0\end{array}$

This is the easiest and fastest way for calculating the amplitude of the output signal. It also turns out to be the most convenient way to achieve this, since DSP analogto-digital converters normally operate by treating voltages less than $0 \mathrm{~V}$ as $0 \mathrm{~V}$.

3.5. Signal Processing for comparing the response magnitudes

\subsubsection{Sum of square of differences (SSDs)}

Once the amplitudes are calculated, the similarity between the two signals may be represented by the sum of the squares of the difference (SSD) between both signals, shown in Equation 3-5: 
Equation 3-5 $\quad S S D=\sum_{i=1}^{n}\left(a_{j, i}-a_{r, i}\right)^{2}$

Where $a_{j, i}$ are the amplitudes of the considered case and they are compared to the baseline $a_{r, i}$.

\subsubsection{Sum of square of differences for multiple sensing point}

The FFT or an amplitude estimation method is then used to calculate the frequency transfer function. Studies have shown that the calculated spectrum for any measurement point on the structure is a signature characteristic and behaves consistently as long as no change occurs on the condition of structure. As soon as the condition changes due to applied load or structural damages, the frequency spectrum changes. To quantify the changes using the SuRE method, the Squared Difference (SD) of frequency spectrums with respect to a reference one is used from Equation 3-6:

Equation 3-6 $\quad D_{m \times n \times p}=\left\|A_{m \times n \times p}-R_{m \times n \times p}\right\|^{2}$

Here, $\mathrm{R}$ and $\mathrm{A}$ are the reference and altered data matrices, including the frequency spectrums of intact and damaged structures. The dimension of each data matrix is $\mathrm{m}$ rows by $n$ columns by p layers. $m$ includes the frequency samples of point $n$ during the $p$ measurement. In this case, the Sum of the Squared Differences (SSD) for each scan point is calculated from Equation 3-7:

Equation 3-7

$$
S_{1 \times n}=\sum_{m} D_{m \times n}
$$

$\mathrm{S}$ is a matrix with the size of measurement points that contains a SSD value for each scanning point. This value quantitatively represents the amount of change in the 
spectrum for each scanning point. Depending on the configuration, scan S could be a onedimensional or two-dimensional array.

\subsubsection{Normalized sum of square of differences (NSSDs)}

In this study, the structure was excited using a piezoelectric element and the surface vibrations were monitored by the scanning laser vibrometer. The reference scan was performed when all the bolts were tight.

The altered scans were performed after one or more bolts were loosened. The change of the compressive forces on the structure surfaces changed the surface response to excitation. To quantify the change, the Squared Difference (SD) of two matrices is calculated with Equation 3-8:

Equation 3-8

$$
D^{2}{ }_{i, j}=\left(A_{i, j}-R_{i, j}\right)^{2}
$$

$\mathrm{R}$ and $\mathrm{A}$ are the reference and altered data matrices obtained by the spectrum analyzer. Data matrices have m rows by n columns, where each column includes the frequency spectrums of a scan point. The frequency range was distributed over the matrix row. The average of the squared differences was calculated with Equation 3-9:

Equation 3-9

$$
\text { Average }=\frac{1}{m n} \sum_{i=1}^{m} \sum_{j=1}^{n} D_{i, j}^{2}
$$

where $\mathrm{m}$ and $\mathrm{n}$ are the number of frequencies and the number of scan points, respectively. The Normalized Squared Differences (NSD) was obtained from dividing the squared differences by the average in Equation 3-10: 
Equation 3-10

$$
\bar{D}_{i, j}=\frac{D_{i, j}^{2}}{\text { Average }}
$$

The normalized differences for each scan point could be averaged versus the frequency range to obtain the Normalized Sum of Squared Differences (NSSD), and in fact this criterion was an array with the size of scan points:

Equation 3-11

$$
\bar{S}_{i}=\frac{1}{n} \sum_{j=1}^{n} \bar{D}_{i, j}
$$

$\bar{S}$ is a matrix with the size of the scanning grid that contains a normalized value for each scanning point. This normalized value quantitatively represents the amount of change in the spectrum for each scanning point. Depending on the dimension of the scan grid, $\bar{S}$ could be a one-dimensional or two-dimensional array and could be represented in different graphical ways.

\subsection{Experimentation setup}

In this study, three different approaches were used for implementing the SuRE method. In order to excite surface waves on the beam, an APC piezoelectric model D.750"-2MHz-850 WFB was attached to the middle of the plate. All the approaches excited the surface with a piezoelectric element (Figure 3-8). To remove oil and any other possible contaminants, the surface of aluminum plate was cleaned with acetylene, ethanol and water. The bonding agent was LOCTITE Hysol Product E-30CL epoxy adhesive, with a curing time of 24 hours. An applicator gun simultaneously mixed and dispensed the bonding agents from a dual-cylinder cartridge by passing the ingredients 
through a mixing nozzle. Normally, a frame is used to position the specimen in front of the laser head (Figure 3-7). The scan points are marked on the clamp to make sure that at every step of experimentation the same points would be scanned. A RIGOL DG1022 function/arbitrary waveform generator with a maximum peak-to-peak amplitude of 20 volts generates the waves. In order to have a higher signal-to-noise ratio in measurement points, the waveform is amplified by 5 times through passing a TEGAM power amplifier model 2348. The laser scanning Doppler vibrometer (LSDV) model Polytech PSV-400 remotely measures the surface vibrations from a grid of scan points on the aluminum plate. Due to the limitation of sampling frequency of the A/D converter of laser junction box, an external data acquisition system was used. Data Translation simultaneous A/D convertor model DT9832-A was employed to capture the peak holds of the transfer function for the frequency input sweep sine wave. The maximum sampling rate of the device was set to 1 million samples per second. This allowed a maximum frequency of $400 \mathrm{kHz}$ to be sampled. Since the SuRE algorithm requires the frequency domain data, the FFT of the input data was used. The DT9832-A has a built-in FFT package. 

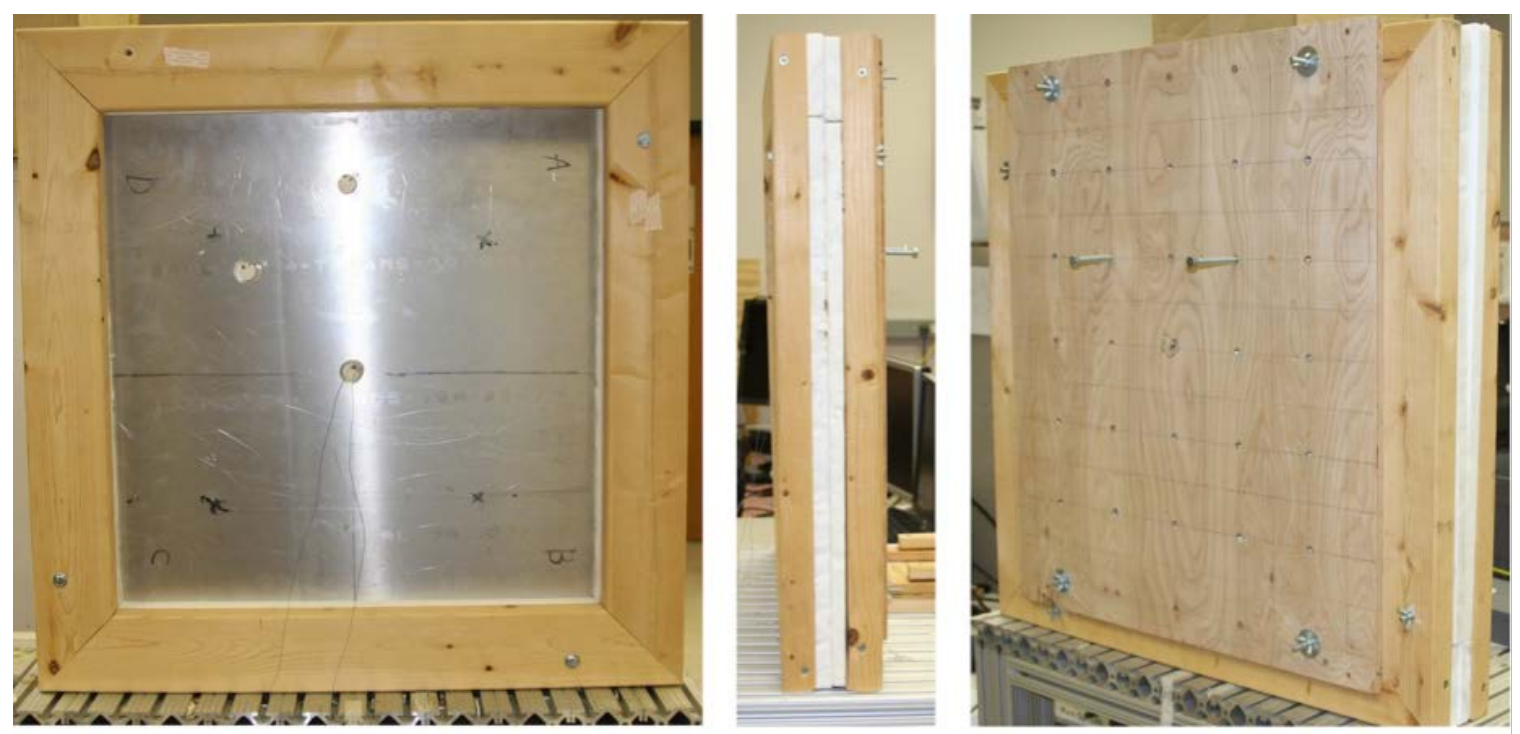

Figure 3-7 An example of the frame used to hold the plate for laser scan

The first two approaches monitored the surface vibrations with a second piezoelectric element but used different approaches for the analysis of the monitored signal. The third approach used the scanning laser vibrometer to monitor the surface waves at a grid on the surface. They will be briefly discussed in this section.

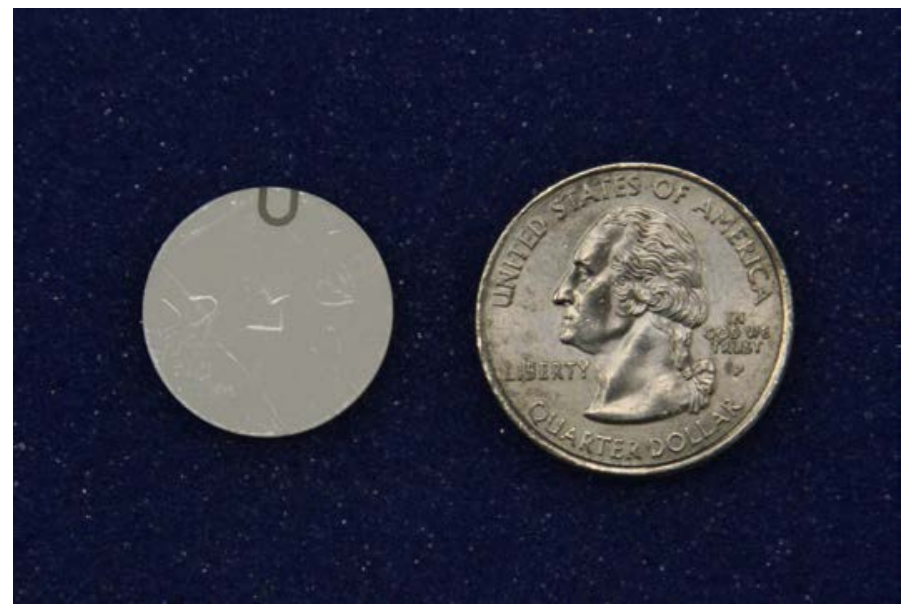

Figure 3-8 3/4” diameter APC PZT model D-.750"-2MHz-850 WFB 


\subsubsection{Implementing the SuRE method with Digital Signal Processors (DSP):}

Figure 3-9 shows the schematic of implementing SuRE method. It uses the Signal Analyzer or Microchip dsPIC33FJ64MC802 DSP mounted on a Microstick development board. One limitation of the DSP was that it could not generate good quality sine waves through Pulse Width Modulation (PWM) at the frequencies desired. A Midnight Design Solutions Direct Digital Synthesizer (DDS) board, the LLC DDS-60 Daughterboard, was used to generate the sine waves. The generated sine waves were passed through AD826AN operational amplifier to boost signal power and applied to the piezoelectric element attached to the structure. The signal of the other piezoelectric element on the structure was sampled through the DSP after it was conditioned with another operational amplifier (AD826AN). Sampling was performed at the 1.1 million samples per second. The DSP selected the test frequencies, collected data, calculated the amplitudes, compared them with the reference signal and estimated if the structure is under a compressive force or not.

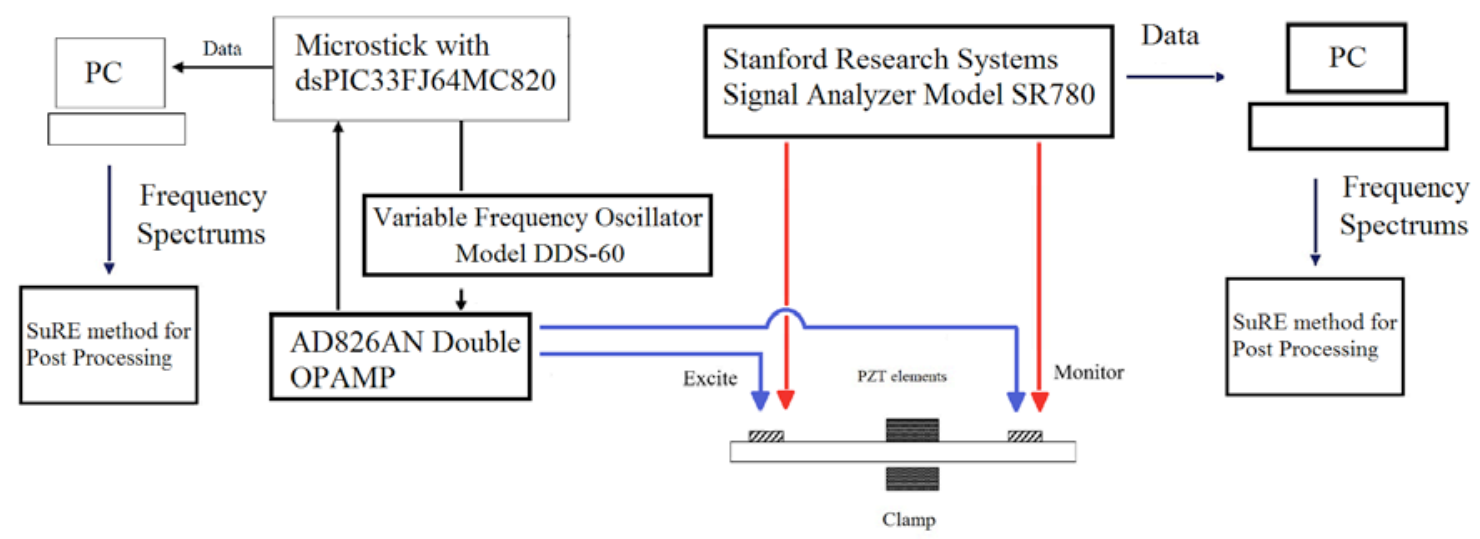

Figure 3-9 The experimental setup for the SuRE method using a signal analyzer and a DSP circuit

The embedded device that has been developed based on PIC's 16-bit Digital Signal Controller is shown in Figure 3-10. A Digital Signal Controller (DSC) is a single- 
chip, embedded controller that integrates the control attributes of a Microcontroller (MCU) and the computation capabilities of a Digital Signal Processor (DSP). They are designed to execute digital filter algorithms and high-speed precision digital control loops. The model dsPIC33FJ128MC802 has a maximum analogue to digital conversion speed of up to1.1Msps which covers the frequency range of interest for our experiments.

The Universal Asynchronous Receiver Transmitter (UART) module was used to establish a communication line with the microcontroller. This way the raw data was transferred to the PC to post processing using the SuRE method. Each data file included two columns to include the excitation frequency and corresponding monitored amplitudes.

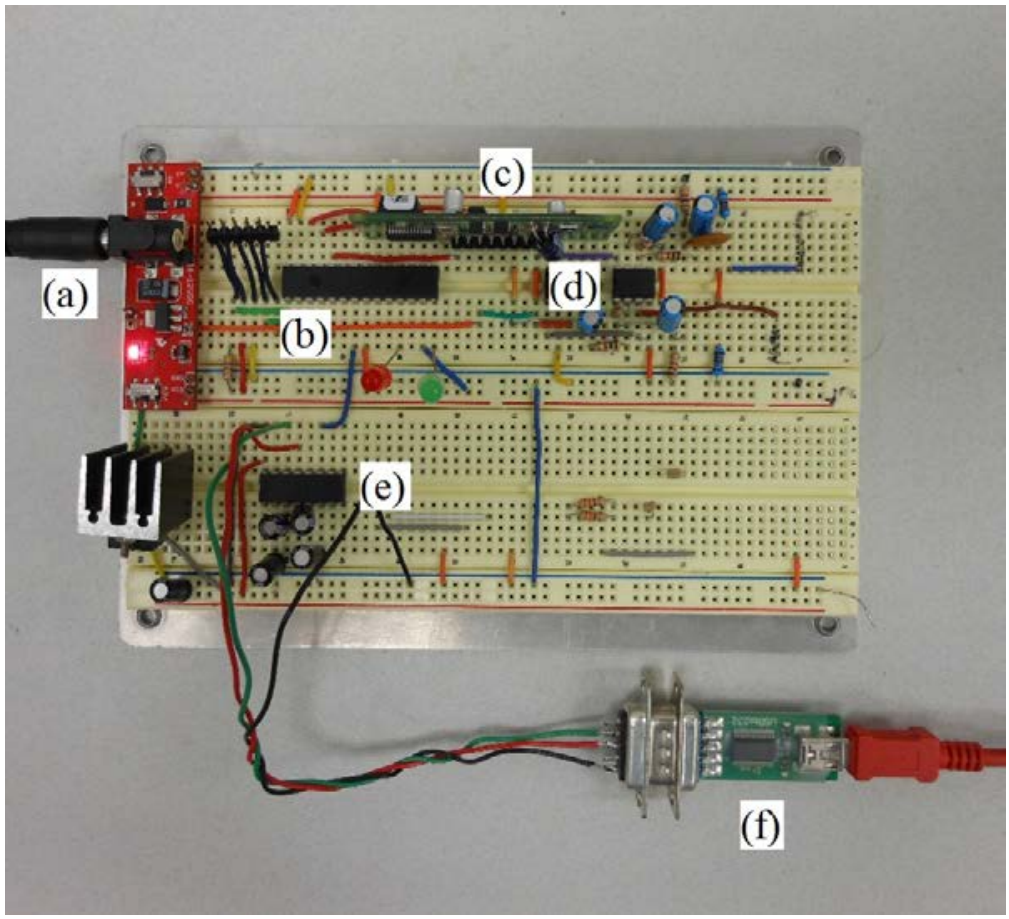

Figure 3-10 Embedded device for the SuRE method (a) power source, (b) dsPIC33f, (c) DDS-60 (e) OPAMP (d) UART (e) R232-USB adapter 
3.6.2. Implementing the SuRE method with spectrum analyzer:

Surface waves were generated using a piezoelectric element. Second Piezoelectric element was used to monitor the surface waves at the other end of the beam. The excitation signal was generated and the monitored signal was analyzed by using the Stanford Research Systems (SRS)-SR780 spectrum analyzer shown in Figure 3-11. The spectrum analyzer generated the sweep sine wave and calculated the magnitudes of the transfer functions between the excitation signal and the response at the $1-100 \mathrm{KHz}$ range.

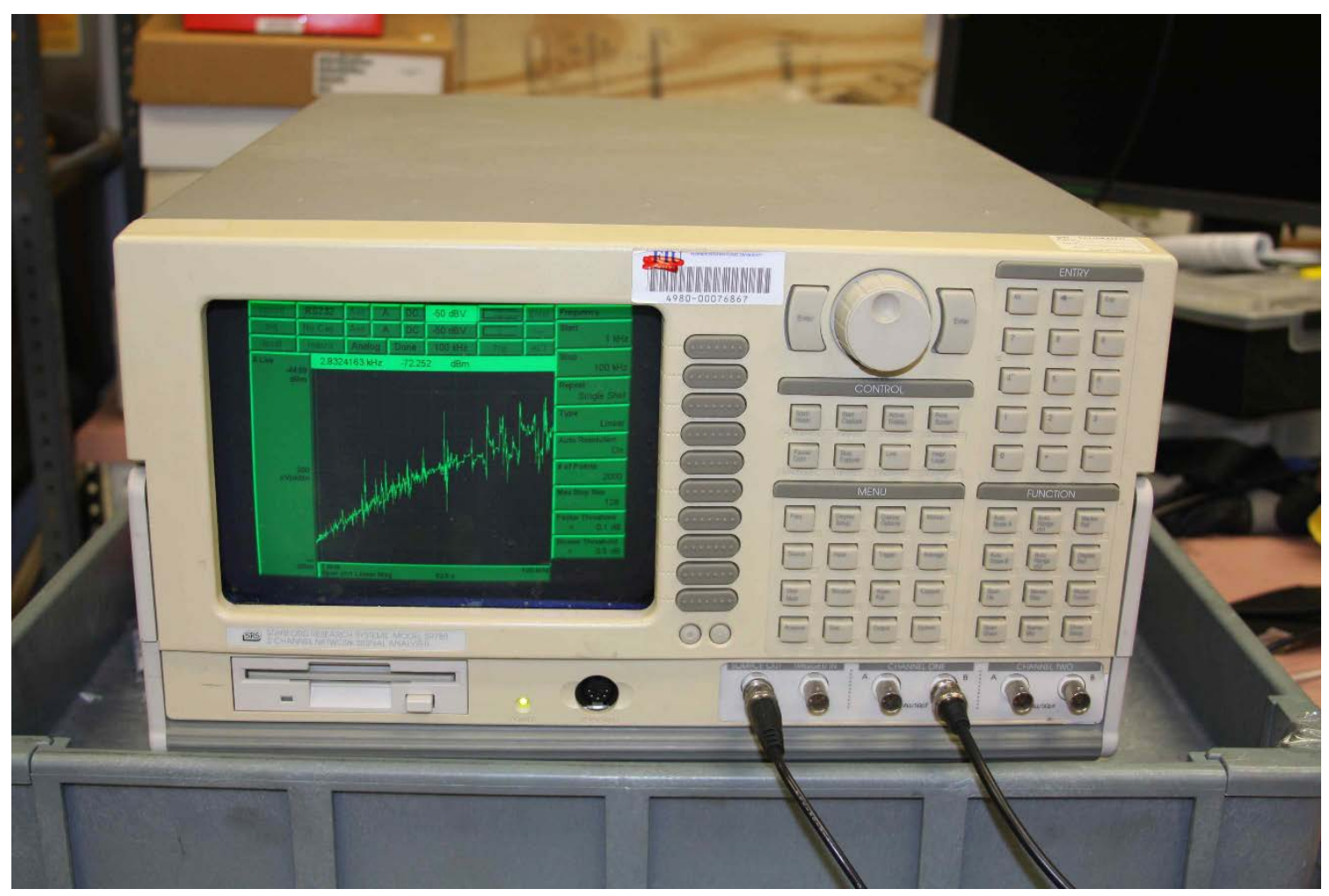

Figure 3-11 Stanford Research Systems (SRS)-SR780 spectrum analyzer. 


\subsubsection{Monitoring with a scanning laser vibrometer (Laser Doppler Vibrometer):}

The Polytech PSV-400 Scanning Laser Vibrometer (Laser Doppler Vibrometer), shown in Figure 3-12, was used for measurement of the surface waves. The sensitivity of the piezoelectric element is much higher than the scanning laser vibrometer. However, the scanning laser vibrometer may measure the oscillations at much more small areas compare to the piezoelectric elements and the vibrations of hundreds of points may be measured without attaching anything to the surface. Typically a laser system will have to perform multiple scans at a single point, taking many averages, to be as accurate as a piezoelectric-based system.

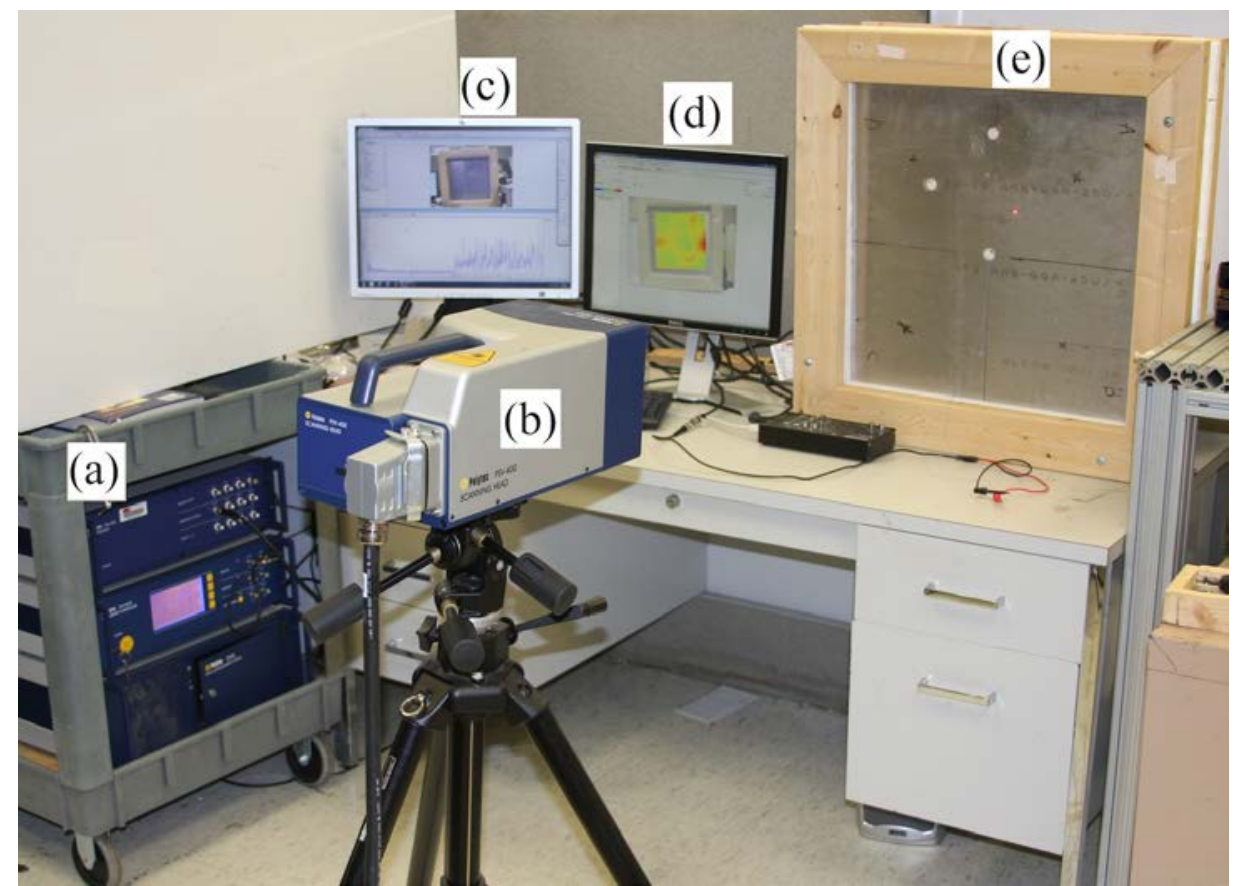

Figure 3-12 PolyTech PSV-400 Laser Doppler Vibrometer experimental set up; (a) Vibrometer Controller (b) Scanning Head (c) PSV software (d) the SuRE method colormap (e) Aluminum plate 


\section{SIMULATION OF THE SURFACE RESPONSE TO EXCITATION METHOD}

FOR STRUCTURAL HEALTH MONITORING

\subsection{Introduction}

In this study, COMSOL was used to simulate the surface response to excitation method (SuRE method). The SuRE is a frequency-domain technique similar to electromechanical impedance method where the changes in frequency transfer function are considered as damage indicators. An aluminum beam with a piezoelectric element bonded is modeled using COMSOL's piezoelectric module. The frequency spectrum of the structure is monitored through a frequency range of $(20 \mathrm{kHz}-400 \mathrm{kHz})$. A frequency domain sweep study was performed to simulate the sweep sign generation. A set of probe points measured the response to simulate the experimentations. In experimental tests laser scanning vibrometer measures the surface oscillations from a grid of scan points. The changes in spectrums of probe points due to milling, drilling and cutting operations are investigated using sum of squared differences (SSD) method.

\subsection{Implementation of SuRE Algorithm}

In this study, COMSOL simulates laser vibrometer monitoring surface accelerations during various metal cutting operations through a grid of scan on an aluminum beam. Figure $4-1$ shows a schematic of aluminum beam, PZT exciter; $5 \times 7$ probe points and the metal cutting operations. 


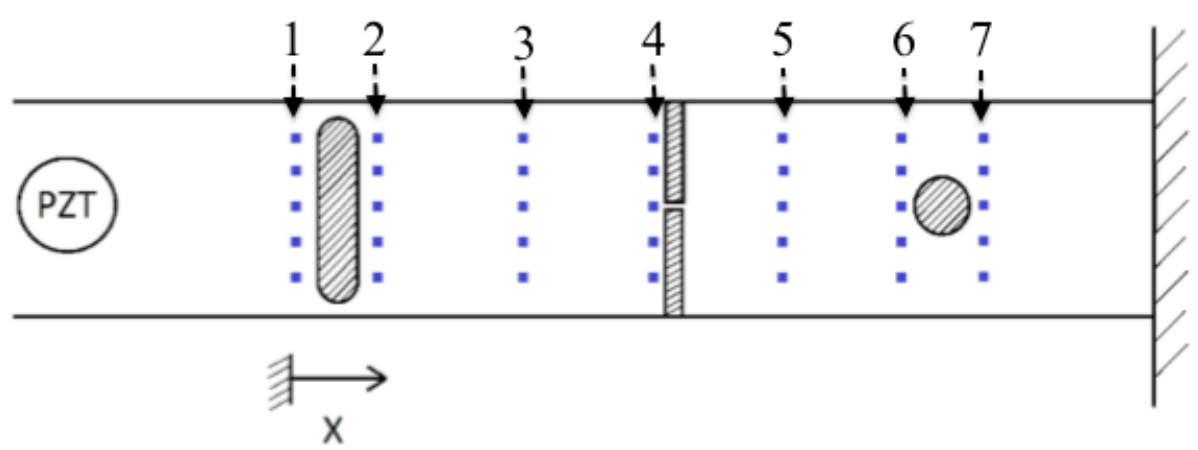

Figure 4-1 Schematic of aluminum beam with PZT exciter, scan probes and drilling, cutting and milling operations.

Based on the SuRE method first a baseline data was measured probe points. Subtracting material from the model simulated each manufacturing operations. Based on the SuRE method described in this section, the SSD values were calculated and compared to those of baseline at every step of operation. The behavior of surface waves and SSD values and their response to each operation were studied.

4.3. Use of COMSOL Multi-physics for Sure-based Manufacturing Process Performance Monitoring Method

Table 4-1 Dimensions of beam, piezo and operations

\begin{tabular}{llll}
\hline \multicolumn{1}{c}{ Property } & Value & \multicolumn{1}{c}{ Property } & Value \\
\hline Length of Plate & 36 [in] & Width of Cut & .05 [in] \\
\hline Depth of Plate & 2 [in] & Depth of Cut & 0.95 [in] \\
\hline Thickness of Plate & $(1 / 16)$ [in] & Location of Cut & $26.2[\mathrm{in}]$ \\
\hline Radius of Piezo & 0.375 [in] & Width of Milling & 0.75 [in] \\
\hline Thickness of Piezo & 0.040 [in] & Depth of Milling & 1.5 [in] \\
\hline Radius of Hole & 0.5 [in] & Location of Mill & 22 [in] \\
\hline Location of Hole & 30 [in] & & \\
& & & \\
\hline
\end{tabular}


The model used in this study was an aluminum specimen with (2in) $\times(36 \mathrm{in})$ beam and (1/16in) thickness. The piezoelectric is attached to the middle of the beam. Table 4-1 shows the dimension of materials and operations being used in the model:

The model was created in COMSOL's piezoelectric device module and investigated in a frequency domain study. The beam material is aluminum with density of $2700 \mathrm{~kg} / \mathrm{m}^{\wedge} 3$, modulus of elasticity of $70 \mathrm{GPa}$ and Poisson’s ratio of 0.33 . The piezoelectric element is Lead Zirconate Titanate (PZT-4) type with density of 7500 $\mathrm{kg} / \mathrm{m}^{\wedge} 3$.

Although the physics of this study is piezoelectric devices, the physics of beam is set to linear elastic material to reflect the multi-physics nature of this study. According to experimentation boundary condition of both ends of the beam is fixed constraints and everywhere else is free. The lower surface of piezoelectric element is considered as ground with a zero charge and upper surface is charged with the piezoelectric element is charged with a 20 Volts. Figure 4-2 shows the geometry of the model of this study. 

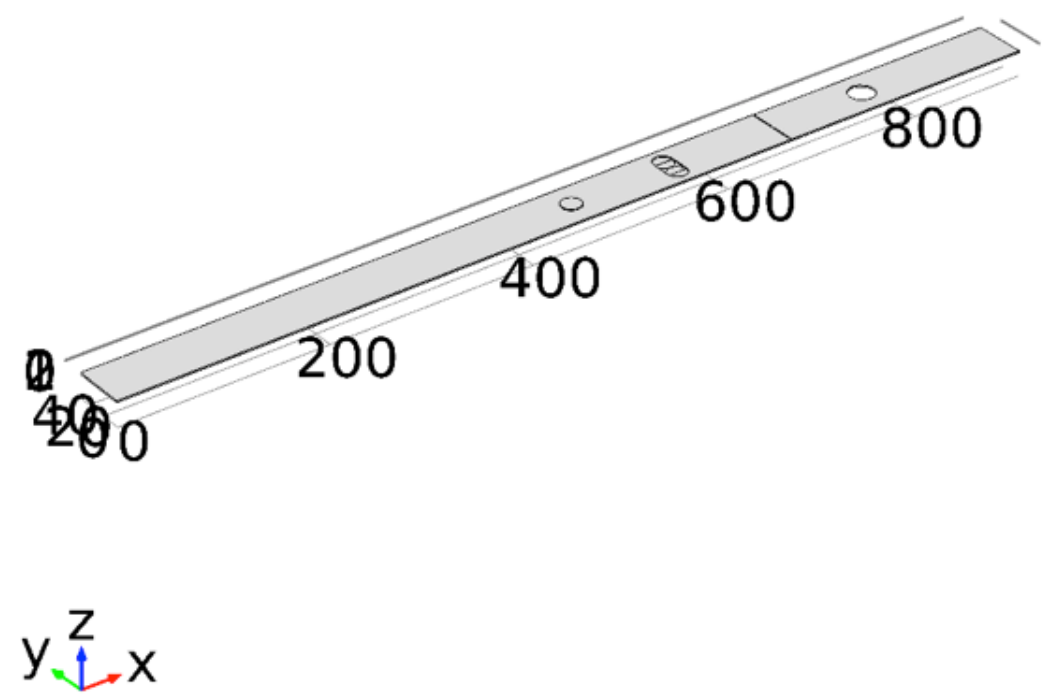

Figure 4-2 Aluminum beam model with a PZT element in center; drilling, cutting and milling are simulated.

The model meshed with free tetrahedral elements. The minimum mesh size was set $50 \mathrm{~mm}$ and the maximum mesh size was set 500mm (Figure 4-3).

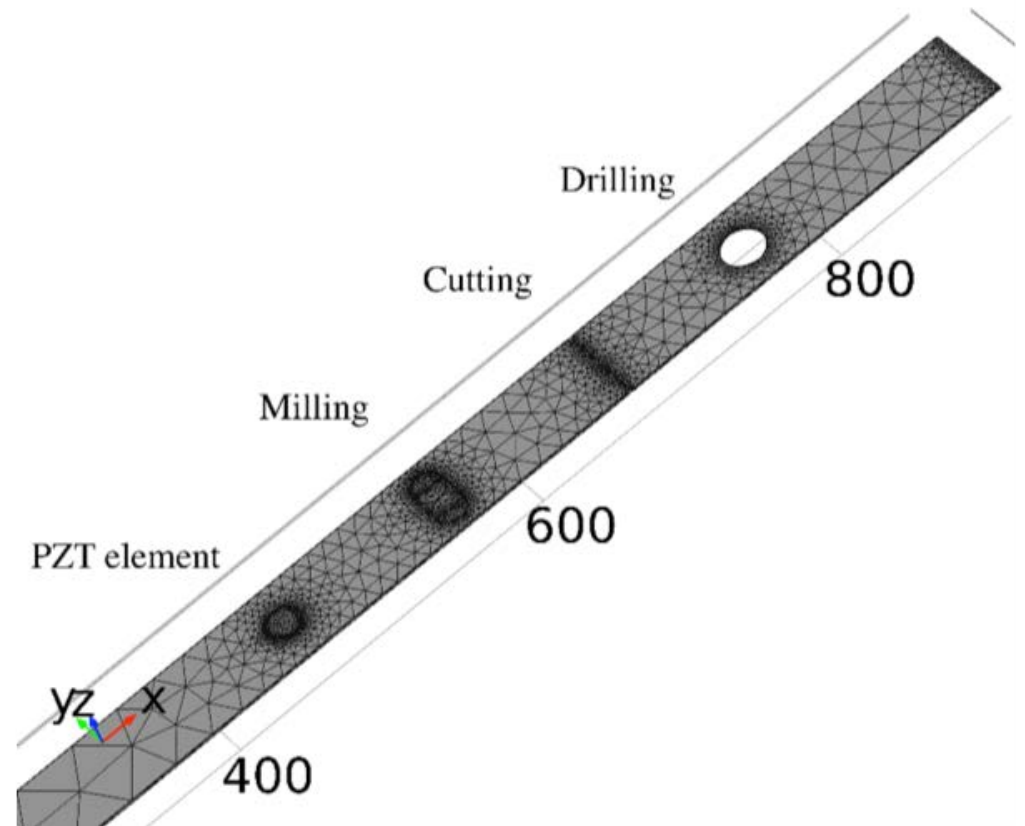

Figure 4-3 Meshed model with free tetrahedral elements 
The surface response to excitation method excites a sweep of high-frequency surface-guided waves on the work-piece and measures the instantaneous Fast Fourier Transform of the response in the probe points. To simulate this process of the SuRE method, a frequency domain study was chosen and the frequency range of $20-400[\mathrm{kHz}]$ with step of $1[\mathrm{kHz}]$ was performed. The initial values of displacement and velocity for all points were zero. The amplitudes of response and their time derivatives of each probe point were obtained from the solution. The final solution includes the spectrum of transfer function for 35 probe points. The baseline data was obtained when the beam was intact and following data sets obtained after each of the drill, cut and mill was performed.

\subsection{Simulation Results}

Figure 4-4 shows a typical spectrum from a probe point resulting from simulation. The concept of the SuRE method is based on assuming consistency of these spectrums in the absence of damages. Another characteristic of method is the local sensitivity of spectrum to the structural damages.

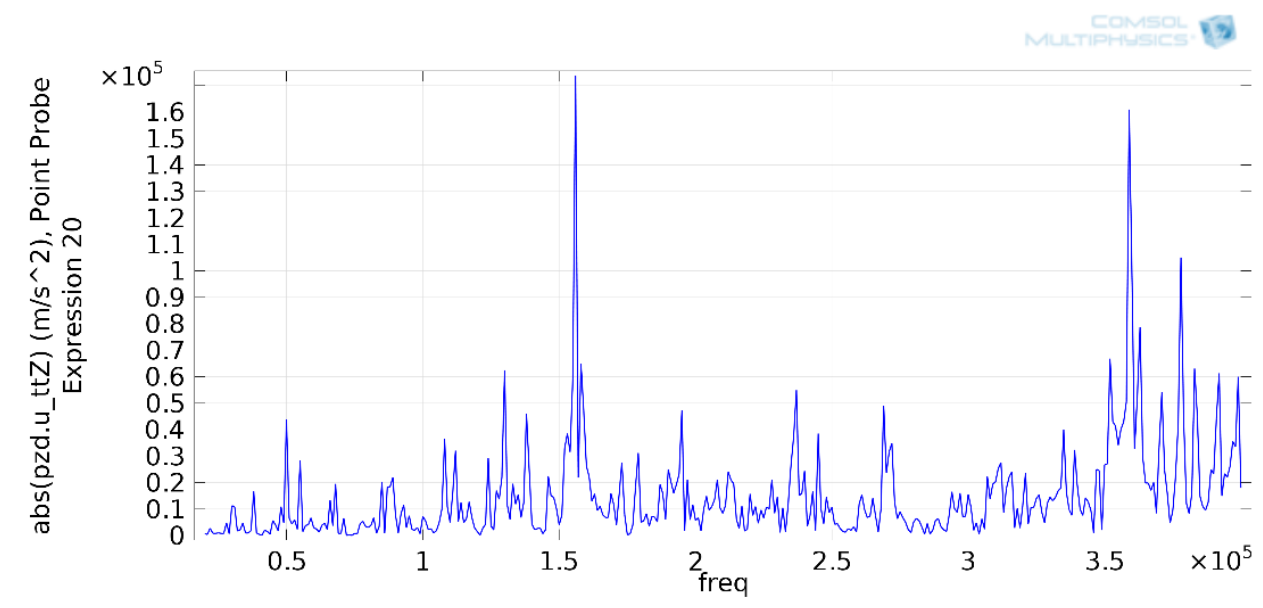

Figure 4-4 Accelerations vs. frequency in spectrum from a probe point 
In presence of a fatigue cracks the spectrum of probe points adjacent to crack will influenced more than the farther ones. This phenomenon is demonstrated in Figure 4-5 (a) and (b). Both figures compare the spectrums of before and after the drill hole was introduced to the beam. In Figure 4-5(a) the probe point is located on the $2^{\text {nd }}$ probe column and in Figure 4-5(b) the probe point is located on the $6^{\text {th }}$ probe column.
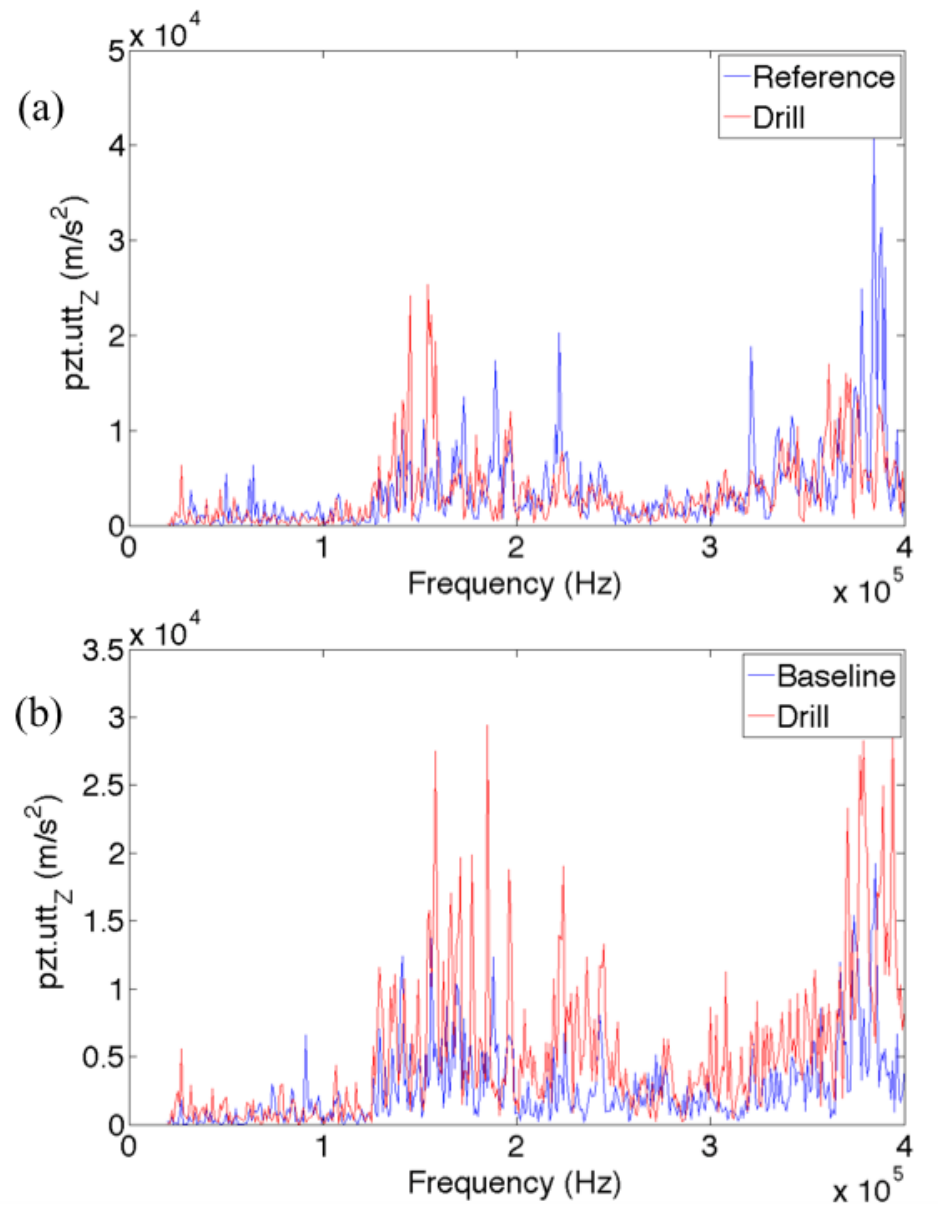

Figure 4-5 (a) Spectrums of before and after the drill hole from a probe in $2^{\text {nd }}$ column (b) Spectrums of before and after the drill hole from a probe in $6^{\text {th }}$ column

Both spectrums are influenced due to the presence of drill operation but the probe point in the 6th column is showing much more deviation from its initial condition. 
According to schematic shown in Figure 4-1 the drilling operation was performed between $6^{\text {th }}$ and $7^{\text {th }}$ scan probes. Therefore, this phenomenon reflects the locality property of transfer function spectrums.

\subsubsection{Drilling}

Since the study was a frequency domain study, the solution set includes a set of displacements for each frequency starting from $20[\mathrm{kHz}]$ to $400[\mathrm{kHz}]$ with $1[\mathrm{kHz}]$ step. Figure 4-6 shows the solution for displacements of the beam for the 1st frequency step, $20[\mathrm{kHz}]$.

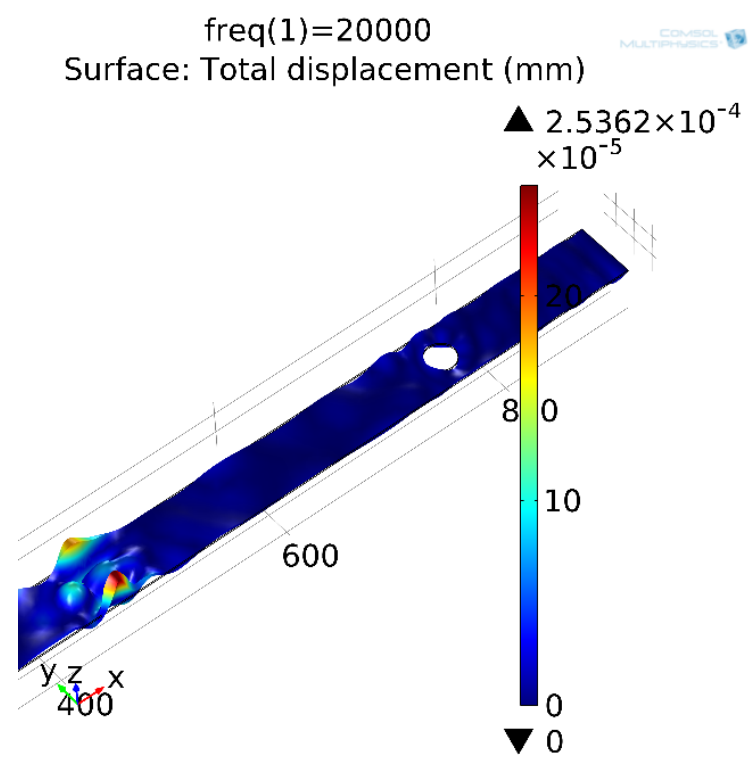

Figure 4-6 The drilling surface displacements solution for the 1st frequency step; $20[\mathrm{kHz}]$.

While there is significant vibrations in the vicinity of piezoelectric, no considerable local change in the oscillations close to hole has occurred for the $20[\mathrm{kHz}]$. The advantage of the SuRE method is that it is not limited to a single frequency and a 
range of frequencies is examined. Figure 4-7 shows the exaggerated displacements on the beam for 22[kHz].

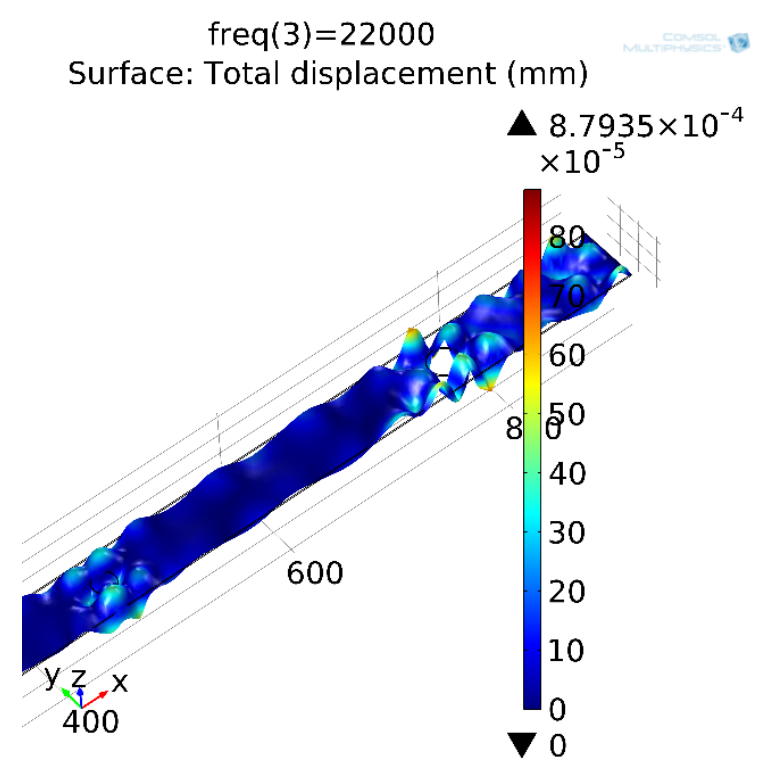

Figure 4-7 The drilling surface displacements solution for the 3rd frequency step; $22[\mathrm{kHz}]$.

In $22[\mathrm{kHz}]$ significant vibrations are surrounding the hole which means that this frequency to the creation of hole in this specific location of the beam. The SuRE method compares the spectrums of all probe points to the baseline ones using the sum of squared differences method as described in chapter 1 . The bar diagram in Figure 4-8 shows the SSD values for all scan points after drilling. 


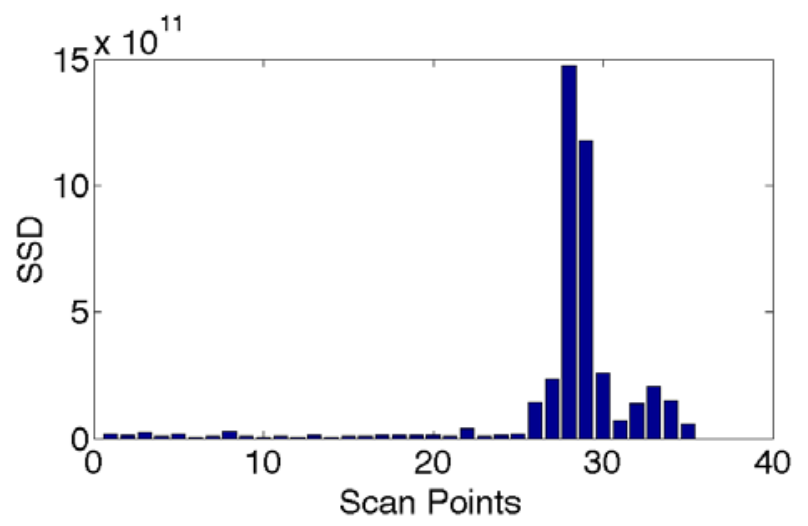

Figure 4-8 Bar diagram of SSD values vs. scan points for drilling.

The maximum SSDs are occurred in probe points 25-35. These probes are located in the 6th and 7th scan columns that are surrounding the drill hole. Figure 4-9 gives another perspective of behavior of SSD values by demonstrating a color-map of them over the surface area of beam.

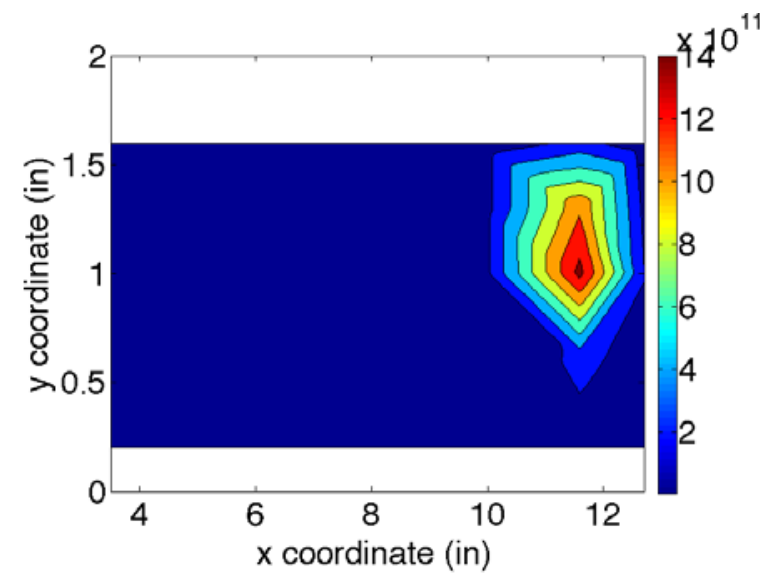

Figure 4-9 Color-map of SSD values vs. scan surface for drilling.

The color-map reveals the drill hole in the correct location. Prior to this only the presence of machining operation could be estimated by monitoring the changes in the SSD values. Similar color-maps could be used for the SuRE-based manufacturing process monitoring systems to monitor the correct location of operation. A simple image processing could locate the position of red spot and compare it to programmed machining 
operation. Depending on the number for data points even it is possible to estimate the dimensional accuracy and surface quality using a higher definition of color-map.

\subsubsection{Cutting}

The cutting operation was a symmetric double cut type from ends of the beam's width. But the cut was not all the way through and small part still was connecting the left and right sides to the cut to each other. In this case, three distinguishable behaviors were observed in the way the surface waves reacted with the cutting edges.

Figure 4-10, $25[\mathrm{kHz}]$ case, shows the beam in which the waves passed through the narrow connection in the cut without a considerable interaction with the cutting edges.

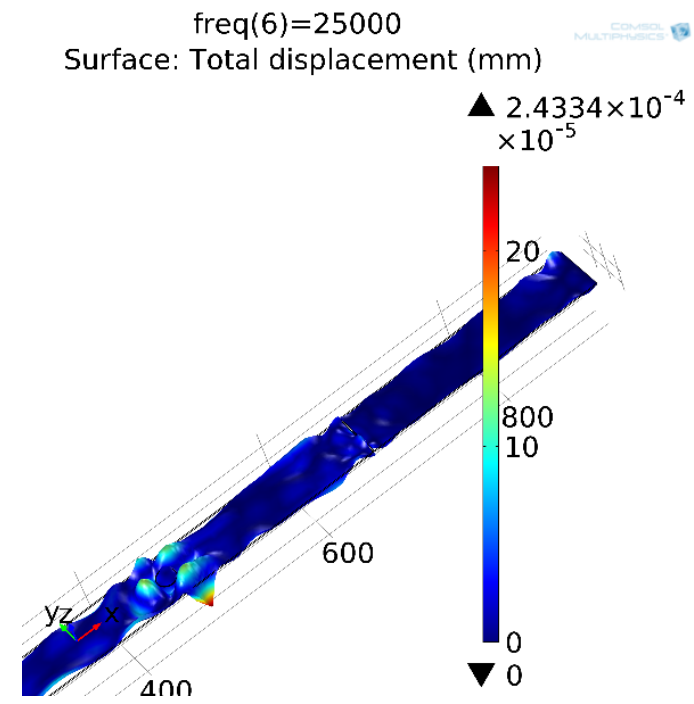

Figure 4-10 The cutting surface displacements solution for the 6th frequency step; 25[kHz]; waves passed thought cut without considerable interaction. 


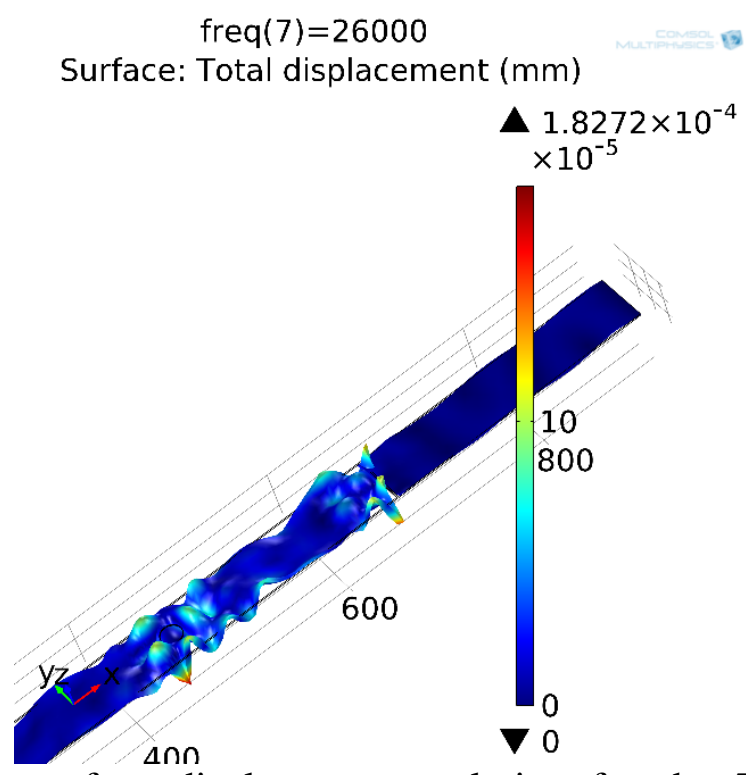

Figure 4-11 The cutting surface displacements solution for the 7th frequency step; $26[\mathrm{kHz}]$; waves reflected from cutting edge.

In Figure 4-11 26[kHz] case, surface waves are reflected from the cutting edges creating a considerable oscillations on the left side of the edge while the right side is pretty much calm. In the $3^{\text {rd }}$ case the waves passed thought the cutting edge and created magnified amplitudes around the cutting edge. This case is shown in Figure 4-12 for $34[\mathrm{kHz}]$. 


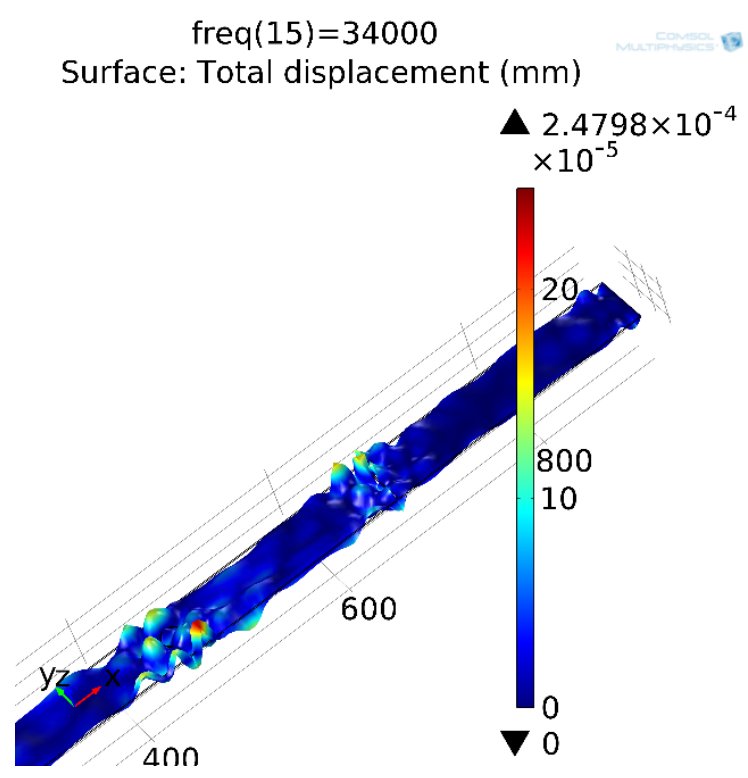

Figure 4-12 The cutting surface displacements solution for the 15th frequency step; $34[\mathrm{kHz}]$; waves passed thought cut with showing interaction.

In Figure 4-13 the color-maps of SSD values highlighted the cutting edges correctly in the middle of scan area.

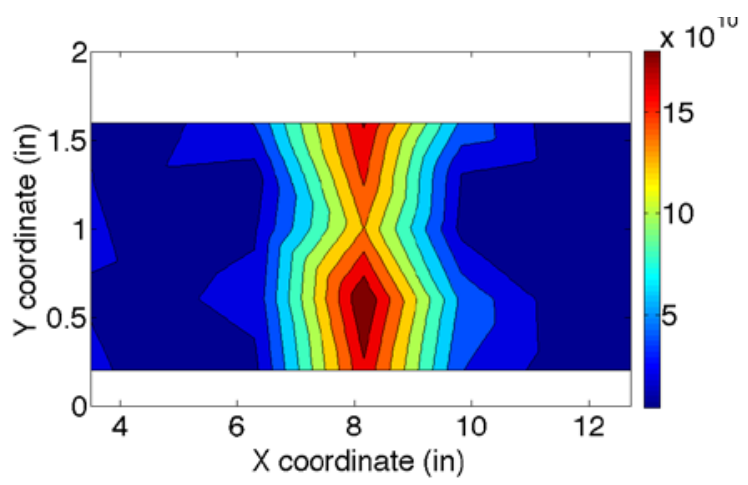

Figure 4-13 Color-map of SSD values vs. scan surface for cutting.

The bar diagram of SSD values in

Figure 4-14 shows the values increase in probe points close to the cutting edges. 


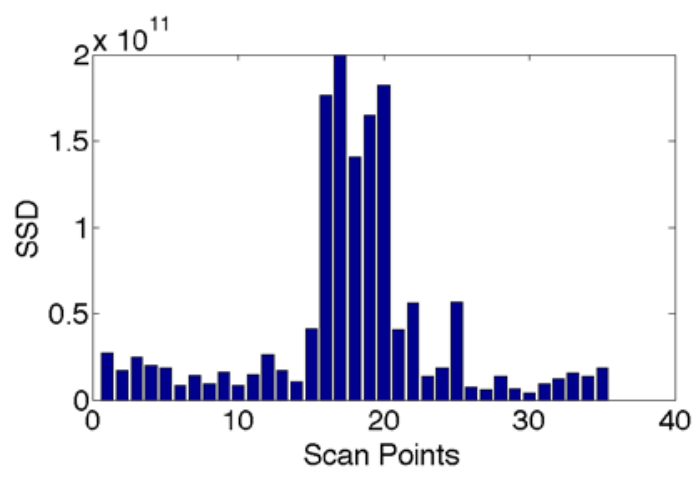

Figure 4-14 Bar diagram of SSD values vs. scan points for cutting

\subsubsection{Milling}

The milling operation was the closest operation to the location of piezo in the middle of beam. Figure 4-15 shows that the oscillations adjacent to milling operation are combined to those around the piezo. This phenomenon shows that in order to have consistent measurements the location of piezo element should be chosen in a reasonable distance from manufacturing operations.

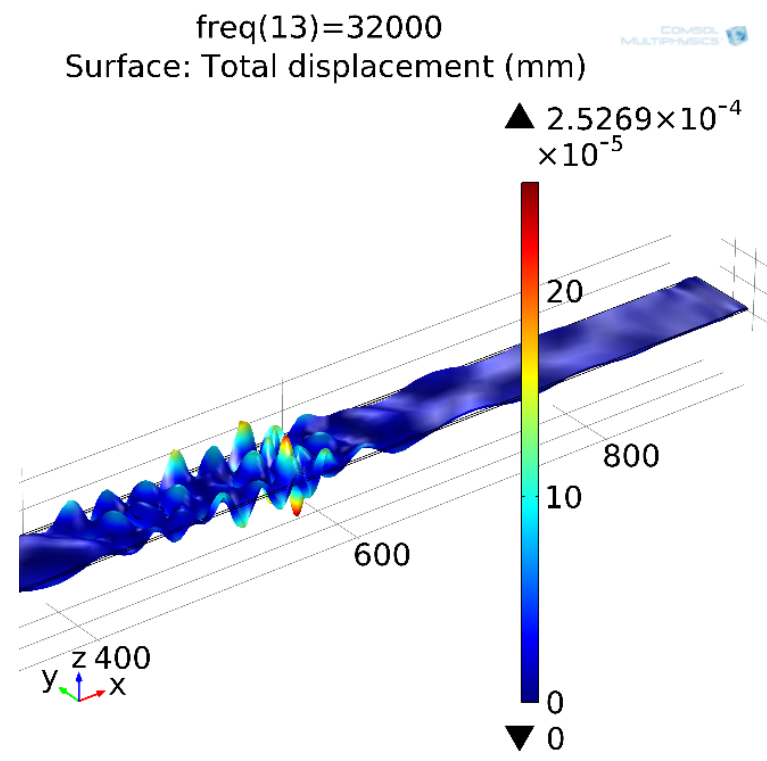

Figure 4-15 The cutting surface displacements solution for the 14th frequency step; 32[kHz]; waves interacted with milling are mixed with vibrations around piezo 
Figure 4-16 shows the SSD values for scan points and their color-map over for the case of milling operation.

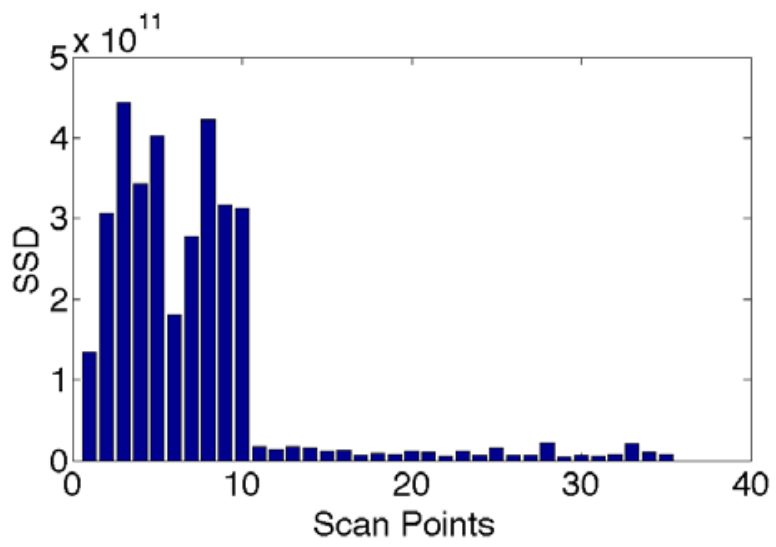

Figure 4-16 Bar diagram of SSD values vs. scan points for milling.

\subsection{Conclusions}

COMSOL provided an insight of behavior of surface guided waves and their integration with cutting edges. It revealed the surface oscillations for every frequency step and the probe points were able to generate spectral data similar to those in the SuRE method. In chapter 6 COMSOL proves to be consistent with experimental data where experimentation is designed with similar condition on the same specimen that was studied in this chapter. The results of this simulation could be used to identify the reliability of the SuRE method for complicated geometries and various damage scenarios in SHM application. Also based once the reaction of surface waves with the damage type is revealed in each frequency, it is possible to identify the optimum frequencies for monitoring purposes. 
5. IMPLEMENTATION OF THE SURE METHOD FOR STRUCTURAL HEALTH MONITORING (SHM) APPLICATIONS

5.1. Monitoring the Load Condition of a Beam Remotely by Using Scanning Laser Vibrometer

\subsubsection{Introduction}

The loading location estimation capability of the surface response to excitation (SuRE) method was studied for a beam. Load was applied to different locations of a beam. Surface was excited with a piezoelectric element. The scanning laser vibrometer was used to monitor the surface response characteristics of the entire surface. These characteristics were presented with 2-D pseudo-colored plots. The study indicated that the surface response characteristics change with application of a significant load to a beam, and the sensitivity of the scanning laser vibrometer is satisfactory to monitor surface waves. These changes may be detected almost at the entire beam. The maximum change of the surface response characteristics is around the load application point. Various sensors may be used to detect load and to estimate its location.

\subsubsection{Experimental setup}

The experimental set up was shown in Figure 3-12. The experimental setup was prepared to study the surface response of an aluminum plate when loads were applied to the different points of it while a piezoelectric element excited the surface.

An APC piezoelectric element with 0.75 inch diameter was attached to an aluminum beam with 2” width, 1/32” thickness and 36” length. LOCTITE Hysol Product E-30CL epoxy adhesive was used to attach the piezoelectric element to the aluminum 
beam. Two additional beams were prepared similarly by using the aluminum beams with 1/16” and 1/8” thicknesses.

The aluminum beams were installed into the experimental setup presented in Figure 5-1. The frame was holding the aluminum beam. There were 10 bolts in the frame to apply load to the beam at the desired point(s). Polytec PSV-400 junction box generated the excitation signal. The sweep sine wave was generated in $20-40 \mathrm{kHz}$ frequency intervals to keep the signal to noise ratio maximum. The signal was magnified by using TEGAM power amplifier model 2348 to the $30 \mathrm{Vpp}$ amplitude. The piezoelectric element was excited with the magnified signal. The PSV-400 Polytec scanning laser vibrometer directed the laser beam to the programmed grid points and measured the surface vibrations.

(a)

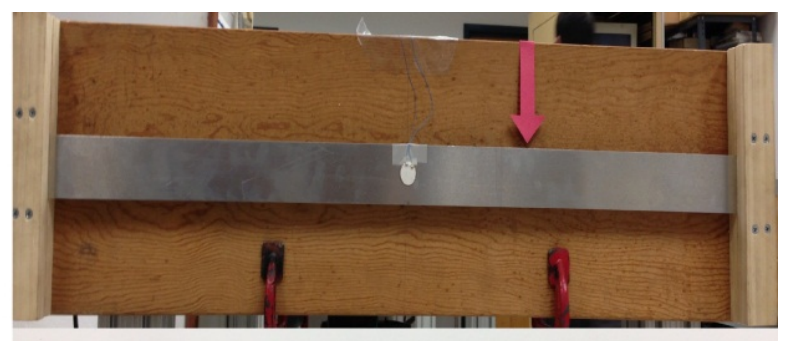

(b)

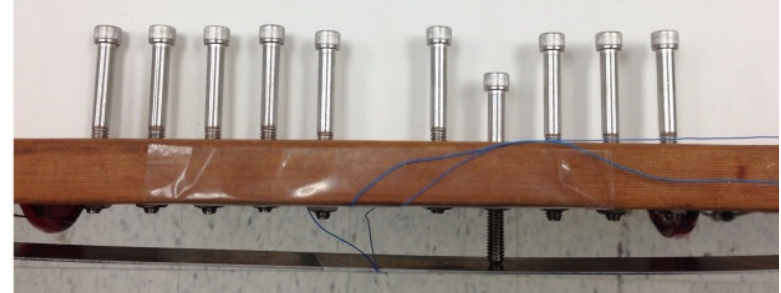

Figure 5-1(a) Frame (b) Screws to apply load to the beam 


\subsubsection{Results}

The effect of loading on the surface response of different points distributed to the beam was studied by using the scanning laser vibrometer. The vibrations of 72 points located at a $3 \times 24$ grid were monitored automatically. As it is shown in the Figure 5-2, the points on the piezo are deactivated meaning that laser vibrometer is not going to scan them. The reason is that the target structure to be scanned is the beam surface and points on the piezo do not reflect the surface vibration of beam. For scanning any target it should always considered to eliminate scan points on the piezo element because of high level of noise in those spectrums the post processing could lead to problematic results.

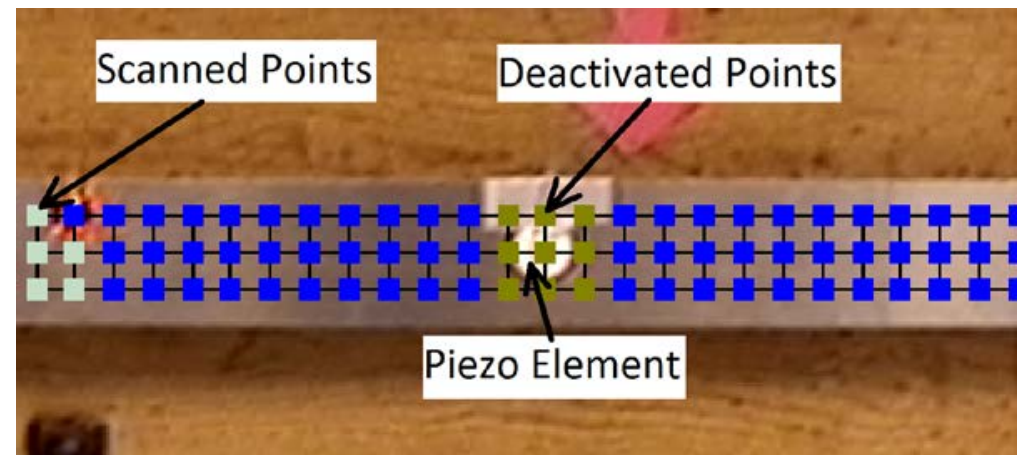

Figure 5-2 Scanning grid; blue is scan points, white is already scanned and brown is deactivated points

Figure 5-3 shows the results at three different loading conditions. In each case the hot spot close to the bolt that applied the force to the beam. The color map diagram shows the distribution of the normalized value of the sum of square of differences between the no load and loaded spectrums. A force was applied to the left side, center and right side of the beam. Similar results obtained were obtained when the tests were repeated with three beams with different thicknesses (1/8”, 1/16” and 1/32”). The thinner plate was affected the most since the applied torques to the bolts was the same in all 
experiments. The hot spot most accurately identified the loading location for the thinnest plate.
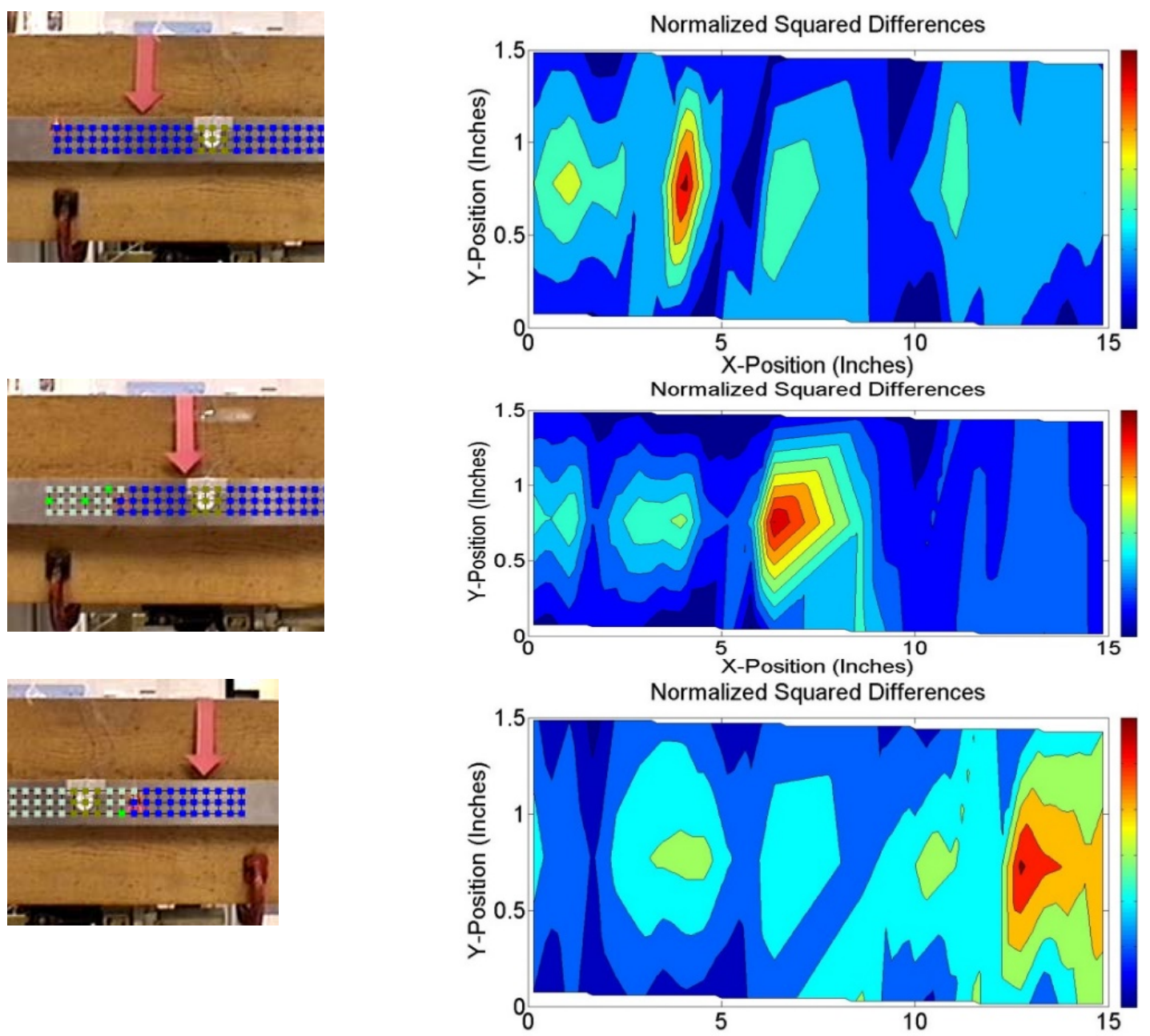

Figure 5-3 Location of application of force on the plate and the variation of normalized sum of the squares of the differences

\subsubsection{Conclusion}

In this study, the loading location estimation accuracy of the surface response to excitation (SuRE) method was studied. To evaluate the oscillations of the entire surface scanning laser vibrometer was used. The sum of the squares of the differences was calculated with respect to references of 72 points at a $3 \times 24$ grid. The study indicated 
that the scanning laser vibrometer may be used for implementing the SuRE method and the location of the applied force on a plate may be detected.

Based on these results, the SuRE method may be used for remote sensing of structural problems and loose components. Also in cases where the location is not accessible or economically cost effective to implement the laser scanning vibrometer, the defect or loading location may be estimated by using the SuRE method if multiple piezoelectric elements are used for sensing.

5.2. Inspecting Integrity of a Multi-Bolt Robotic Arm Using a Scanning Laser Vibrometer and Implementing the Surface Response to Excitation Method (SuRE)

\subsubsection{Introduction}

The integrity of a robotic arm was examined remotely via a scanning laser vibrometer (SLV) in order to detect loose bolts. A piezoelectric element (PZT) was bonded on the robot arm for exciting surface guided waves. A spectrum analyzer generated surface waves within the $20-100 \mathrm{kHz}$ range. The propagation of the waves was monitored with the SLV at the programmed grid points on the robot arm.

The surface response to excitation (SuRE) method was used to calculate the spectrums of the signals, and compare the reference scan with the altered scan. Comparisons of before and after the scan showed that after loosening the bolt on the robot arm, spectrums of all the grid points changed to some extent, however, the largest changes occurred in the vicinity of the loosened bolts.

The study shows that the SuRE method was capable of detecting the presence and location of loosening bolts using only one PZT element on a complex structure. There are 
two most important advantages of the SuRE method over the widely used impedancebased technique. The first advantage is the elimination of an expensive impedance analyzer; the second advantage is remotely monitoring capability as long as the surface is excited properly.

\subsubsection{Experimental setup}

The surface vibrations were measured at the programmed grid points by using the PolyTech PSV-400 SLV. The Stanford Research Systems SR780 2-channel network signal analyzer generated the excitation signal and monitored the signals coming from the SLV. A power amplifier magnified the signal of the signal analyzer before it was given to the PZT. (Figure 5-4)

The signal analyzer calculated the spectrum after the signal was digitized. The analog sensors of the laser head itself can monitor the vibrations well above $100 \mathrm{kHz}$, but the vibrometer controller analog-to-digital converter sampling frequency peaks at 100 $\mathrm{kHz}$. This caps the maximum frequency that the laser software can scan at $40 \mathrm{kHz}$. To overcome this limitation, the Stanford signal analyzer with a maximum A/D conversion rate of $250 \mathrm{kHz}$ was connected to the laser head. This allows us to capture frequencies up to $100 \mathrm{kHz}$. 


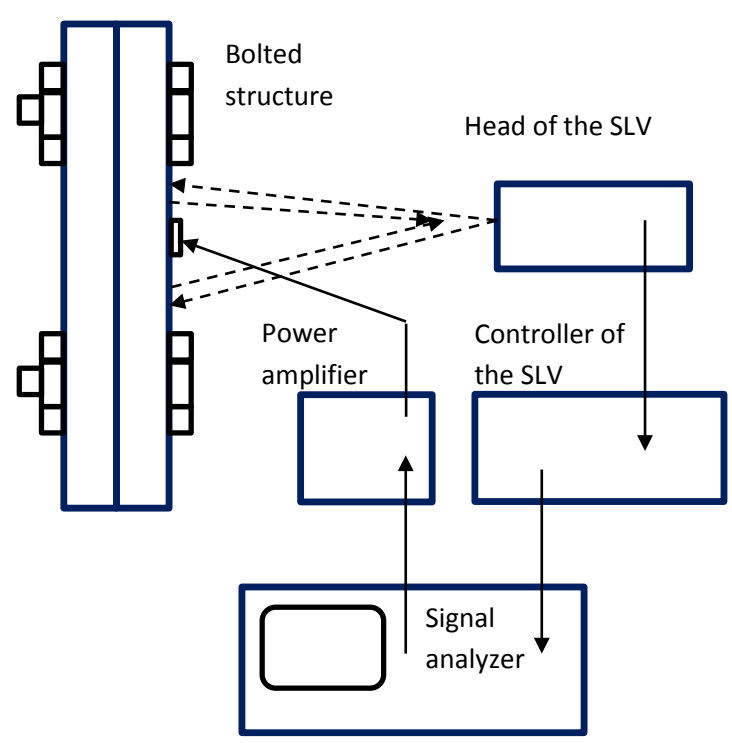

Figure 5-4 Experimental schematic

The laser system can operate for long periods of time and conduct scans at multiple points in a relatively short period of time. The signal analyzer can only scan one point at a time. During the experiment, the operator manually selected points with the laser and performed scans with the signal analyzer. The low and high ends of the sweep sine wave signal were selected as $20 \mathrm{kHz}$ and $100 \mathrm{kHz}$, respectively. The low end was selected to prevent audible sounds in the lab. The maximum sampling frequency of the signal analyzer's A/D determined the high end.

The dimensions of the aluminum robotic control arm were 152.5(cm)×47(cm)×33(cm) (Figure 5-5). A 3/4” diameter APC PZT model D-.750"2MHz-850 WFB was attached to the center of the scanned area where the bolts were located. The dimensions of the scanned plate were: $78.75(\mathrm{~cm}) \times 7.62(\mathrm{~cm})$ with a $1.27(\mathrm{~cm})$ thickness and the bolts were located approximately $7.62(\mathrm{~cm}) \times 4.45(\mathrm{~cm})$ apart from each other. 18 bolts were used for the structure. 


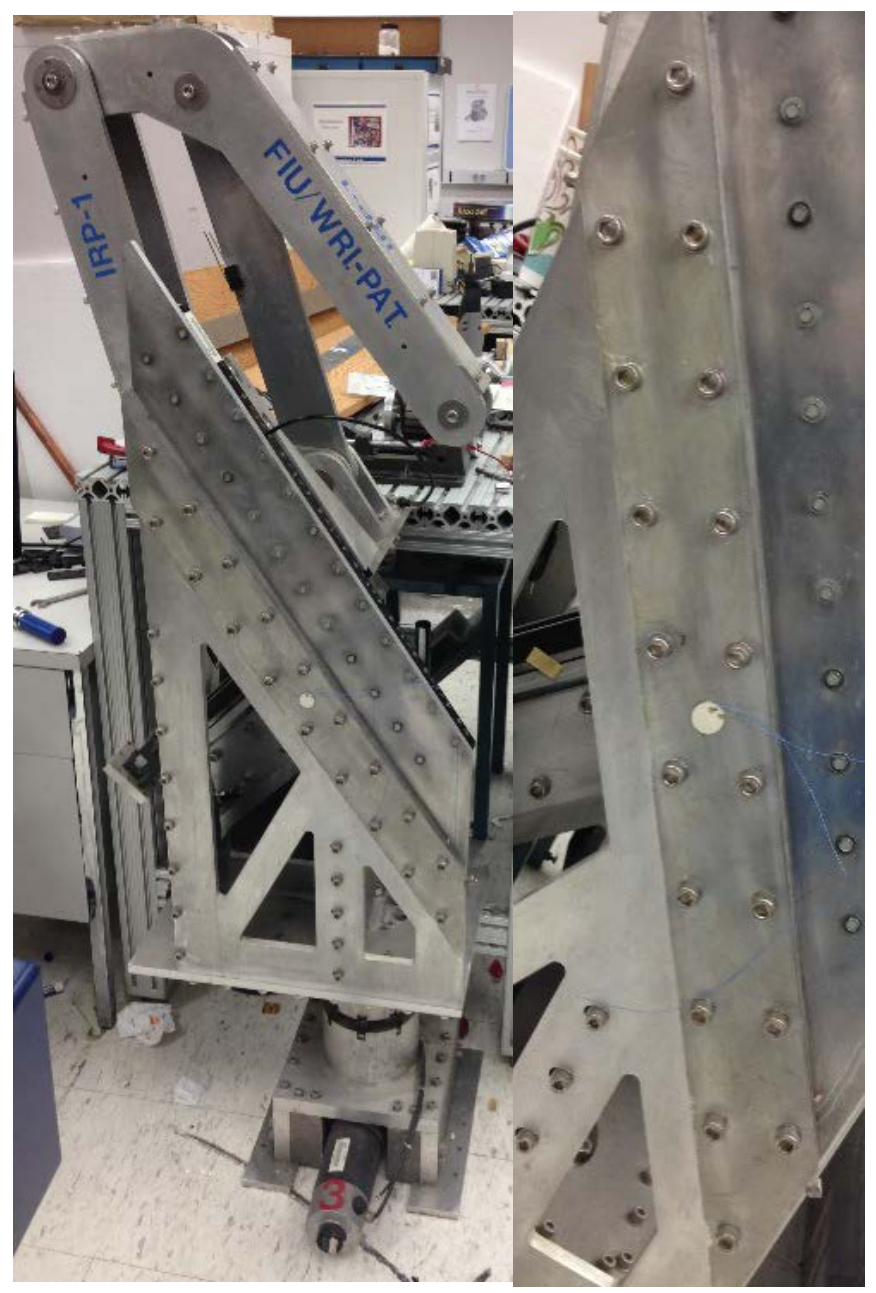

Figure 5-5 The robot structure and close up view of the scanned section

The SLV was located five feet away from the control arm of the robot. The reference data was collected when all bolts were tight by scanning the vibration at the points on the grid. Then, one bolt or multiple bolts were loosened and another scan (altered data) was measured using the same grid points. Finally, these scans were compared to each other using the algorithm described in the theoretical background. The 
positions of loose bolts were identified by locating the scan points with the highest NSSD values.

\subsubsection{Results}

The tests were performed on a robot arm with a bolted joint that was connected to the main structure via 18 bolts and nuts, out of which 16 were considered for this study (Figure 5-6).

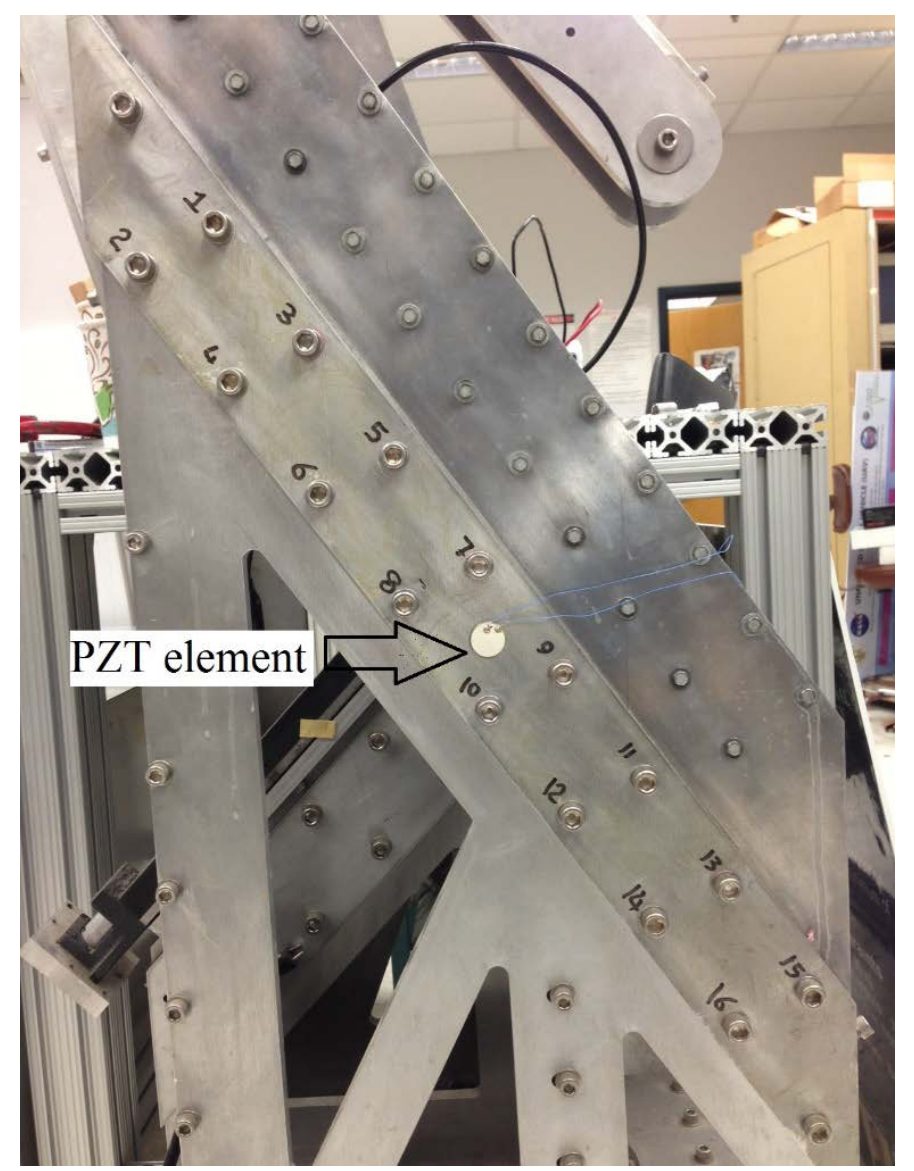

Figure 5-6 Bolted joint on the robot arm with bolt numbers 


\subsubsection{Natural Frequencies}

The simple bump test method [65] was used to determine the natural frequencies of the robot arm. The structure was hit with an impact hammer and the signal was collected from the PZT element on the arm. The Fast Fourier Transform (FFT) of the signal was calculated. The dominant peaks, which were determined by peak hold of the instant FFT response, were used to determine the natural frequencies of the structure. Table 1 shows the first five natural frequencies of the robot arm in two separate tests of the tight and loose bolt cases:

Table 5-1 Natural frequencies of the robot arm

\begin{tabular}{lrrrrr}
\hline & $1^{\text {st }}$ & $2^{\text {nd }}$ & $3^{\text {rd }}$ & $4^{\text {th }}$ & $5^{\text {th }}$ \\
\hline Tight Bolt & $61.35(\mathrm{~Hz})$ & $82.39(\mathrm{~Hz})$ & $180.04(\mathrm{~Hz})$ & $381.46(\mathrm{~Hz})$ & $628.61(\mathrm{~Hz})$ \\
& & & & & \\
\hline Loose Bolt & $61.35(\mathrm{~Hz})$ & $82.39(\mathrm{~Hz})$ & $180.05(\mathrm{~Hz})$ & $381.47(\mathrm{~Hz})$ & $628.62(\mathrm{~Hz})$ \\
\hline
\end{tabular}

To test the effect of the loose bolts on the natural frequencies of the structure, the measurements were repeated after loosening the single and multiple bolts. The changes of the natural frequencies were very small. In the experimental studies, such small changes have been observed when the experiments were repeated at the same conditions. Environmental noise and small computational errors of signal analyzers could cause those changes. Our results agreed with the previous studies like Todd et al. (Todd, Nichols, Nichols and Virgin 2004), where the natural frequencies did not exhibit enough sensitivity detecting loose bolts. 
In our study, high frequency surface waves were employed and the changes in the measured spectrums were used for detecting the single and multi-loose bolts similar to the impedance method.

\subsubsection{Detection of Loose Bolts in Linear Grid}

The first experiment was designed to identify the effect of the presence of a single loose bolt on the measured spectrum. First, a single scanning point was chosen near the location of bolt 10. The initial reference scan was measured when all the bolts were tight. The reference scan measurement was repeated before loosening any bolt to evaluate the consistency of test characteristics. The altered scan data was collected for after the bolt was loosened.

The spectrums resulting from the first two scans were almost overlapping each other over the whole frequency range 20-100 kHz. This can be observed in Figure 5-7 (a). This observation demonstrated consistency of the data collection and analysis system. The third spectrum was very different than the first two, Figure 5-7 (b). The propagation characteristic of the surface waves was consistent as long as the bolt was tightened. The propagation characteristic drastically changed when the bolt was loosened. The impedance method detects the presence of loose bolts by monitoring the same characteristics of the impedance spectrum. 
(a)
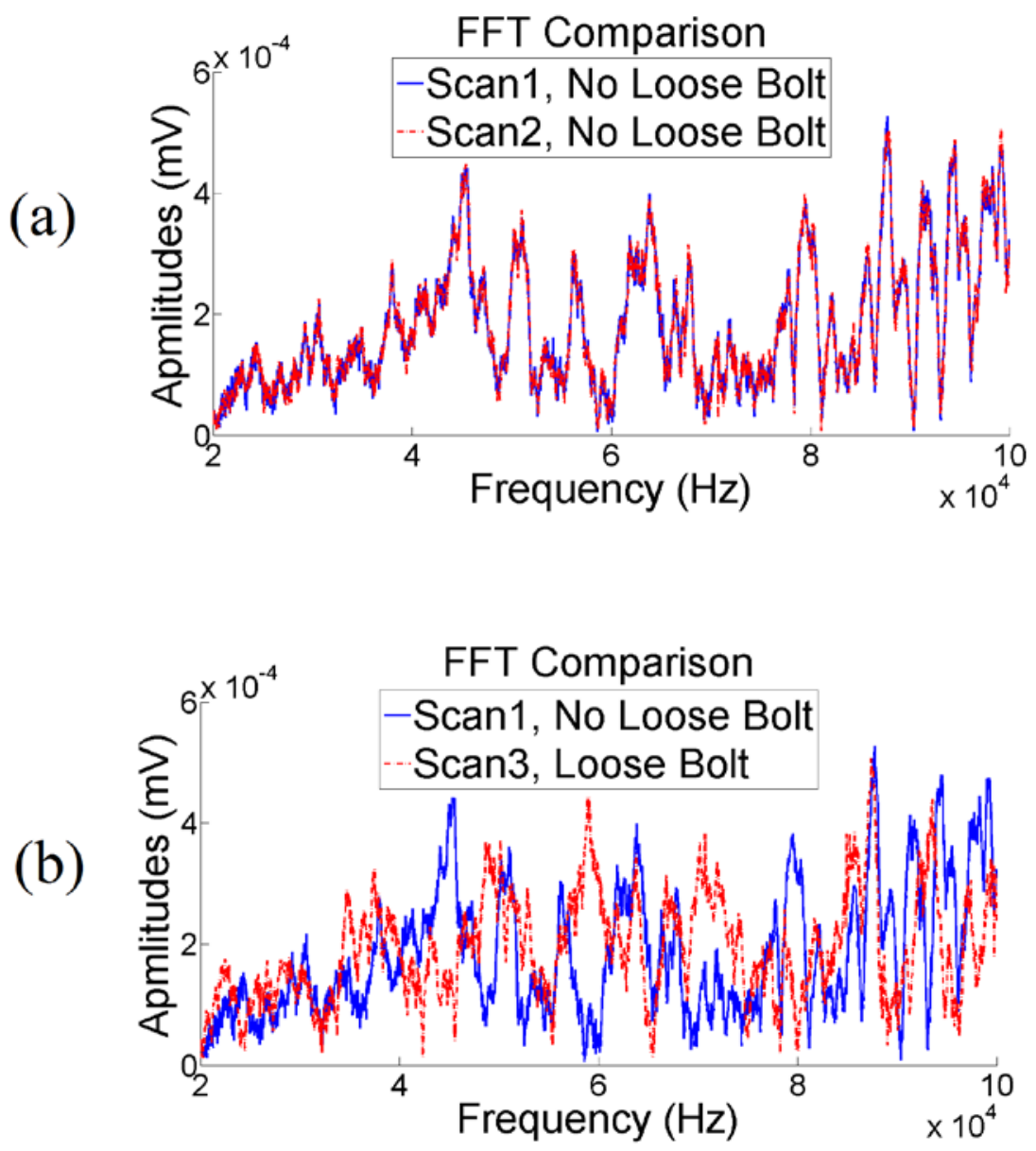

Figure 5-7 FFT spectrum comparison (a) Scan1 with all bolts tight and Scan 2 with all bolts tight (b) Scan1 with all bolts tight and Scan 3 with a loose bolt

The next scan was performed over a linear grid including four bolts in a row. The bolts 4, 6, 8 and 10 in Figure 5-6 were considered. After the reference scans, only bolt 8 was loosened. For each bolt, two scanning points were specified on the grid. These points were located on the left and right-hand side of the bolts (Figure 5-8). 


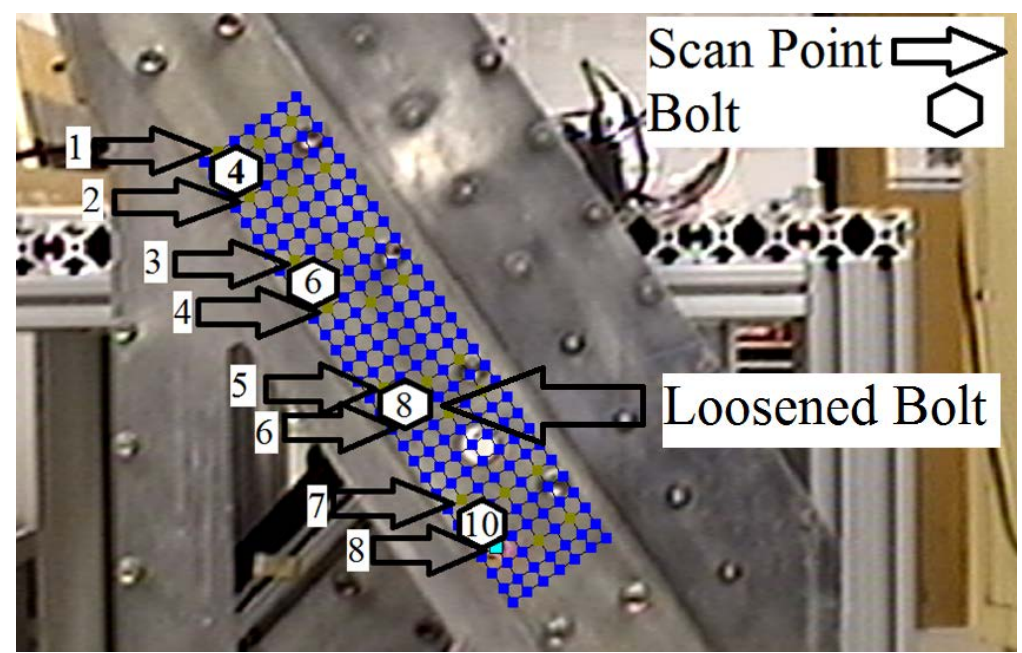

Figure 5-8 Local linear scan grid with eight scanning points specified on both sides of the lower four bolts.

Based on the algorithm developed on the theory section of this paper, the normalized sum of squared differences (NSSD) was calculated from the spectrums before and after loosening bolt 8, which was located between scan points number 5 and 6 in Figure 5-8. Figure 5-9 compares the NSSDs for bolt 8 when it was tight versus loose. Figure 5-9 (b) was used for estimating the location of the loose bolt. The NSSD of the scanning points 5 and 6 were very high compared to the other scan points. Since the scan points 5 and 6 are located by bolt 8 , this result clearly indicates that bolt 8 was loosened. The results of this section also confirm that the highest values of the NSSDs are observed at the nearest scanning points to the loosened bolt. 

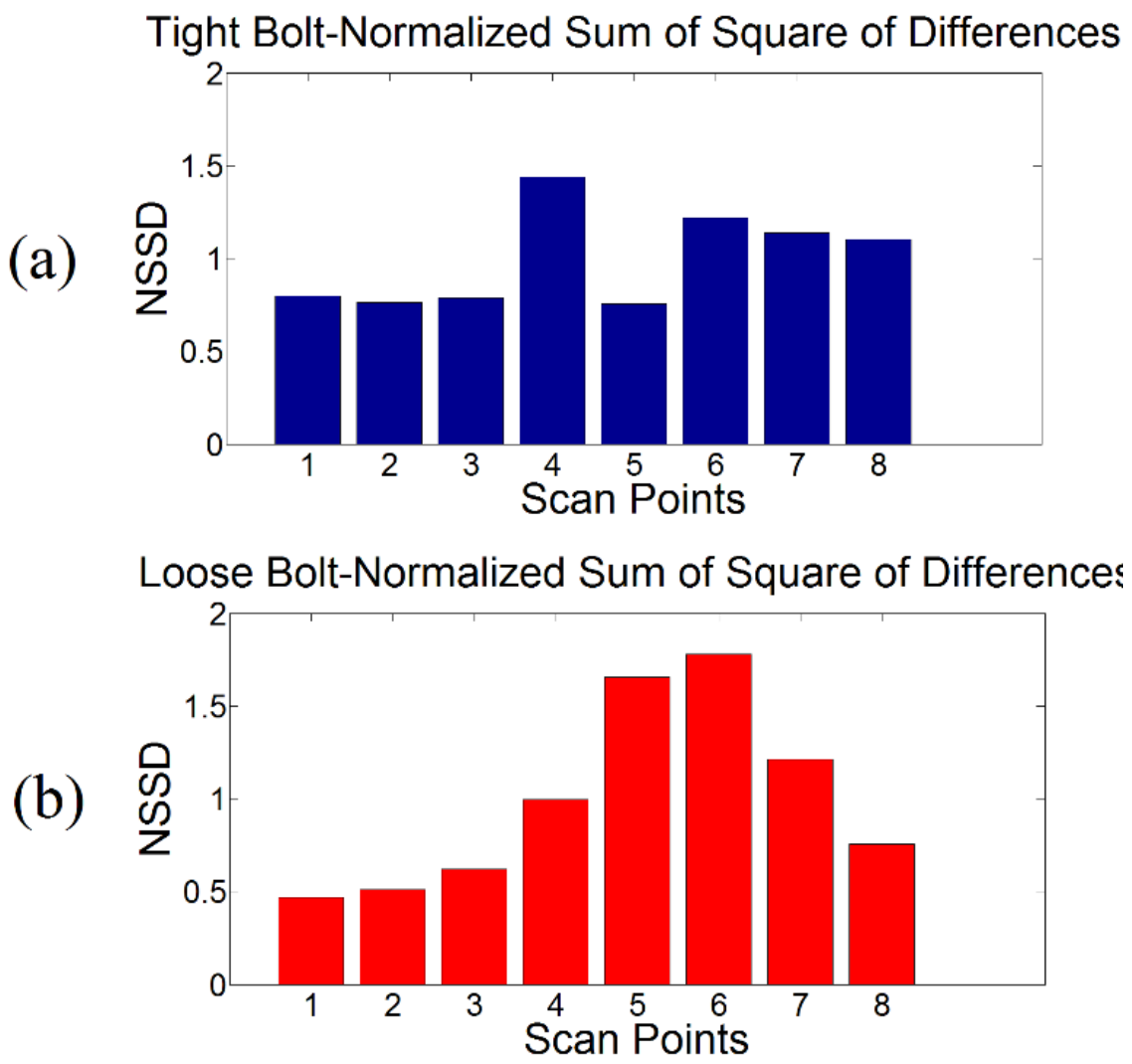

Figure 5-9 Normalized sum of square of differences for linear local scan for (a) before (b) after; single bolt was loosened

The typical changes of the SSD values of a structure without any loose bolts and with one loose bolt are presented in Figure 5-10. The SSD values of the scan points are due to measurement errors, external noise and calculation errors. These values were close to each other when there were no loose bolts. The SSD values of all scan points increased more than $70 \%$ when a bolt loosened. A threshold value may be found easily since the change was significant. The change of the SSD at the scan point closest to the loose bolt was more than $150 \%$. Based on this figure, the SSD values may be used for detecting the loose bolt and estimating its location. Using the normalized SSD (NSSD) values are more convenient for locating a loose bolt while the existence of the problem is detected from the SSD values. 


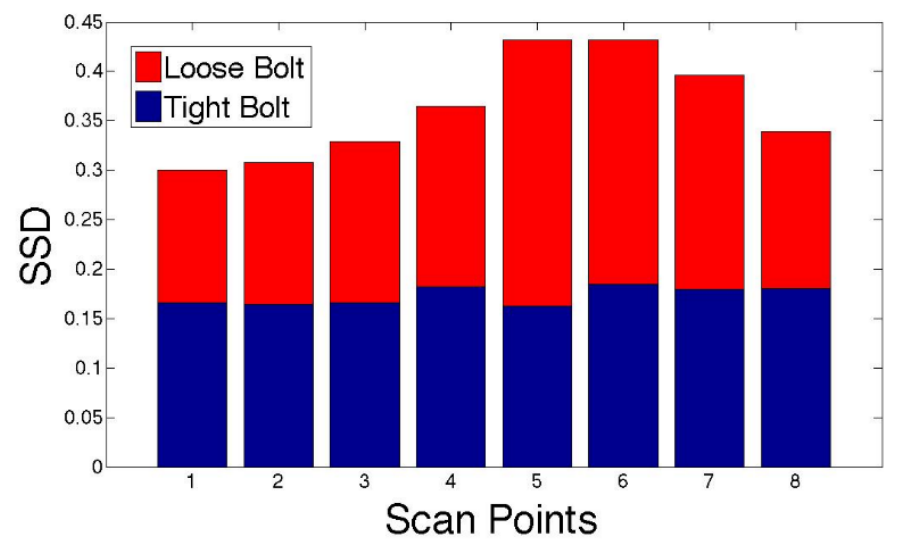

Figure 5-10 Sum of Squared Difference (SSD) values before and after loosening bolt 8 .

The proposed method may be implemented in the field by using the following procedure: First, the SSD values of the scan points are calculated. If the SSD values are below a threshold, the structure should not have any loose bolts. To improve the robustness of the method, the average of the SSD values are calculated and a secondary threshold is established by multiplying the average SSD value with a coefficient such as 1.2. The secondary threshold may be compared with the SSD values of the scan points to avoid false alarms if the diagnostic system is used in extremely different operating conditions. Such as testing the system when the engines are at the idle and cruise speeds. The second step will be used when some of the SSD values are over the threshold. The location of the loose bolt will be estimated by using the NSSD values.

\subsubsection{Detection of Loose Bolts in Two Dimensional Grid}

The two-dimensional grid was designed to locate one scan point next to each bolt. Therefore, the number of scanned points and the number of bolts on the structure were the same. The reference scan and a second scan with the same conditions were performed to confirm the consistency of the results. 
Figure 5-11 shows the normalized sum of squared differences when both scans were measured before loosening the bolt (first and second reference scans). There are 16 bars and each one corresponds to a point close to one of the bolts

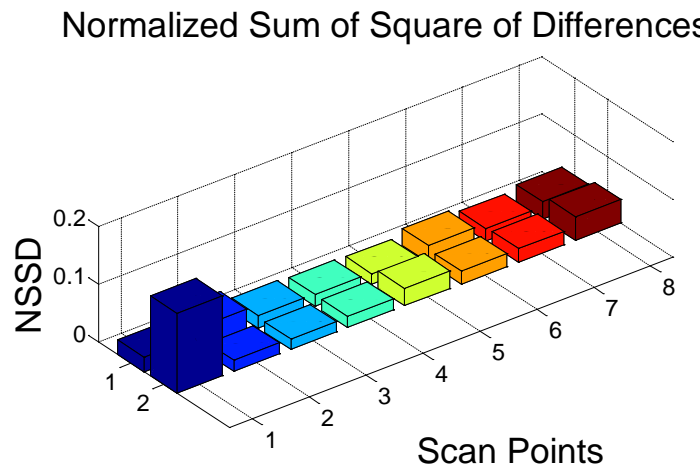

Figure 5-11 Bar diagram of normalized sum of square of differences for full arm without any loose bolts

In Figure 5-11 no major changes were identified for scan points before loosening the bolt. Another scan was performed after loosening two bolts in the upper row (bolts 3 and 5). Figure 5-12 shows that the approximate location of these bolts could be detected.

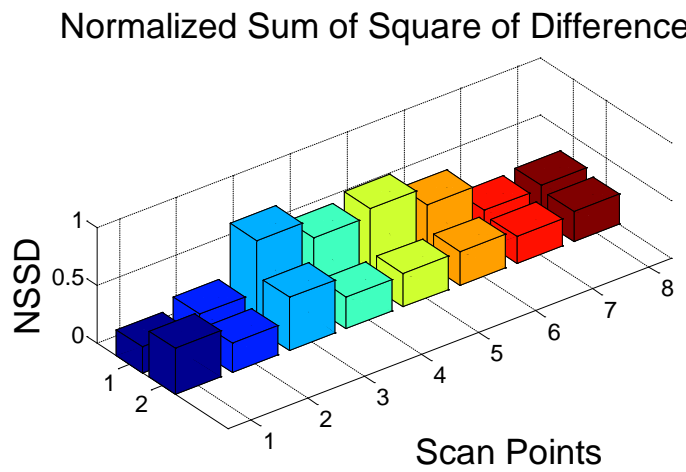

Figure 5-12 Bar diagram of normalized sum of squared differences for full arm with two loose bolts

The test was repeated after the bolts close to the scanning points 3 and 5 were loosened. The contour map of Figure 5-13 shows that the highest peaks are at the 
scanning points close to the location of the loose bolts. The adjacent areas were also affected. The contour map shows that the highest peaks were located at columns 3 and 5 , and identified the loose bolts better. The contour map was not smooth since the numbers of the grid points were very limited.

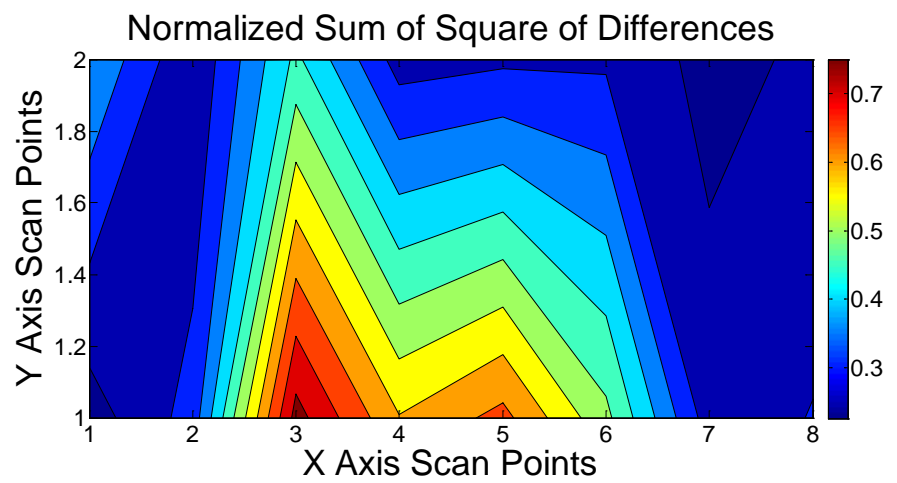

Figure 5-13 Contour map of normalized sum of squared differences for full arm scans with two loose bolts; bolt 3 and bolt 5 are loosened.

\subsubsection{Detection of Loose Bolts in Full Arm Grid}

In this section, an experiment for a single loose bolt was performed, followed by another experiment using multiple loose bolts. The density and size of the scan grid were designed to cover the whole bolted section of the robot arm.

The size of the scan grid was increased to improve the resolution and to demonstrate the consistency of the results. The vibrations of 60 points were monitored on a $20 \times 3$ scan grid (Figure 5-14). 


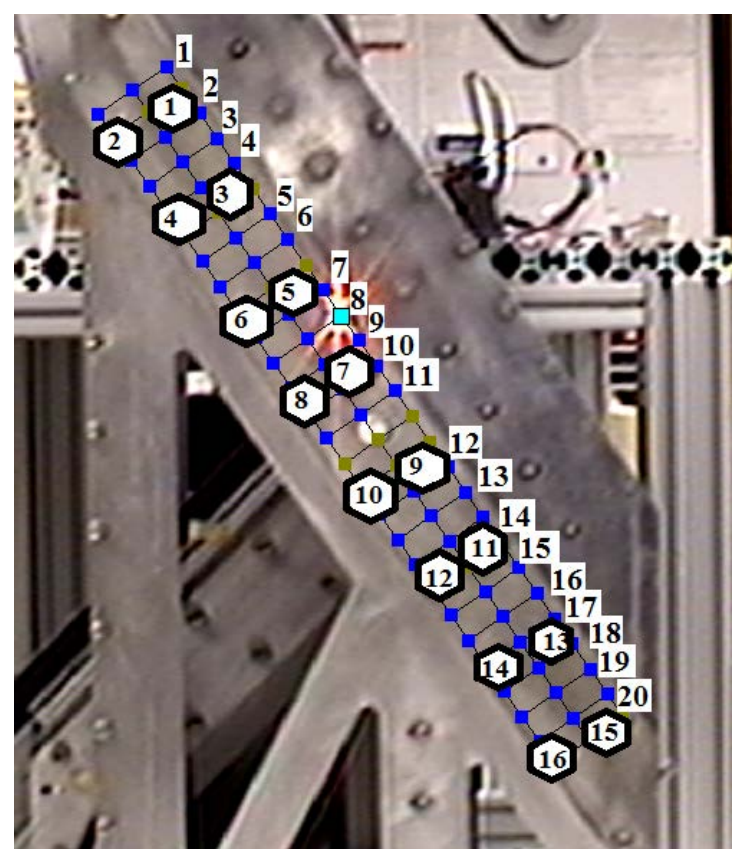

Figure 5-14 Two-dimensional full arm scan grid with 20 columns and 3 scan points in each column

Data was not collected from the scan points that were located on the piezoelectric actuator. The scan test points are illustrated with the blue points. Figure 5-15 shows a three-dimensional bar diagram that demonstrates the normalized sum of the squared differences. In this experiment, bolt 8 was loosened.

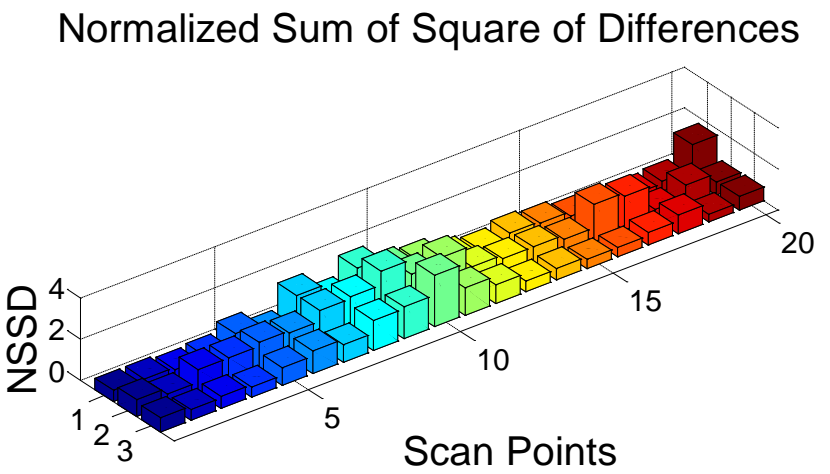

Figure 5-15 Normalized sum of squared differences for full arm scan; only bolt 8 is loosened 
The maximum values of NSSDs are located around columns 9 and 10 in Figure 5-15. In Figure 5-14), columns 9 and 10 are encircling bolt 8 . This means that the location of the maximum NSSD values correctly identified the loosened bolt 8 .

The two-dimensional contour map in Figure 5-16 is also prepared using the same NSSD values, where the location of the loose bolt is better identified.

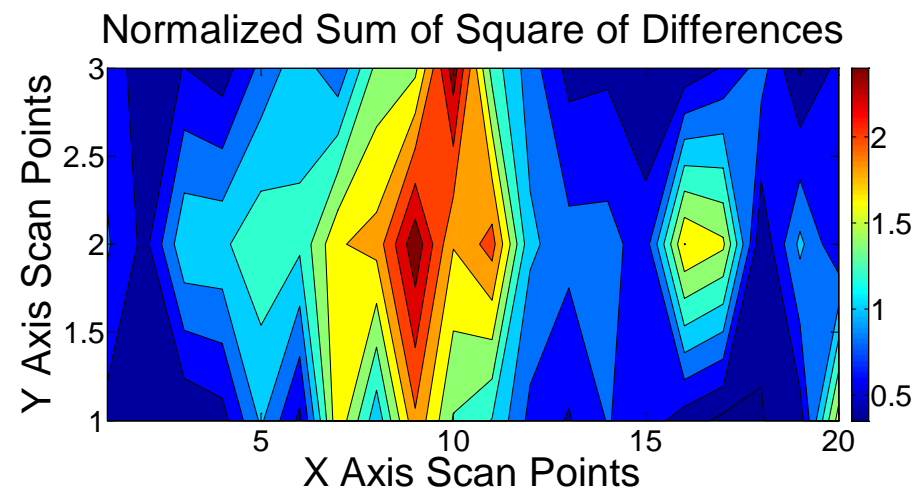

Figure 5-16 Contour map of normalized sum of squared differences for loosening bolt 8

The red spot in Figure 5-16 shows the peak of the NSSD values that corresponds to the scan points 9 and10.

\subsubsection{Detection of Multiple Loose Bolts in Full Arm Grid}

The reference data was collected when all bolts were tightened. The same $20 \times 3$ scan grid in Figure 5-14 was used in this experiment. To evaluate the performance of the proposed approach in a more challenging scenario, bolts 2 and 7 located at opposite corners of left side of the scan grid were loosened. The normalized sums of squared differences were calculated and those values were used to prepare the 3D bar graph and 2D contour map. 


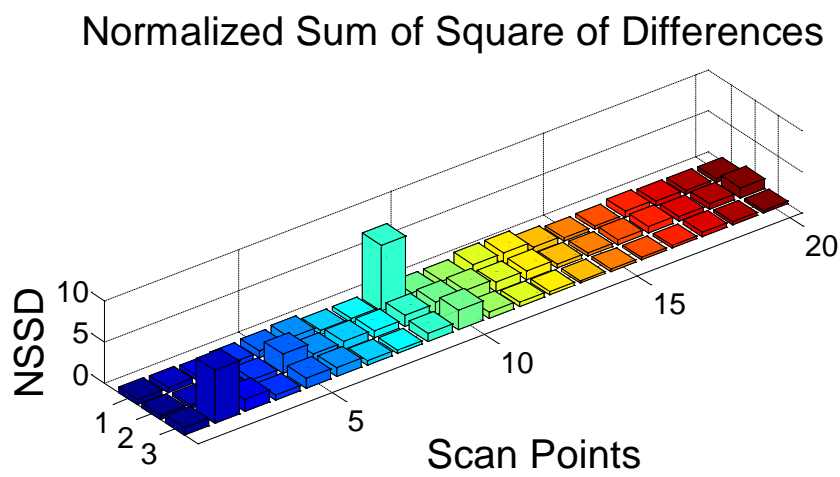

Figure 5-17 Normalized sum of squared differences for full arm scan; bolt 2 and bolt 7 are loosened

Figure 5-17 shows the NSSD values when bolts 2 and 7 were loosened. The highest peaks in this bar graph were located at the lower 2nd column and upper 9th column of the scan grid. These scan points are adjacent to the bolts 2 and 7 in Figure 5-14.

The contour map of the NSSD values presented in Figure 5-18 clearly illustrates the location of the loose bolts within two red spots.

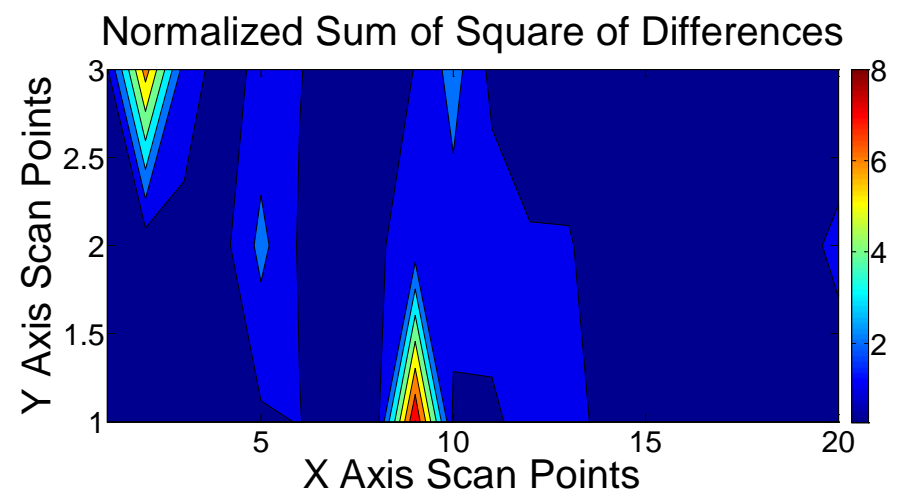

Figure 5-18 Contour map of the normalized sum of square of differences for multiple bolt loosening

The spectrums of each point were very similar when the test was performed using the same test conditions. When one or multiple bolts were loosened the propagation characteristics of the surface waves were changed. The changes of the spectrums were 
consistently more significant around the loosened bolts. The location of the loose bolts could be easily visualized with the help of 3-D bar graphs and 2-D contour maps. The results improved by increasing the grid points. The optimal grid size depends on the geometry and material of the structure, and may be determined with several experiments.

Here, it is noteworthy to emphasize the importance of attention to the noise problem during the experiments. Since the signal analyzer uses the peak hold to capture the frequency spectrum, large oscillations in the input signal during the capturing process could influence the peak holds and change their original values. During the data acquisition process, operator must constantly monitor the spectrum on the signal analyzer screen. In the case of any out-of-focus operation, the level of noise in the spectrum increases instantly and eats up the lower parts of the frequency spectrum throughout the entire frequency spectrum (Figure 5-19). Once the spectrum is distorted due to noise, it will no longer overlap the intact frequency spectrum from the same scan point. This way the comparison algorithm of SuRE method will calculate an increased NSSD value for that point and system will generate a false alarm.

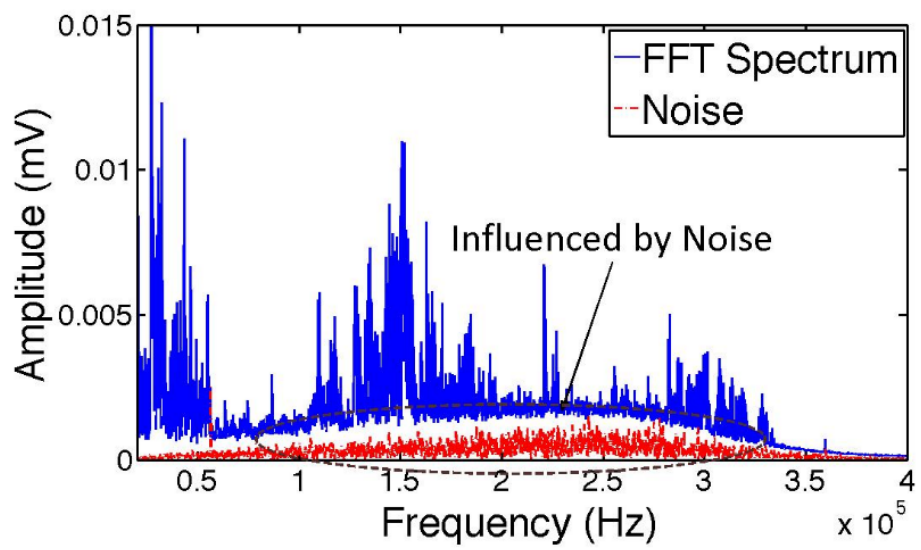

Figure 5-19 Effect of noise in the FFT spectrum 


\subsubsection{Conclusion}

The surface response to the excitation (SuRE) method was used to identify the loose bolts on a large robot structure. The surface was excited with a piezoelectric element and the response was monitored remotely with a scanning laser vibrometer.

The loose bolts were identified accurately at all tests; either single bolt or multiple bolts were loosened. The surface vibrations were very small and required careful data collection with a separate spectrum analyzer. However, the SuRE method was found effective for detecting the loose bolts and identifying their location. The scanning laser vibrometer was able to collect the data very quickly and test many points on the structure without attaching a large number of piezoelectric elements at the considered grid location. In addition, the scanned surface area under the laser beam was extremely small and convenient for special applications involving miniature machine components.

The study was performed with different scan grid sizes to investigate the effect of the grid size in the results. It was necessary to have at least one scanning point next to each bolt to be able to locate the loose bolt(s). The scan grid with the minimum grid points required the minimum scanning time. Increasing the number of the grid points proportionally increased the scanning time while the resolution improved, and consequently the locations of the loose bolts were identified more accurately. The 3-D bar graphs and 2-D contour maps helped visualizing of the location of the loose bolts. Among them, 2-D contour maps were more effective when the number of grid points were satisfactory. 
The major challenge of this study was the high noise to signal ratio. The small amplitudes of the surface waves and remote measurement of these tiny surface oscillations were two primary sources of the noise. The surface waves were created with a PZT element attached to the surface. To maximize the amplitude of the surface waves, a power amplifier was used to provide higher power to the PZT element than the spectrum analyzer can provide. However, at the $20-100 \mathrm{kHz}$ frequency range, the amplitudes of available exciters are much smaller compared to the typical electrodynamic exciters operating at the $0-200 \mathrm{~Hz}$ frequency range. In addition, the amplitude of the waves decreases while the waves travel from the PZT to the boundaries.

The second source of noise was from scanning from a remote location to measure the tiny surface waves. Compared to monitoring the voltage coming from the PZTs attached to the surface, the noise is much higher at the SLV outputs. This could be due to the noncontact nature of the measurement and very small scanning surface area. In addition, the power of the laser beam was lower at the boundaries of the scanned grid compared to the central point. The auto focusing capability of the SLV avoided out-offocus operating the optic system at the boundaries. The size of the scan area should be carefully selected to be sure that the noise is within the acceptable levels when the boundaries are scanned. Therefore, there is a limitation in the dimension of the scan area depending on the distance of the laser head from the target area.

In practical applications, assessment of the environmental noise is recommended before implementing any SHM method. Generally, forced vibrations and their harmonics disappear within $20 \mathrm{kHz}$; however, impact forces are felt throughout the entire spectrum. If the environmental noise is very low at the test frequency range, the SuRE method may 
be implemented by using the SLV. Instead of the SLV, multiple piezoelectric elements may be attached to the grid points for implementing the SuRE method if the noise is too high for the SLV system. Authors believe the noise problem needs to be thoroughly investigated before implementing the proposed method at the field.

\subsection{Surface Response to Excitation Method for Structural Health Monitoring Using} Neural Networks

\subsubsection{Introduction}

Composite materials have been preferred for critical applications when maximum strength/weight ratio and corrosion resistance are required. In addition, composite materials with embedded sensors have been considered for aerospace vehicles with powerful structural health monitoring (SHM) capabilities. Piezoelectric materials have been widely used for SHM applications due to their dimension change capability with charge and low price [141].

Surface response to excitation (SuRE) method used separate piezoelectric elements for excitation and sensing to eliminate the need for impedance analyzer used by the electromechanical impedance technique. SuRE excited high frequency surface waves on the structure and directly measured the voltage from piezoelectric element(s). Both methods detected the damage of a structure or change of the loading conditions by calculating the sum of square of differences between the spectrums of the sensor at different stages of the life of the structure in most studies. The purpose of this study was to estimate the location of a compressive load applied to a composite glass-fiber/epoxy plate with minimal human input. Two piezoelectric elements were used for excitation 
and sensing. The spectrums of the received signals were classified by using neural networks.

\subsubsection{Experimental Setup}

The performance of the SuRE method was evaluated using a DSP-based device and neural networks. The implementation procedure of SuRE method for SHM of a beam with a DSP has been discussed in section 3.6.1.

Both the excitation and sensing were performed using APC piezoelectric model D-.750”-2MHz-850 WFB. Two PZT elements were bonded on the $6 \times 2 \times 1 / 12$ (in) composite beam 2.5 inches apart from each other (Figure 5-20) using LOCTITE Hysol Product E-30CL epoxy adhesive.

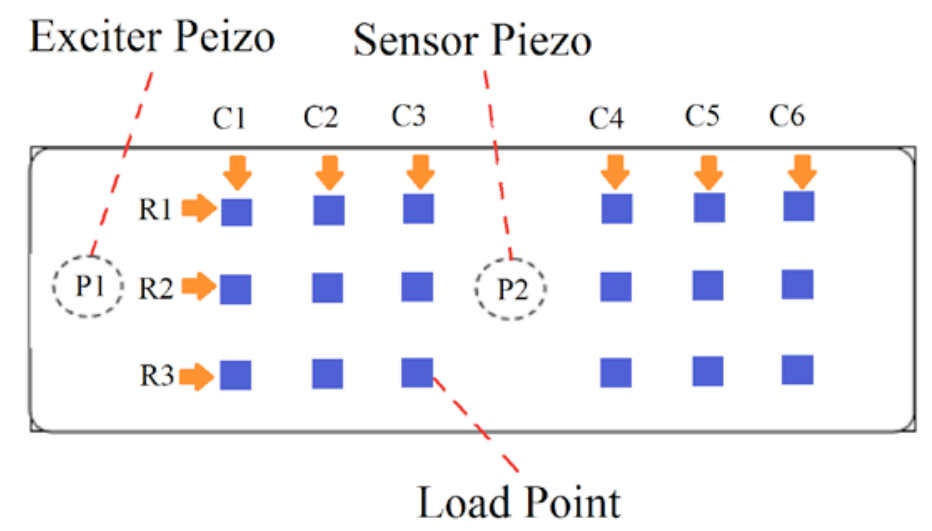

Figure 5-20 Load applied on four points (points 2-5) on a composite beam using a clamp

The excitation signal is a series of pure sine waves that starts with a frequency of $20 \mathrm{kHz}$ and goes up to $100 \mathrm{kHz}$ with changes of $100 \mathrm{~Hz}$ each $0.21 \mathrm{~ms}$ approximately (this time changes depending of the number of points desired to acquire for each frequency segment). The amplitude of these sine waves was always $6 \mathrm{~V}$. 
In this study, the spectrums were used for training two neural networks to detect the presence of load on an composite beam. Based on the SuRE method first a reference measurement was captured from a set of measurement points. The measurements were from piezoelectric elements and for training the neural networks $70 \%$ of the samples were used for training, $15 \%$ of the samples were used for validation and the last $15 \%$ of data was used for testing.

For each configuration, 20 experiments were taken and the spectrum saved. Total of 100 experiments were conducted. In the first approach for training, the LevenbergMarquardt algorithm trained neural network using this dataset a. the input of the neural network was 800 sample points that built the spectrum response. The output of it was a number between 1 and 6 depending of where the load was applied (Table 5-2). In the second approach for training, the data from the first row of load points in Figure 5-20 was used to train the neural network and the data from the second and third row were used to evaluate its performance.

Table 5-2 Output of neural network based on load condition

\begin{tabular}{lllllll}
\hline Number & 1 & 2 & 3 & 4 & 5 & 6 \\
\hline Load & Point 1 & Point 2 & Point 3 & Point 4 & Point 5 & Point 6 \\
\hline
\end{tabular}

For the Based on the method described in section 3.5.1, the SSD values were calculated for the five point loads. The behavior of SSD values after introducing the load were analyzed and compared to the performance of neural networks. 


\subsubsection{Results}

The signal received is converted to the frequency domain, taking for each frequency segment n_max maximum points and calculating the median point of this array. The parameter $n \_$max depends of the quantity of samples acquired per each frequency segment. A typical spectrum response is shown in Figure 5-21.

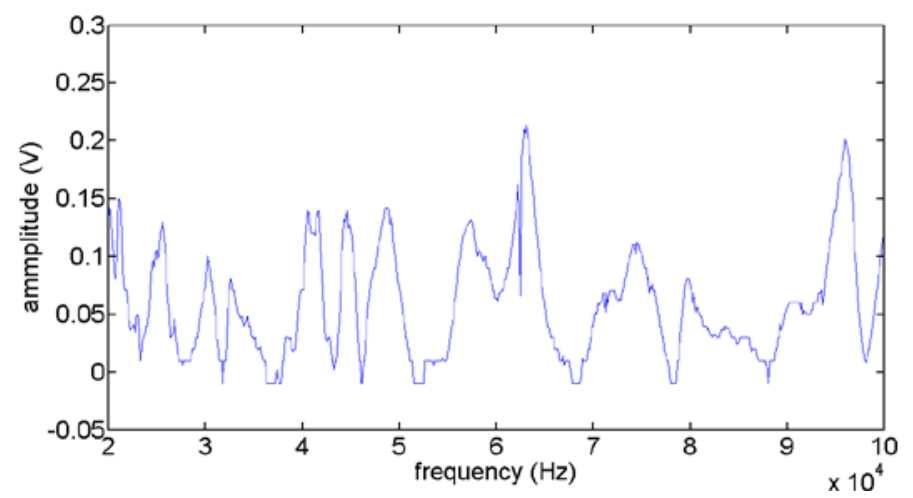

Figure 5-21 A typical spectrum built with the time domain data

Figure 5-22 compares the spectrums from signal generated while the load was applied to any of the 6 load points versus the original spectrum without load.

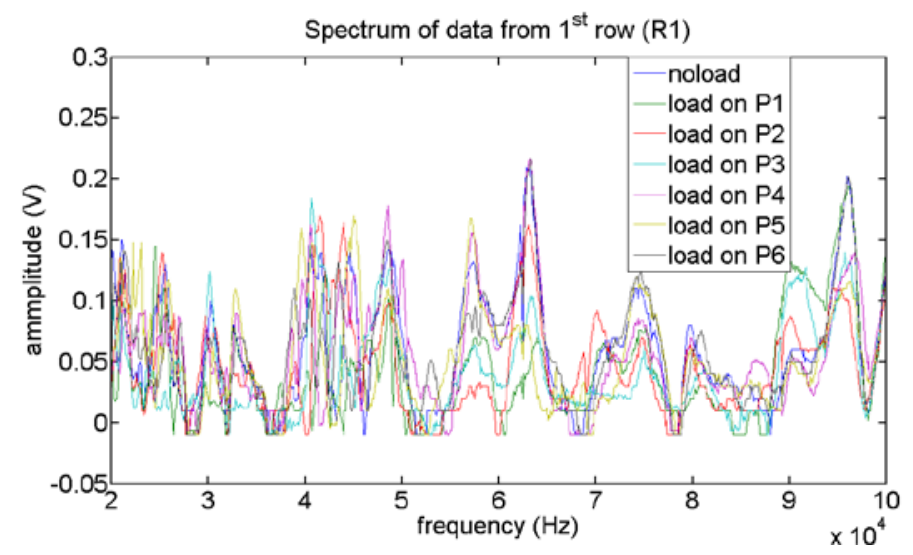

Figure 5-22 Comparison between spectrums depending of load application point 
Table 5-3 summarizes the average error and the average time spent for training of each algorithm. Using the scaled conjugate method, the results are highly accurate. Also, the spent time in training the algorithm is shorter than the Levenberg-Marquardt algorithm. Furthermore, the number of neurons at the hidden layer does not have any relation with the error. The average time spent for training the algorithm has a proportional relationship with the number of neurons used. The ideal number of neurons to be used for training the neural network is 12 .

Table 5-3 Result comparison between both training algorithms

\begin{tabular}{|c|c|c|c|c|}
\hline \multirow{2}{*}{$\begin{array}{c}\text { Neurons } \\
\text { at Hidden layer }\end{array}$} & \multicolumn{2}{|c|}{ Average Error [\%] } & \multicolumn{2}{c|}{ Average Time Spent [s] } \\
\cline { 2 - 5 } & Levenberg - & Scaled Conjugate \\
Marquardt & Gradient & Levenberg & Scaled Conjugate \\
Marquardt & Gradient \\
\hline 6 & 11.24 & 2.83 & 1.09 & 0.48 \\
\hline 9 & 16.87 & 1.84 & 1.17 & 0.42 \\
\hline 12 & 5.25 & 0.34 & 1.87 & 0.45 \\
\hline 15 & 7.53 & 8.72 & 2.98 & 0.39 \\
\hline 18 & 5.90 & 1.84 & 4.87 & 0.55 \\
\hline 21 & 13.28 & 0.81 & 6.04 & 0.59 \\
\hline 24 & 6.79 & 1.69 & 9.13 & 0.62 \\
\hline 27 & 5.75 & 0.03 & 10.47 & 0.54 \\
\hline 30 & 9.30 & 7.67 & 12.39 & 0.42 \\
\hline
\end{tabular}


Figure 5-23 shows the comparison of the classification results for one of the best performances of the best methods (i.e. with 12 neurons at the hidden layer). The estimations were very good in 27 of the 30 test cases.

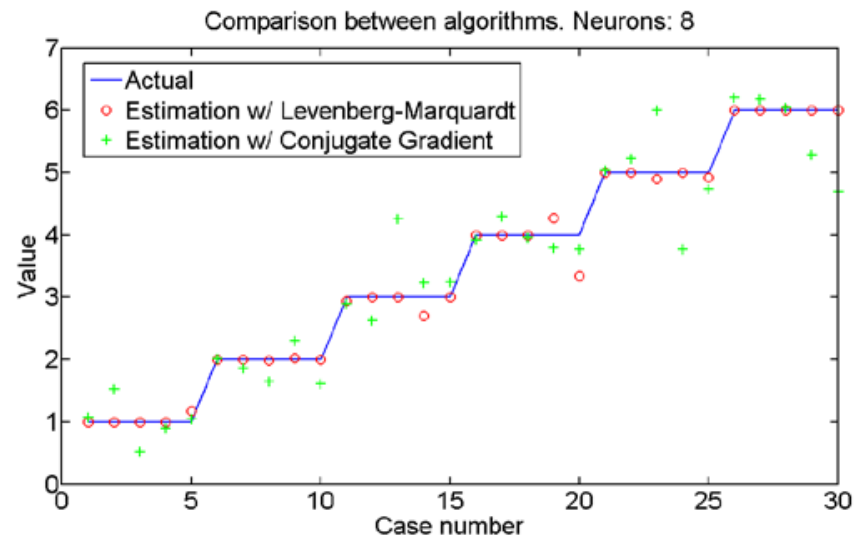

Figure 5-23 Comparison of classification results of both methods

The trained neural network was tested by using the spectrums when the load was applied to the second row. Separate neural networks were trained to estimate the column and row of the test points. The neural network estimated the test columns (Figure 5-24-a) of the applied load more accurately compared to the test row estimations (Figure 5-24-b). 

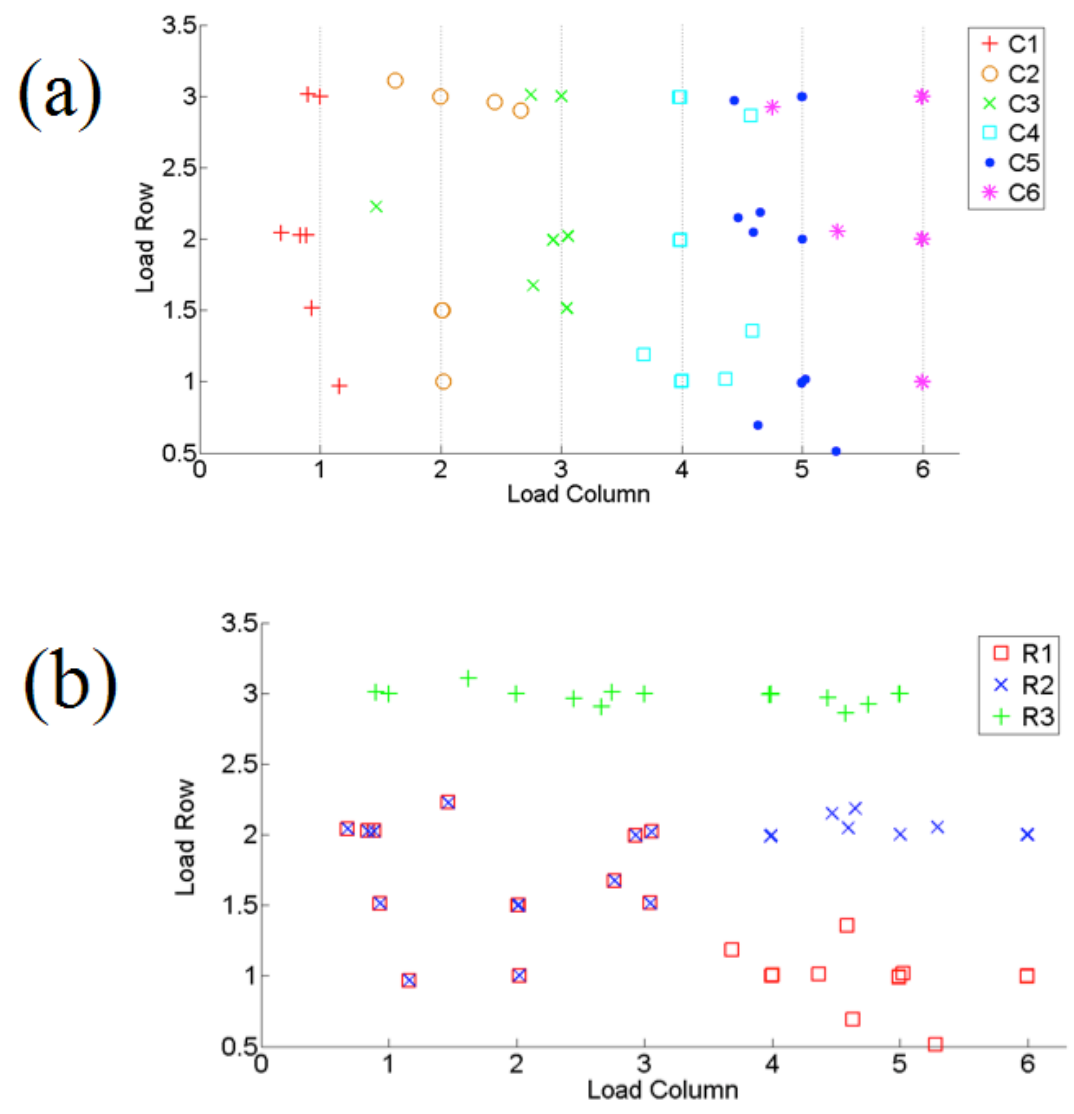

Figure 5-24 Performance of the neural network when (a) the test columns, (b) the test rows were estimated

The study indicated that the Levenberg-Marquardt type neural networks may be trained for estimating the location of the compressive forces on a composite plate by using the spectral characteristics. The proposed method is very effective for estimation of the distance of the compressed force from the end of the beam.

Figure 5-25 shows that sum of the SSD have been able to detect the presence of load on the beam. A jump in SSD values has occurred due to the presence of load on the beam. However, no consistent trend was observed as the load moved from point 2 to point 5. 


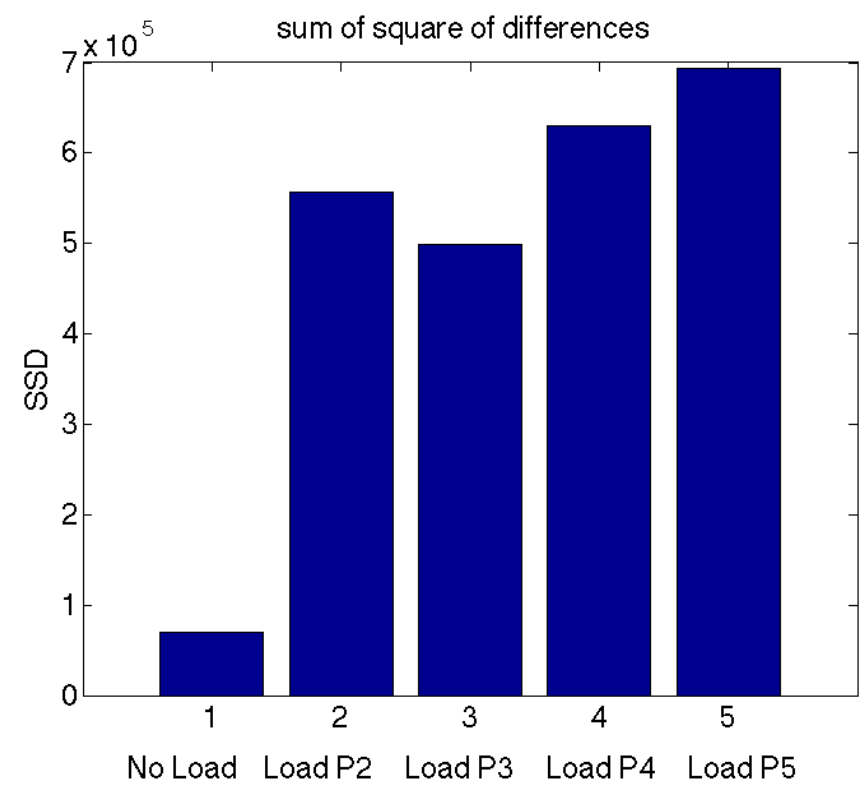

Figure 5-25 Sum of square of differences between the no load reference spectrum and load spectrums

\subsubsection{Conclusion}

The implementation of the Surface Response to Excitation (SuRE) method was conducted using a DSP circuit. Two different types of algorithms for training the neural network were used. Both have good results but the Scaled Conjugate Gradient presented highly improved results. If more data is collected, and the load applied in the beam is known, the neural network could be trained to detect intermediary points and load measurement on the beam. Sum of square of differences method only detected the presence of the load on the beam and couldn’t identify its location. 


\section{IMPLEMENTATION OF SURE METHOD FOR PART-BASED MACHINING PROCESS PERFORMANCE MONITORING}

6.1. Laser scanning vibrometer for remote process performance monitoring of automated manufacturing operations

\subsubsection{Introduction}

In this study the Surface Response to Excitation method (SuRE) is employed for remote performance monitoring of manufacturing operations. Feasibility of the SuRE for monitoring basic machining operations including cutting and drilling is studied. The proposed method has an advantage over conventional vision-based process performance monitoring systems. By choosing the scan points away from position of operation, it is possible to monitor the procedure. The reliability of this technique is examined through observing similar results after repeating the experimentation in similar conditions.

\subsubsection{Experimental setup}

A schematic of experimentation procedure is shown in the Figure 6-1.

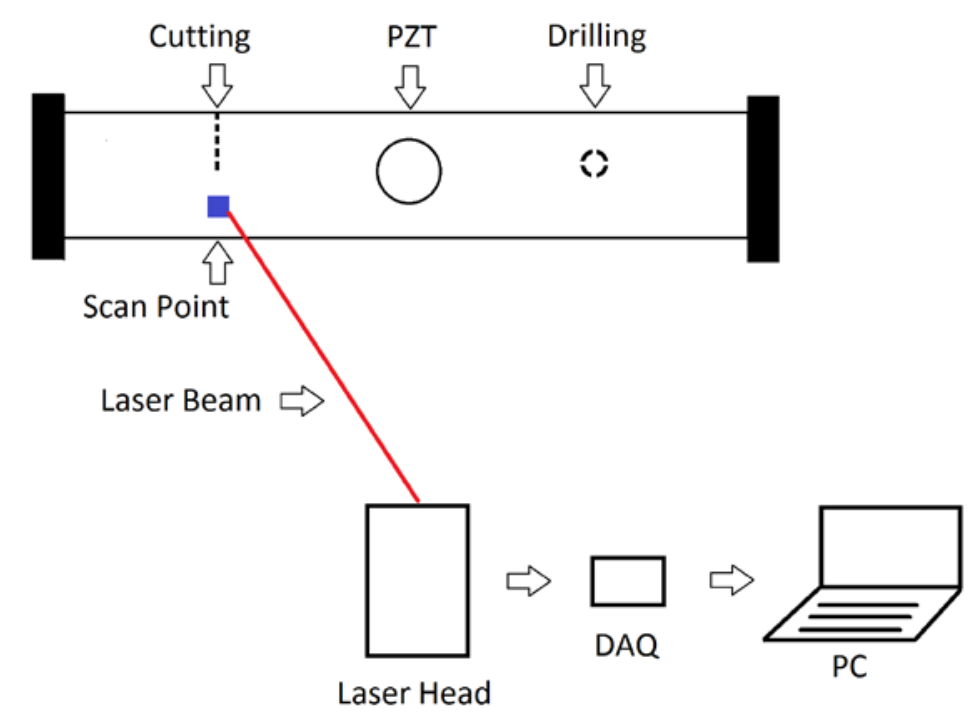

Figure 6-1 schematic of experimentation process 
The specimen that was used in this experimentation was a 2 in $\times 36 i n \times 1 / 16 i n$ aluminum beam. An APC piezoelectric element model D-.750"-2MHz-850 WFB was attached to the middle of the beam using the LOCTITE Hysol Product E-30CL epoxy adhesive. The aluminum beam was clamped in both ends within a wood frame. This wood frame and beam were fixed to the experiment table to avoid any movements during the operations. This way the scan points remained the same at all steps of the operation.

As it is shown in Figure 6-2, the scan grid includes 42 scan points that are arranged in 3 rows and 14 columns with the piezo element at the middle. The scan points were evenly distributed on both sides of the piezo and the scan points on the piezo were disabled. Size of scan area is $1.5 \mathrm{in} \times 21$ in. Scan grid includes two local operation regions where the manufacturing operations were conducted. Region 1 (R1) was used for cutting and region 2 (R2) was used for drilling. Scan points were marked on the work piece in order to make sure that at every step scans were exactly captured from the same points.

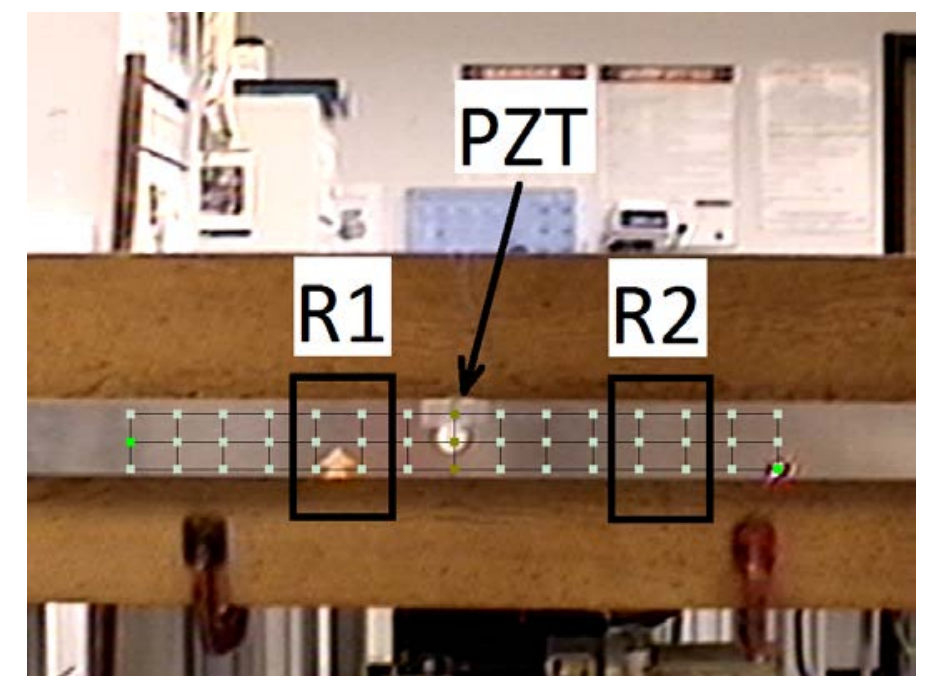

Figure 6-2 3×14 scan grid with 42 scan points; Region 1 for cutting (R1) and Region 2 (R2) for drilling 
At the first step of experimentation, cutting operation was performed at region 1 which is 3in left of the beam center. The depths of cut were $0.125 \mathrm{in}, 0.250$ in and 0.375 in respectively. The second operation was drilling at region 2, which was performed at 6 steps. Table 6-1 shows the diameter of drill cutter that was used to create the hole on the aluminum beam at every step:

Table 6-1: Drill hole diameters (inches) at during a 6 step drilling process

\begin{tabular}{cccccccc}
\hline 1 & 2 & 3 & 4 & 1 & 6 & 7 & 8 \\
\hline .09375 & .1093 & .125 & .1562 & .1875 & .21875 & 0.1875 & 0.250 \\
\hline
\end{tabular}

The PSV software of laser is capable of calculating the Fast Fourier Transform (FFT) of measured time data. The entire scan was captured in a single data file and exported for the post processing purpose. MATLAB was used to program and implement the SuRE algorithm. The NSSD values were calculated for all scan points for each step of operation. Contour map graphs of NSSD values were plotted to visualize the behavior of NSSDs and relate them to the manufacturing operation being studied.

\subsubsection{Results}

Figure 6-3 (b) shows the contour map of normalized squared differences for the cutting process. The red region of the contour map graph clearly identifies the cutting process on the upper edge of aluminum beam in the correct location. 

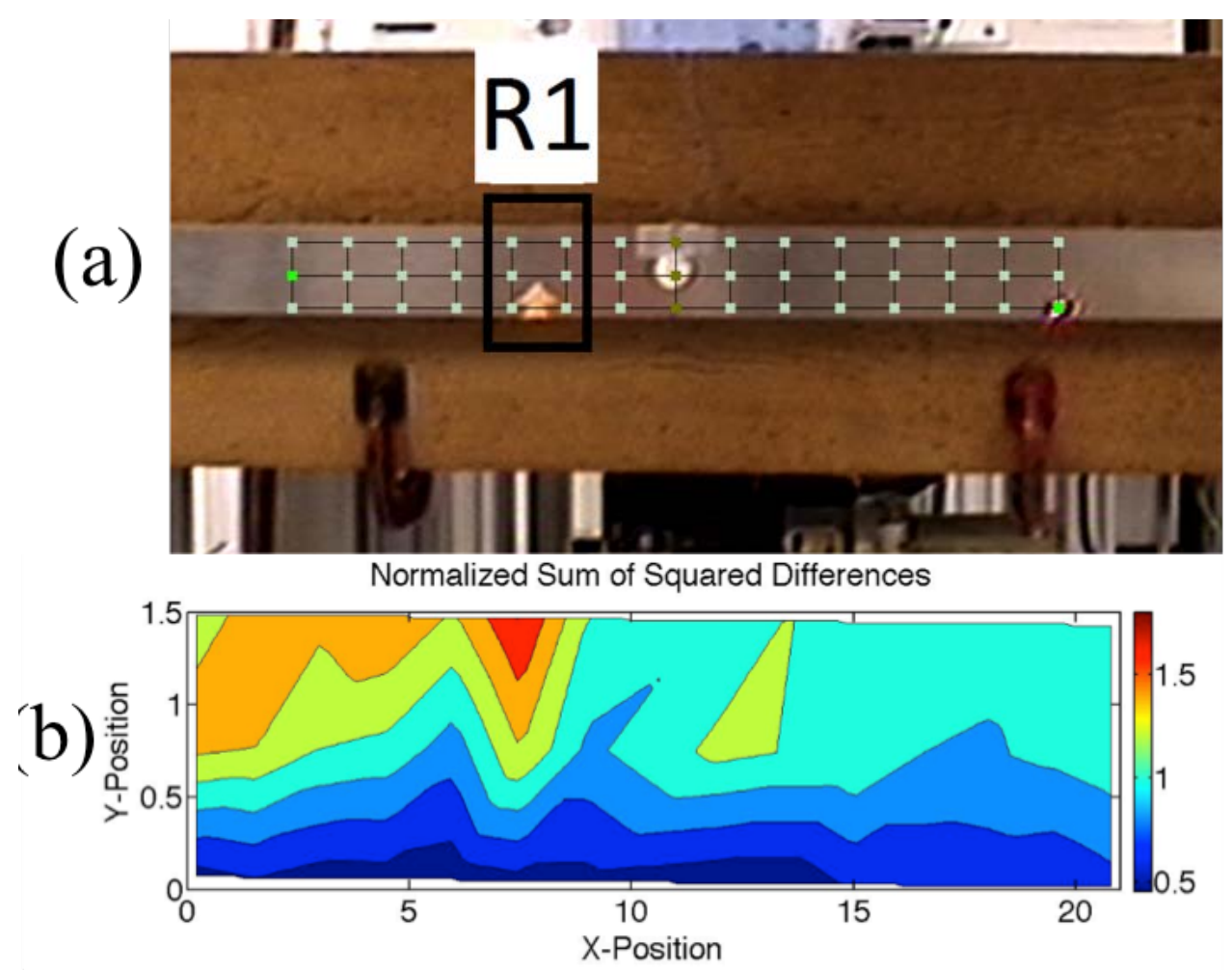

Figure 6-3 (a) The cutting process on the aluminum beam and (b) the corresponding contour map of normalized sum of squared differences

The interesting observation about the obtained results is that although the maximum changes in NSSD values are around the cutting edge, other areas of the beam also demonstrate changes in NSSD values to some extent. There is a yellow and orange area on the left side of the hot spot in Figure 6-3 (b) while the right of the cutting edge is mostly blue. This means the NSSD values have undergone more changes in the left hadside of the cutting edge than the right-hand-side. It makes sense knowing that the piezoelectric is located at the center of the beam and the cutting region R1 that is located on the left side of the piezo actuator. The piezo element excited the surface waves and they traveled from center to left side of the beam. Once the cutting process was performed, the presence of the cutting edge on the path of surface waves caused change 
in their propagation characteristics to the rest of the beam. This fact was reflected in the NSSD values increasing to some extent in the orange and yellow area of the in Figure 6-4. Another observation during the experiments was that NSSDs values of every scan points were increased after every step of the cutting process. This phenomenon is shown at the for a scan point in the red area close to the cutting operation.

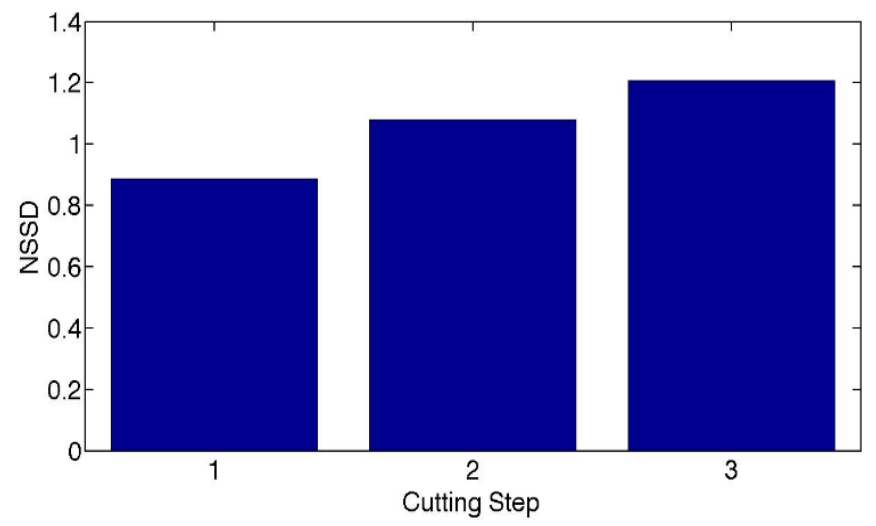

Figure 6-4 Normalized sum of squared differences (NSSDs) of scan point $[2,6]$ at during the cutting operation

The NSSD values showed similar pattern even in the scan points that were not adjacent to the cutting edge which could be used to monitor the performance of the cutting operation in automated manufacturing systems.

The Figure 6-5 shows an example of the NSSDs on the left of the cut edge within the yellow-orange area of Figure 6-3 (b). 


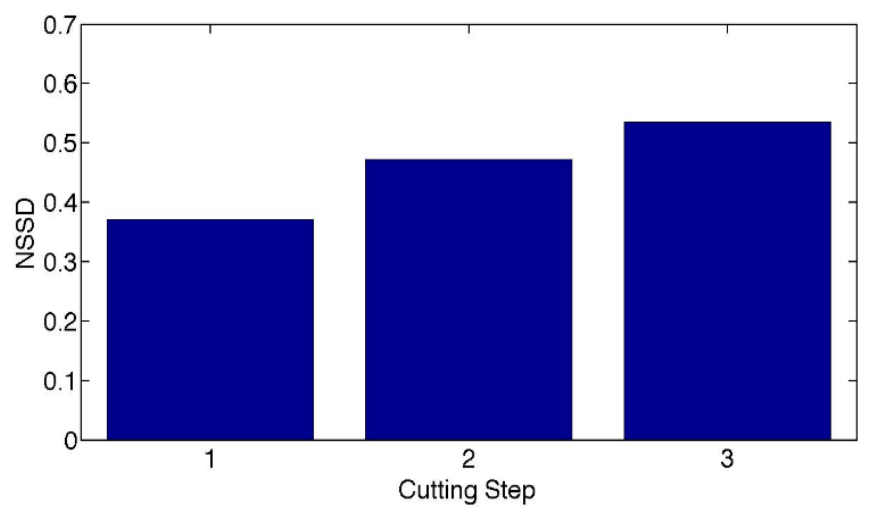

Figure 6-5 Normalized sum of squared differences (NSSDs) of scan point [1,3] at during the cutting operation

Due to the observation of Figure 6-5, the NSSD values could be collected away from where the process is happening. The importance of this point is that in industrial manufacturing the area close to the cutting process usually is covered with chips and cooling agent and could not be used for the data acquisition. Even in the vision-based systems cleaning and removing the work-piece is the biggest challenge for commercial application.

The drilling process was performed through 8 steps on the region R2 on the beam. Figure 6-6 shows how the NSSD values. The red area shows that the maximum values of NSSDs are occurred at adjacent to the drill hole. There is also a yellow area on the right side of the red spot. The presence of this area shows that the wave propagation characteristics are changed after waves travel through the drill hole area. 

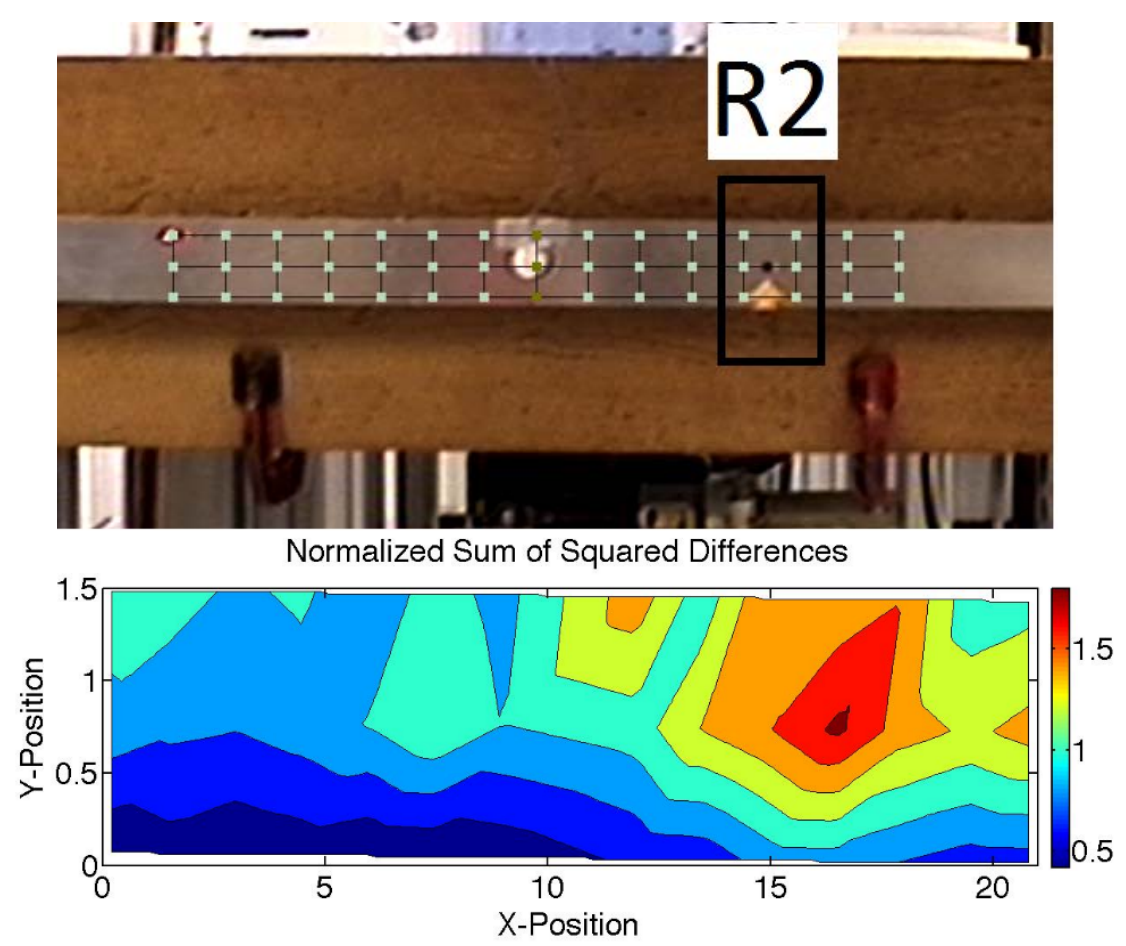

Figure 6-6 The drilling process on the aluminum beam and the corresponding contour map of normalized sum of squared differences

In the Figure 6-7 NSSD values at each step of drilling process on a scan point within the red area of Figure 6-6 is shown. A similar increasing behavior could be observed compared to the cutting process.

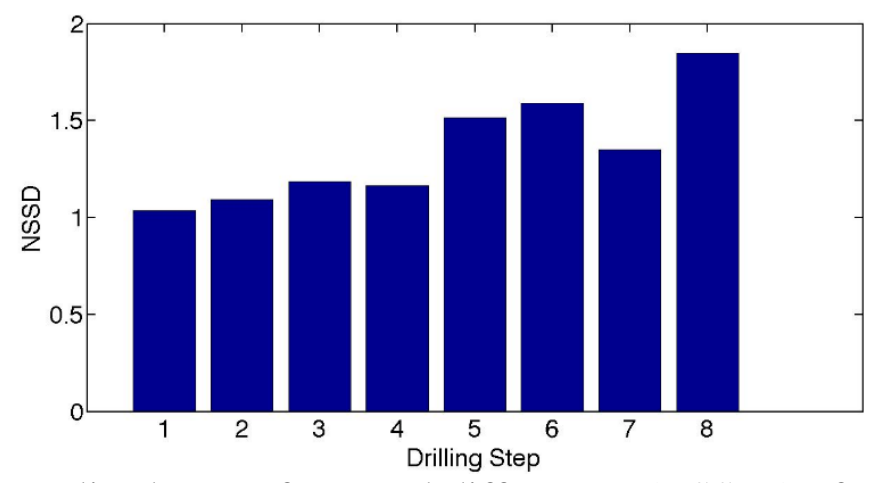

Figure 6-7 Normalized sum of squared differences (NSSDs) of scan point [2.11] at during the drilling operation

The behavior is almost consistent throughout the entire experimentation (other than step 7) and NSSD values almost doubled at the last step. Therefore, they could be 
correlated to the hole-diameter and be employed in development of drilling process performance monitoring.

The inconsistency that was observed in the step 7 of the drilling process was due to the noise in laser vibrometer measurements. During this study, the scans were performed completely automatically. After each step of operations, the LSV automatically scanned the entire grid and it switched from one scan point to another without any interference. Although this is much more convenient to perform the scans, it leaves the vulnerability to the presence of noise in the measurements and could lead to reduced reliability of the method.

\subsubsection{Conclusion}

An automated process monitoring of two basic machining processes was developed through modifying of an already existing structural health monitoring technique. Once an efficient SHM process is developed, that could be implemented with minimal expense and equipment, it would be possible to inspect several manufacturing processes. The advantages of such a method are no- interference with the manufacturing process and evaluating tool or product condition with complicated configuration. Due to simplicity of the proposed algorithm, the method avoided heavy computational costs and expensive equipment required in methods like machine vision-based SHM. Due to the non-contact nature of the laser beam, it was possible to collect data from any point on the work-piece and locate the best possible points to locate the piezoelectric elements. The method's implementation in real world automated manufacturing processes requires considerable more experimentation. 
6.2. An Experimental Investigation of Part-based Process Performance Monitoring Method for Machining Process Monitoring

\subsubsection{Introduction}

In this study, part-based process performance monitoring (PbPPM) is studied for application in remote performance monitoring of machining operations. Earlier in chapter 4 the numerical simulations using COMSOL revealed the pattern of interaction of surface wave with each machining operation. The results of this study showed that sums of square of differences (SSDs) values were sensitive to machining operation and increased at each step of operation. The purpose of current study is to validate those results experimentally. The laser scanning vibrometer (LSV) collected data from a grid of scan points overlapping with the probe points of numerical study. Results revealed that the color map diagrams of the SSDs successfully indicated the presence of operation on the work piece, its location, and the extent and type of operation. The reliability of this technique is examined by observing similar results after repeating the experimentation in comparable conditions.

\subsubsection{Experimental setup}

The specimen used was a (2in) $\times(36 i n)$ aluminum beam with (1/16in) thickness. Figure 6-8 shows a schematic of experimentation set up. 


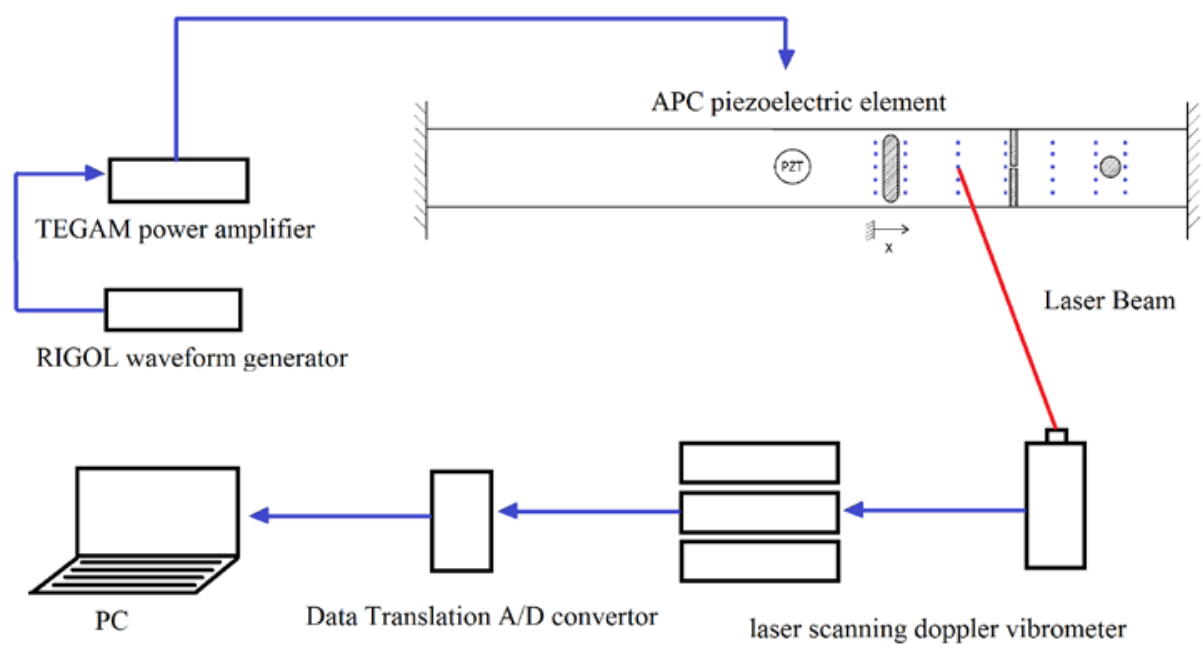

Figure 6-8 Schematic of experimentation set up

The choice of non-contact sensing system allows the analysis of behavior of SSD values over the entire beam surface. Figure 6-9 shows the scan grid, which includes 35 scan points that are arranged in 5 rows and 7 columns. Dimension of scan area is (2in) $\times(7.5 \mathrm{in})$. Scan grid is designed to accommodate three local operation areas shown in the figure. First the drilling was performed in R1 section, then cutting was done in R2 area and finally milling operation was conducted in R3 area. In order to make sure to scan exactly the same points after each operation, the scan points were marked on the work piece.

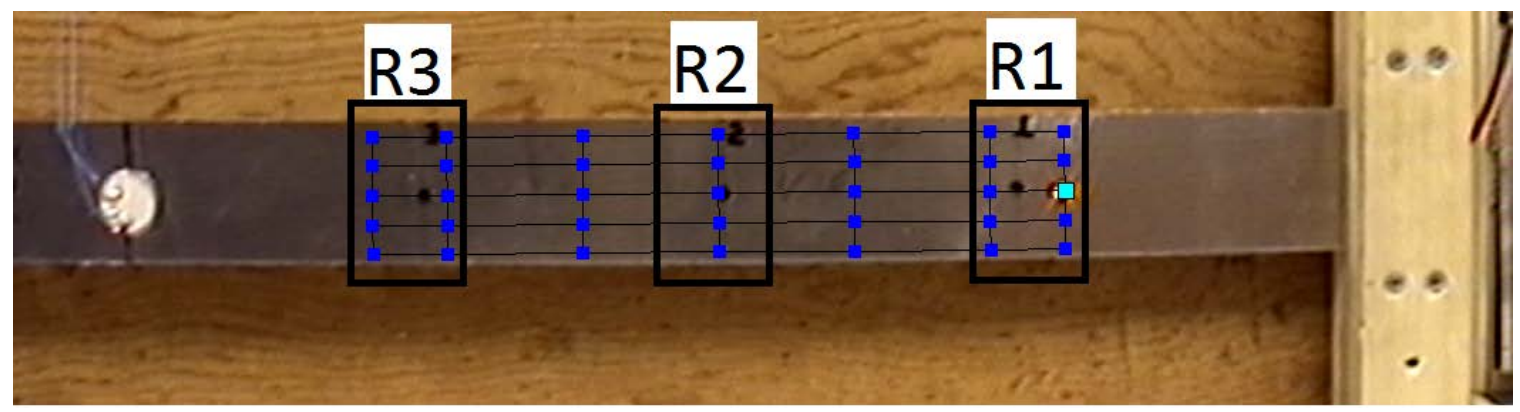

Figure 6-9 scan grid including 35 scan points on a $5 \times 7$ grid with region 1 for drilling (R1), region 2 for cutting (R2), and region 3 for milling (R3) 
Due to the limitation of sampling frequency of $\mathrm{A} / \mathrm{D}$ converter of laser junction box, an external data acquisition system is used. Data Translation simultaneous A/D convertor model DT9832-A is employed to capture the peak holds of the transfer function for the frequency input sweep sine wave. The maximum sampling rate of the device was set to 1000000 samples per second, which lets a maximum frequency of 400 $\mathrm{KHz}$ to be sampled. Since the SuRE algorithm requires the frequency domain data, the FFT of the input data is used. The DT9832-A has a built in FFT package. The FFT size was set to 16384 and Hanning window function was used as smoothing window.

\subsubsection{Results}

Both the drilling and cutting processes were performed in 8 steps. Table 6-2 shows the cutter diameter in drilling operation and depth of cut in cutting operation at each step of the corresponding process:

Table 6-2 Cutter diameter in drilling process and Depth of cut in cutting process

\begin{tabular}{lllllllll}
\hline Step 1 & 2 & 3 & 4 & 5 & 6 & 7 & 8 & \\
& & & & & & & & \\
\hline Diameter (in) & $1 / 16$ & $7 / 64$ & $9 / 64$ & $5 / 32$ & $3 / 16$ & $15 / 64$ & $1 / 4$ & $5 / 16$ \\
& & & & & & & & \\
\hline Depth (in) & 0.125 & 0.25 & 0.375 & 0.5 & 0.625 & 0.75 & 0.875 & 1 \\
& & & & & & & & \\
\hline
\end{tabular}

Figure 6-10 (a) shows the color map plots of the sum of squared differences. The red area identifies the location of highest SSD values. Figure 6-10 (b) shows the threedimensional surface representing the change in the SSD values. The peak is clearly distinguished in the area 1 at the exact location of drilling operation. 

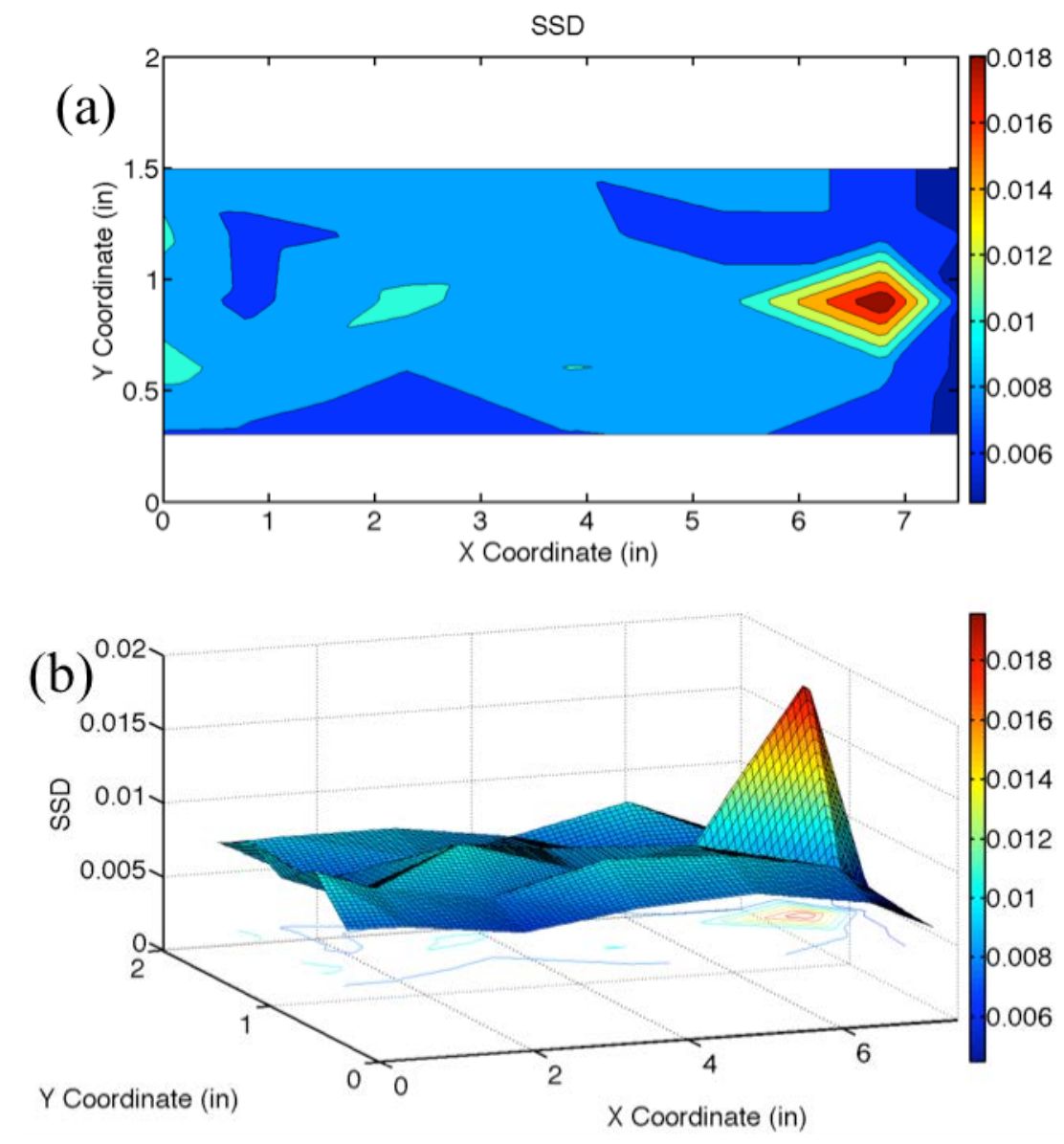

Figure 6-10 (a) Color map diagram of sum of squared of differences for drill process; (b) three-dimensional graph of SSD values for drilling process

The maximum increase in SSDs was in the location of operation. The behavior was consistent and nonlinear. Figure 6-11 shows the SSD values and the uncertainty in measurement in the form of error bar diagrams for the drilling and cutting operation. 

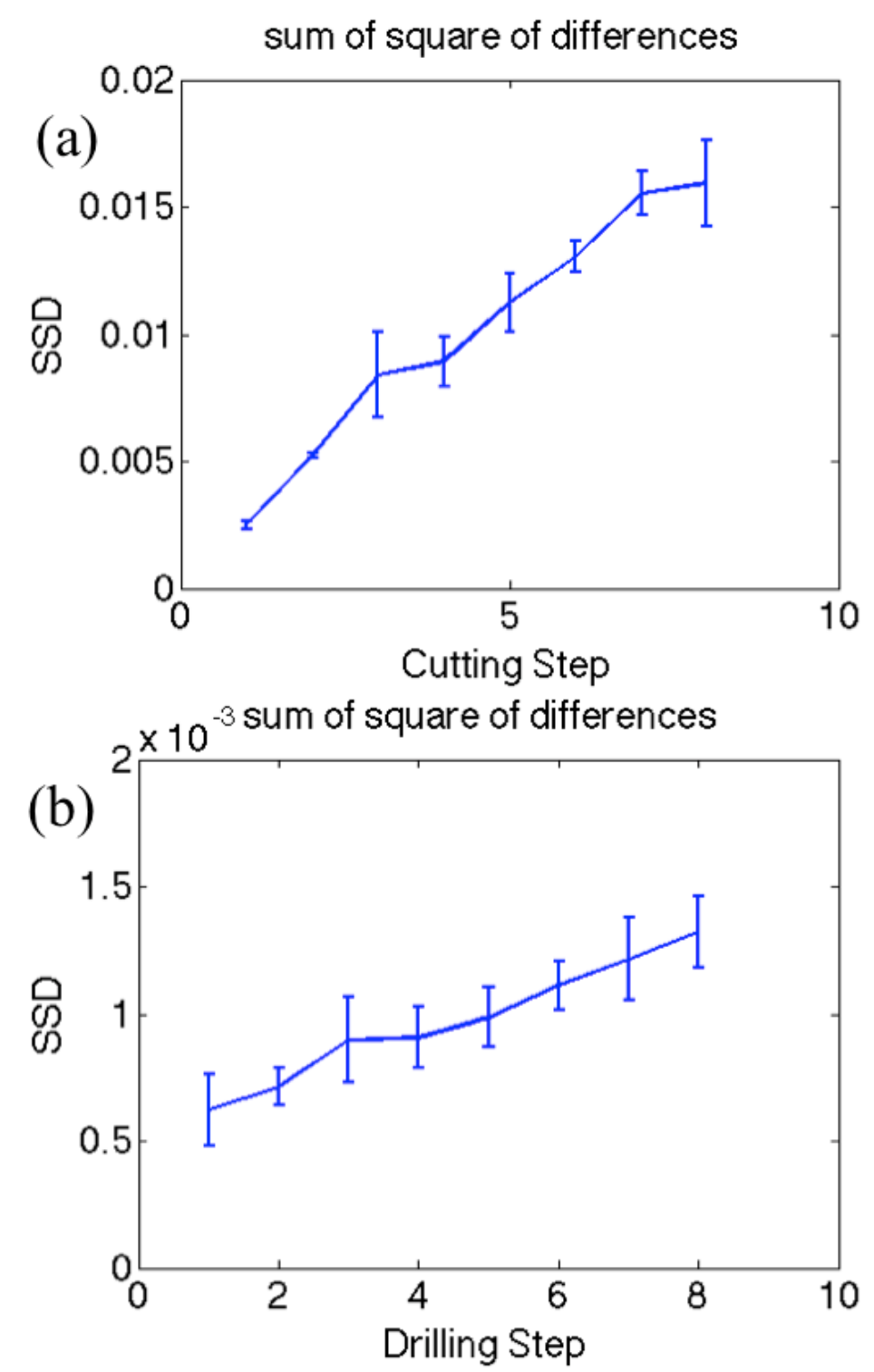
process

Figure 6-11 values and uncertainty during 8 steps of (a) drilling (b) and cutting

In order to present a more general trend of behavior of SSD values, the SSD values of entire $6^{\text {th }}$ column and $4^{\text {th }}$ row of measurement points are shown in the Figure 6-12. Since laser vibrometer has a low signal to noise ratio, in some cases measurements were influenced by noise and this problem cause abrupt increase in SSD values. 

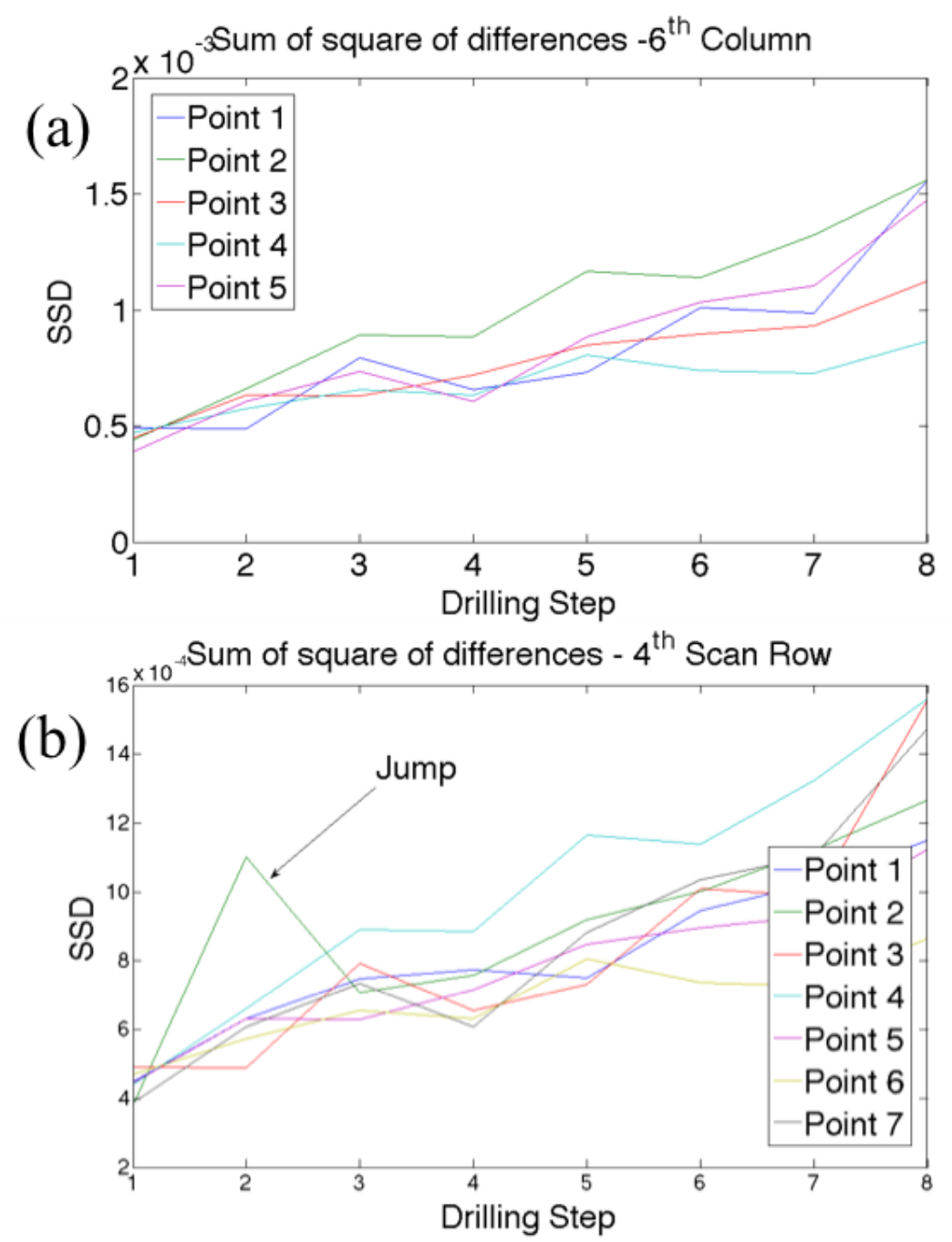

Figure 6-12 Sum of square of differences for measurement points in column 6 (top) and row 4 (bottom)

Figure 6-13 (a) shows the contour plots of sum of squared differences for cutting operation. The cutting operation was a symmetric double cut type from both ends of the beam's width. The red spot reveals the local increase of SSD values in both sides of cutting area. Also as the depth of cut increased during each step of operation, the extent of red spot increased as well. Figure 6-13 (b) shows the increased SSD values in the form of a three-dimensional surface plot. 

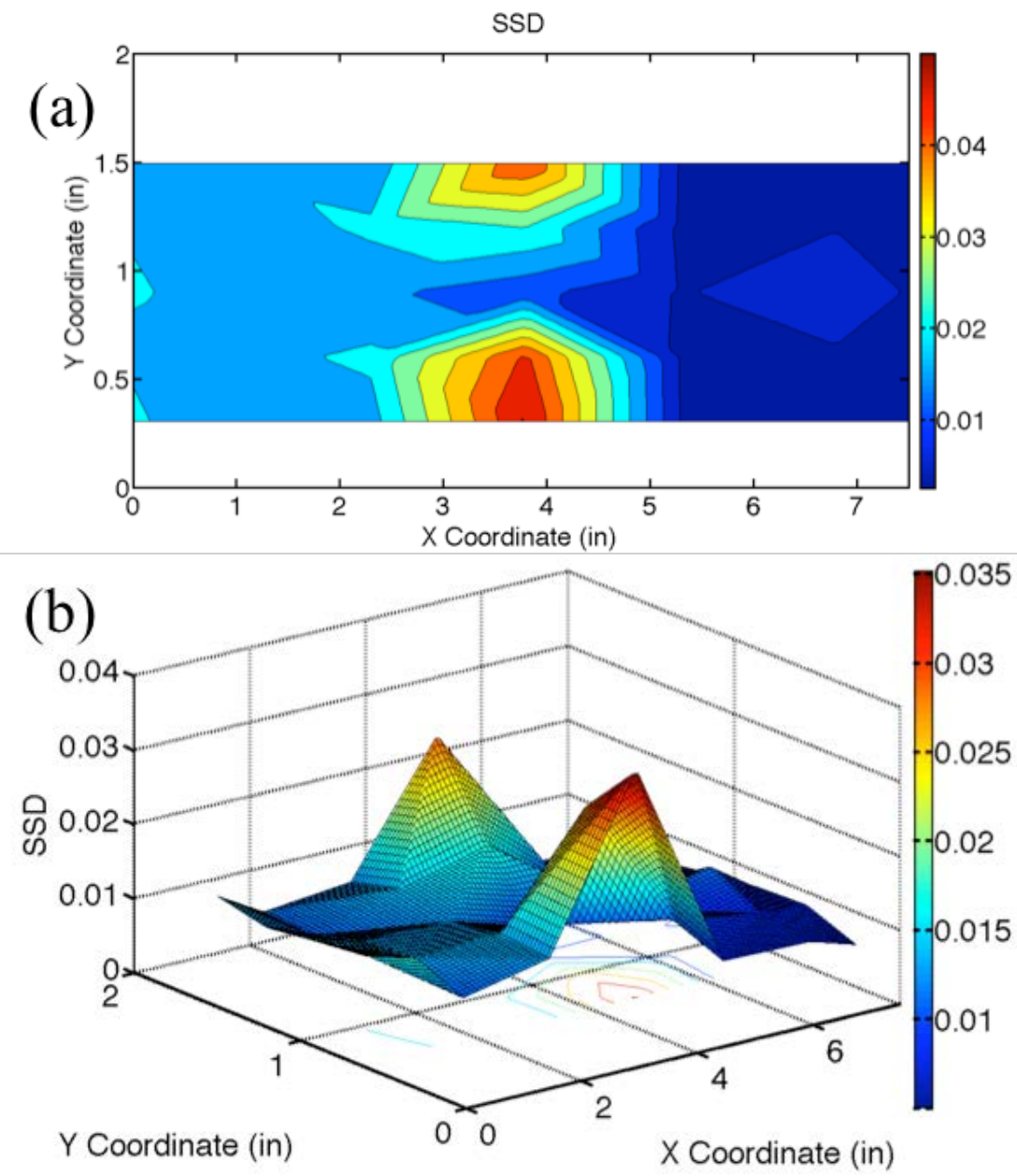

Figure 6-13 contour plots of SSD for (a) step 2 with 0.25 depht and (b) step 6 with 0.75 depth

\subsubsection{Conclusion}

Our study proved that the location and dimensions of completed multiple machining operation may be inspected by using the SuRE method. The sensitivity of the PbPPM method was very good in drilling and cutting operations. The sensitivity of the method decreased when the milling operations were evaluated. The numerical simulations of chapter 4 identified the optimized parameters of frequency sweep. Study of SSDs also showed that their behavior is consistent through the entire surface of work piece. The SSD values showed the sensitivity to three basic parameters of the slot; 
namely length, depth and width. The sensitivity was very good when the length and width changed. Sensitivity was less when the depth changed.

6.3. A non-contact method for part-based process performance monitoring in end milling operations

\subsubsection{Introduction}

PbPPM method was employed for inspecting the completed milling operations. Laser-scanning vibrometer was used to monitor the propagation of high-frequency surface waves on an aluminum plate in presence of milling operation. Due to the noncontact nature of this study no interference occurs with the milling process. Results showed that the PbPPM method could evaluate the completed metal removal.

\subsubsection{Experimentation setup}

An aluminum plate was used in this experimentation with $(4$ in)(11 in)(0.4 in) dimensions. Figure 6-14 shows the experimental setup for SuRE system. 


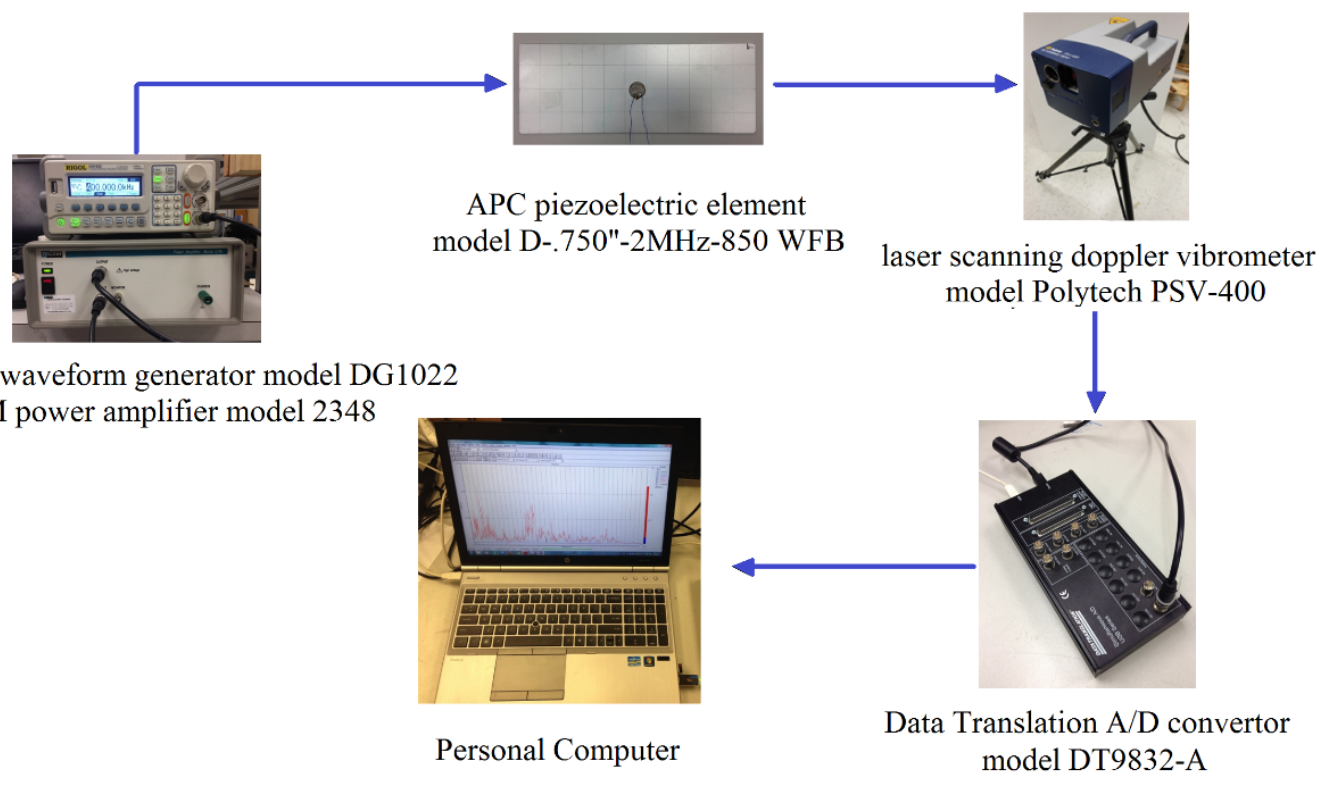

RIGOL waveform generator model DG1022 TEGAM power amplifier model 2348

Personal Computer

Figure 6-14 Experimentation setup for SuRE system

The scan grid included 50 scan points that were arranged in 5 rows and 10 columns with the piezo element in the middle. Dimensions of scan area were (3.5in) by (9.5in). Scan grid in the both sides of piezoelectric included five columns of scan points (Figure 6-15). In total 10 milling operation were conducted in the areas between the scan points. In each operation the region is located between two scan columns. The frequency rang was set to $20-400 \mathrm{kHz}$. 


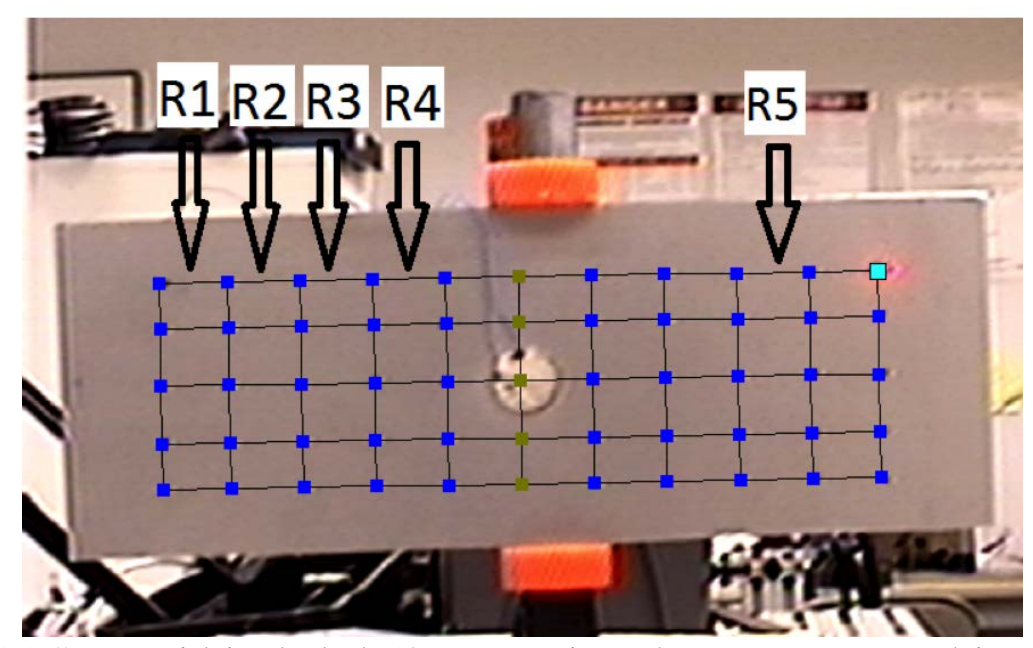

Figure 6-15 Scan grid included 50 scan points that were arranged in 5 rows and 10 columns with the piezo element in the middle

\subsubsection{Results}

The purpose of this study was to evaluate the potential capacity of PbPPM method for remote process monitoring of milling operation. Four different sizes of High Speed Steel (HSS) milling heads shown in Table 6-3 were used. Experiments were divided into three categories for analysis of effect of length, depth and width of the milling on measurements.

Table 6-3 HSS flat head milling heads being used for the experiments of this study

\begin{tabular}{lllll}
\hline Milling Head & First & Second & Third & Fourth \\
\hline Size (in) & $1 / 8 * 3 / 8$ & $3 / 16 * 3 / 8$ & $5 / 16 * 3 / 8$ & 3rd 3/8 *3/8 \\
\hline
\end{tabular}

For each study the milling operations were performed in a stepwise approach and at each step the specimen was scanned. In the case of milling length analysis, the milling operation was performed at three steps using an ACRA milling machine. The HSS milling head sizes in this case was $3 / 8 \times 3 / 8$ (in). The advantage of using laser-scanning 
vibrometer could be realized in real time analysis applications. While scan points close to the operation are covered with the metal chip and coolant, the scan points away from the operation are available for remote data acquisition (Figure 6-16).

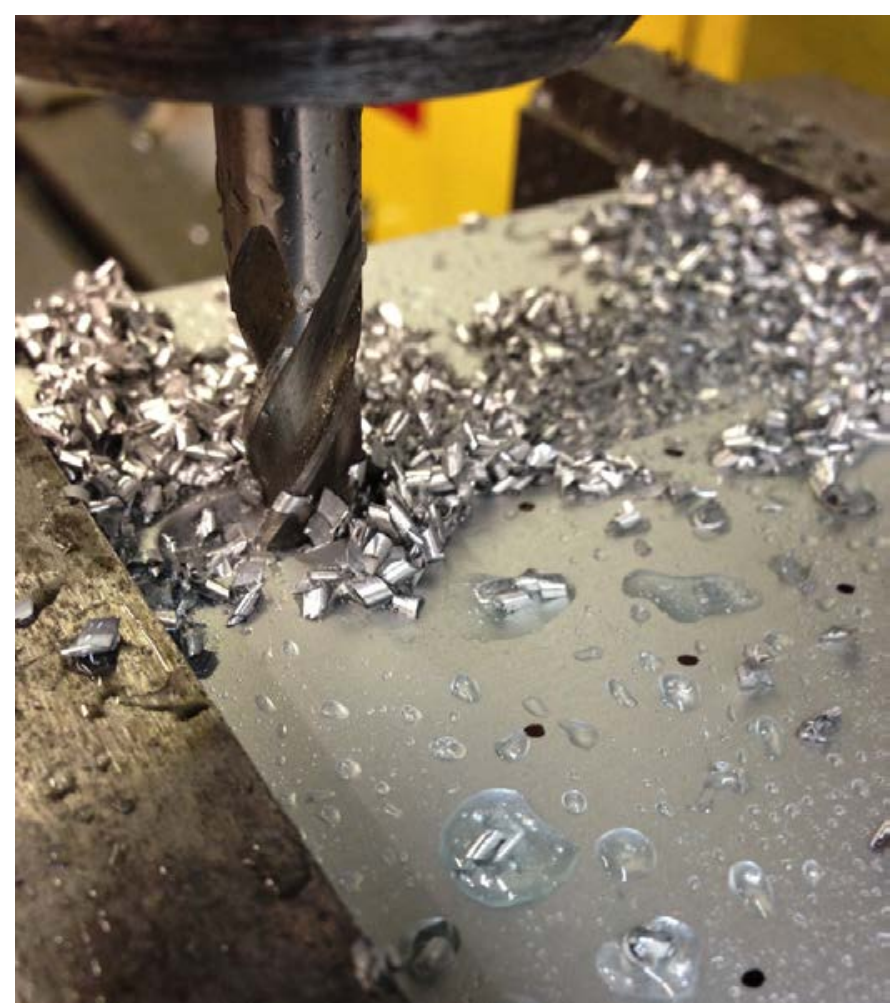

Figure 6-16 The coolant and cutting chips are covering the scan points in area close to milling operation

The data for every scan point was analyzed in the frequency domain. Before any milling operation the reference scan was performed on the intact plate. The reference scan was repeated in order to evaluate the consistency of the procedure. Then, the milling operation was performed for study the effect of length, depth and width of milling on the measurements. The milling for each case was performed in certain number of steps according to the Table 6-4. After every step of operation, a laser scan was captured from the same scan points on the plate. 
Table 6-4 HSS flat milling heads used for the experiments of this study

\begin{tabular}{lcccc}
\hline Milling step & First & Second & Third & Forth \\
\hline Length (in) & 0.375 & 1.475 & 4.51 & - \\
\hline Depth (in) & 0.03 & 0.06 & 0.09 & - \\
\hline Width (in) & $1 / 8$ & $3 / 16$ & $5 / 16$ & $3 / 8$ \\
\hline
\end{tabular}

Next figure compares the measured frequency spectrums from the first and second reference scan. Majority of peaks and valleys of both reference spectrum overlap in Figure 6-17 (a) while after the milling in Figure 6-17 (b) the captured spectrum don’t match to the reference.

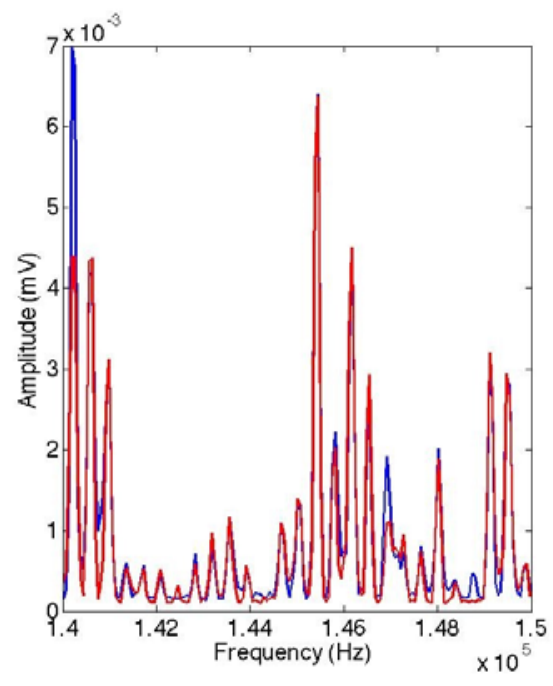

(a)

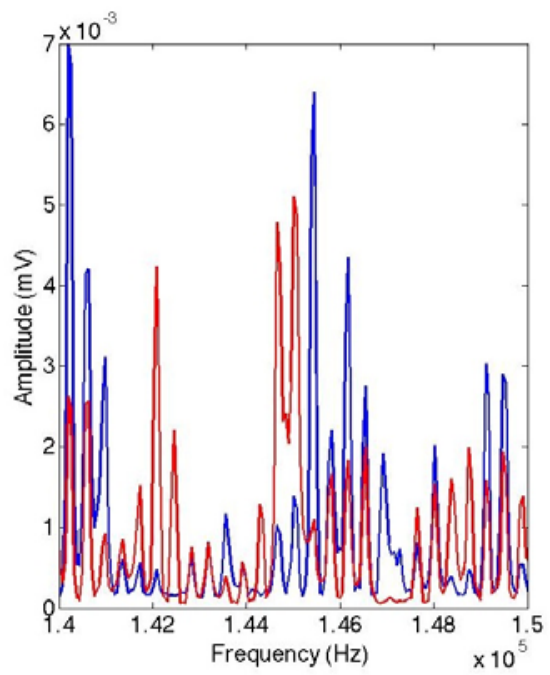

(b)

Figure 6-17 $1^{\text {st }}$ Reference spectrum vs. $2^{\text {nd }}$ reference spectrum, 5 (b) $1^{\text {st }}$ reference spectrum vs. $1^{\text {st }}$ milling spectrum

According to the part-based process performance monitoring (SuRE) the sum of squared differences (SSDs) of each spectrum from reference spectrum was calculated for 
all scan point at each step of milling operation. Since the SSD values were calculated for all scan points, contour map plot of those values at every step could give a visual understanding of the procedure. The change in the frequency spectrum could be observed in three different ways. Peaks dropped, raised and drifted with respect to the original position in the reference scan.

\subsubsection{1. $\quad$ Study of milling length}

The study of the length of milling was conducted on the left side of the plate within areas R1 to R4 (Figure 6-15) for each of the four milling sizes. Contour map of the SSD values over the surface of the plate is shown in Figure 6-18. Considering that the area R1 was located on the left side of the plate and the operation was performed on this area, the red spot of the contour map successfully identifies of the operation on the plate.

(a)

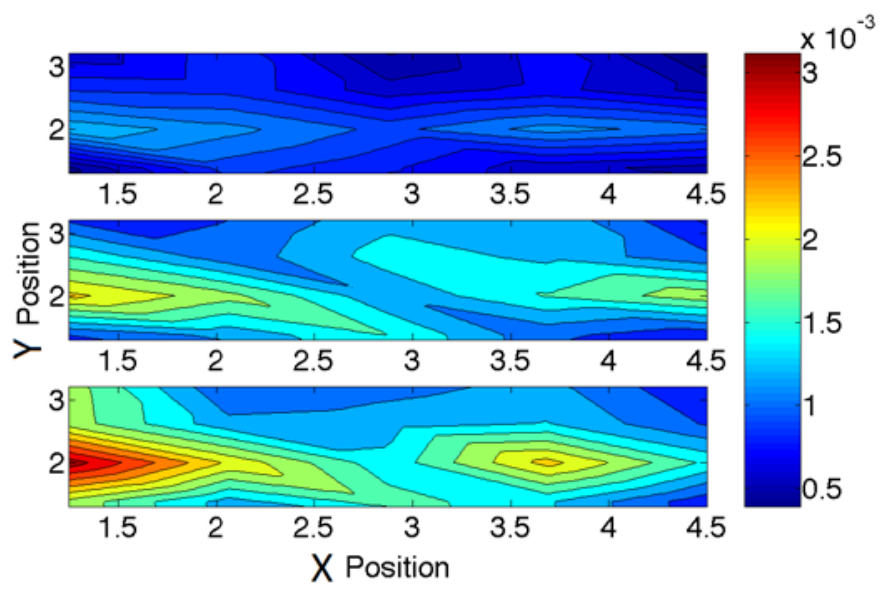

Figure 6-18 Contour map of SSD values on the scan area for the (a) 1st milling (b) $2^{\text {nd }}$ milling (c) $3^{\text {rd }}$ milling 


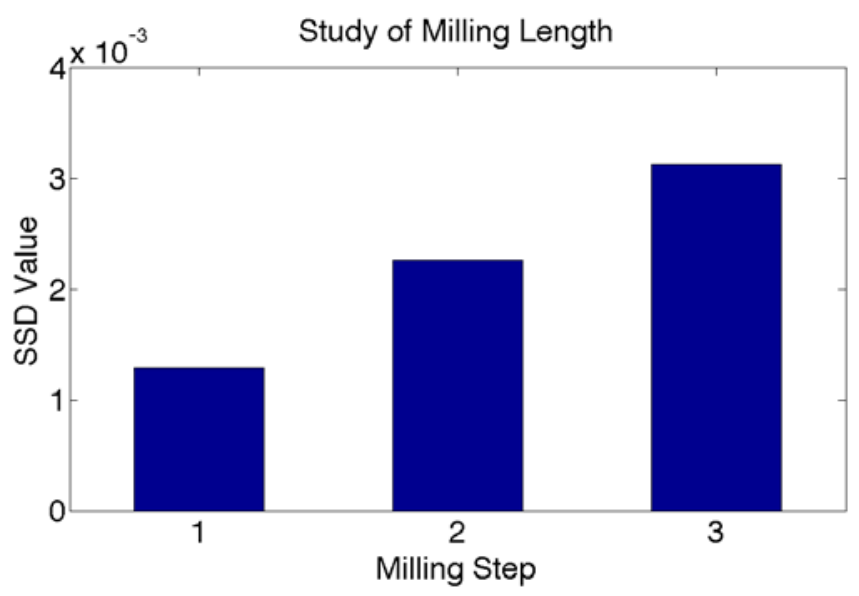

Figure 6-19 Maximum SSD values on the scan area for the study of milling length (a) 1st milling step (b) 2nd milling step (c) 3rd milling step

At every step, the maximum values of SSDs occurred close to the location of operation. A secondary weaker red spot is also appeared away from the first one. The study revealed that the SSD values in this area show similar pattern to those close to the location of operation. These values potentially could be used for monitoring the operation. Figure 6-19 demonstrates the SSD values increase by increasing the length of milling.

\subsubsection{2. $\quad$ Study of milling depth}

The operation for study of the depth was performed in the area R5 (Figure 6-15), which is located on the right side of the plate within $3^{\text {rd }}$ and $4^{\text {th }}$ columns of the scan grid. In Figure 6-20 the three-dimensional contour map of the SSD values correctly identifies the location of the milling operation on the plate.

This is a property of SuRE method that is useful in damage localization in SHM applications. It could be used to monitor the correct location of each operation in automated machining monitoring applications. Dimensional accuracy is another 
important property in machining. Figure 6-21 includes SSD values for the three consequent steps of the millings where the depth of milling has increased in each step according Table 6-4. As the milling depth increases, the maximum value of the SSDs also increases.

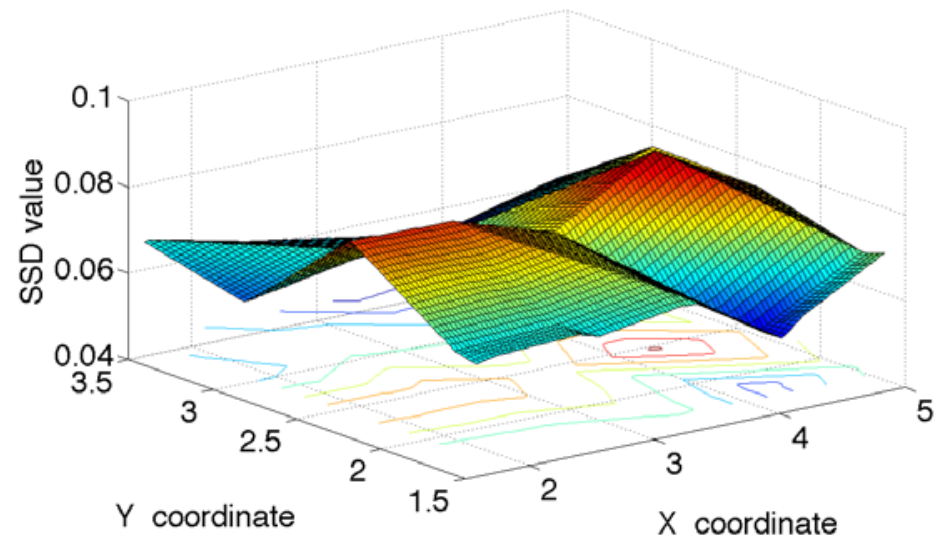

Figure 6-20 Three-dimensional demonstration of SSD values on the scan area

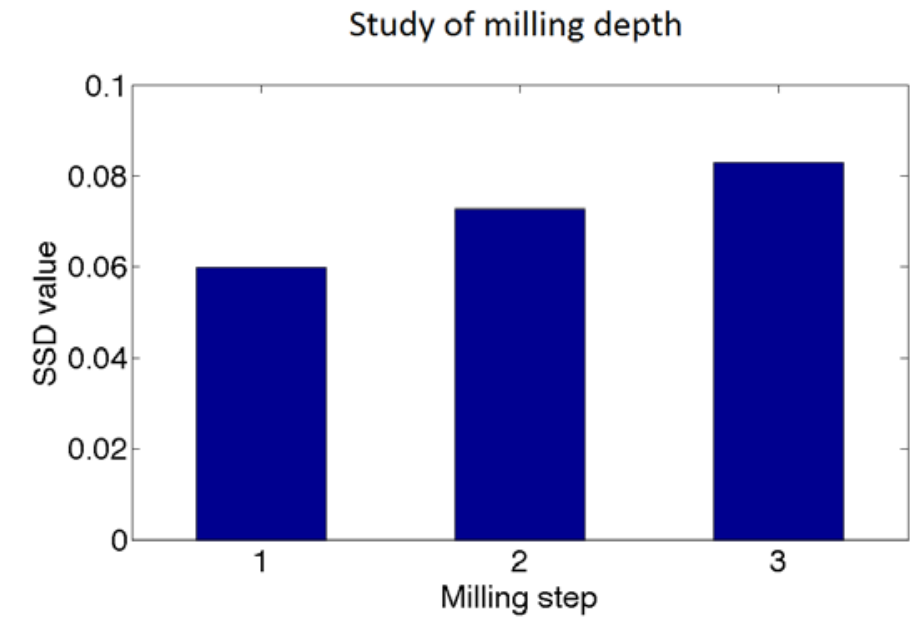

Figure 6-21 SSD values versus depth of milling increasing in 3 steps

\subsubsection{Study of milling width}

The study of milling width was performed in 4 steps in the R5 region between $3^{\text {rd }}$ and $4^{\text {th }}$ scan columns (Figure 6-15). Although the change in the value of SSD in the study 
of milling depth had similar value to those of depth and length, due to the larger SSD values in this level of study, the relative value of changes in the SSD seems to be small in Figure 6-22.

Therefore, among all three basic parameters that were studied, SSD values showed the least sensitivity to the milling depth. Especially, in scan points close to edges of the plate the behavior of SSDs was not consistent. But if the position of the scan point is chosen carefully in central areas of the plate, a more reasonable behavior could be observed. Therefore, if for any reason one of the operations was not performed, it could be detected before specimen moves on to the next operation.

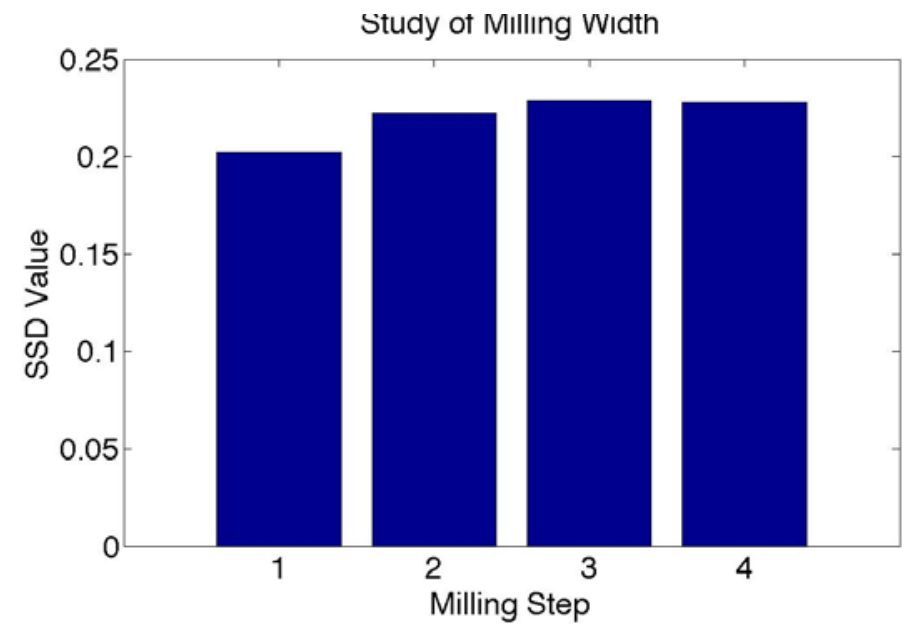

Figure 6-22 7.10 SSD values versus depth of milling increasing in 4 steps

\subsubsection{Conclusion}

The purpose of this study was to monitor the quality of the completed milling operations using PbPPM technique. The variation of the spectrums was evaluated by calculating the sum of the squares of differences (SSD) between the reference and spectrums after each machining operation. The SSD values quickly increased when the size of the slot changed. The SSD values showed the sensitivity to three basic parameters 
of the slot; namely length, depth and width. The sensitivity was very good against length and width. It was minimum to depth change.

Instead of monitoring the machining operation with sensors, the performance of the completed operation may be evaluated with piezoelectric sensors attached to the workpiece in milling operations. This would reduce the cost of inspection and bring the initial investment to a minimum. In addition, the same sensors may stay on the workpiece for monitoring application in the rest of the manufacturing process.

6.4. Surface Response to Excitation Method for Part-Based Manufacturing Process Monitoring of 3D Objects

\subsubsection{Introduction}

The purpose of this study was to investigate the efficiency of PbPPM method through various implementation techniques in multi-dimensional machining monitoring applications. Various possible scenarios of orientation of where the excitation, monitoring and operation are not located the same plane, were considered and the reliability of technique was proved in each case. 


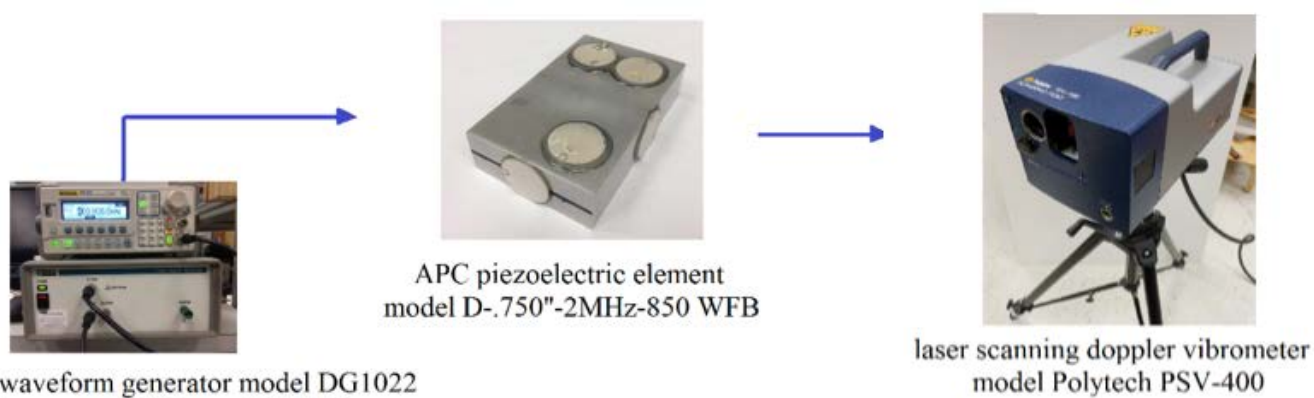

RIGOL waveform generator model DG1022

TEGAM power amplifier model 2348

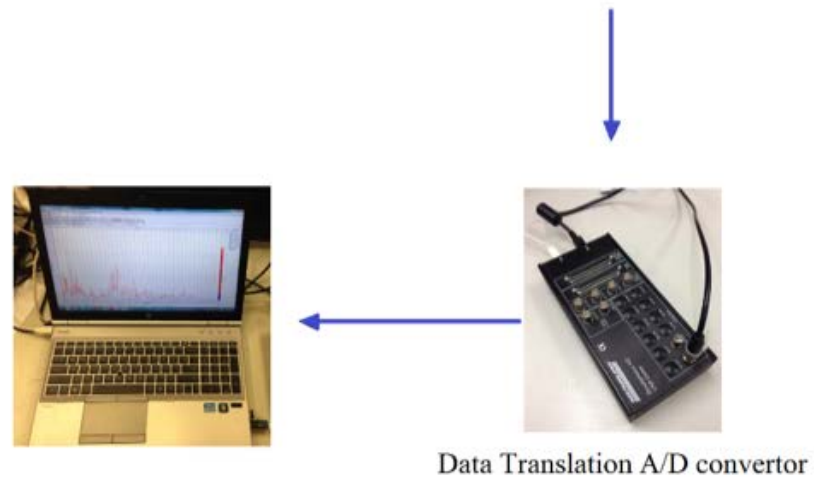

Figure 6-23 Laser sensor-based implementation procedure of part-based process performance monitoring

\subsubsection{Experimental setup}

The performance of the PbPPM method was evaluated using threeimplementation technique. The schematic of Figure 6-23 shows the laser-sensor-based implementation procedure using laboratory equipment.

\subsubsection{Piezoelectric-Based Implementation}

Five PZT elements were bonded on the $3.2 \times 2 \times 0.75$ (in) aluminum block (Figure 6-24). Three piezos were bonded to the top surface were the operation was introduced. Also one piezo at the center of each of the two side perpendicular planes (bottom and left) bonded. The surface waves are excited in a stepwise manner over a certain 
frequency range between $20-400 \mathrm{kHz}$ the type of excitation is a sweep sine wave over. After the reference data measurement, the damage to the block was introduced as a surface milling $1 \times 3 / 16 \times .05$ (in). The sensing performed via both contact and non-contact approaches. In the contact method, after choosing the exciter PZT, the remaining piezoelectric electric elements were used as sensors. The reference data was captured for each of the five piezos used as exciter one by one.
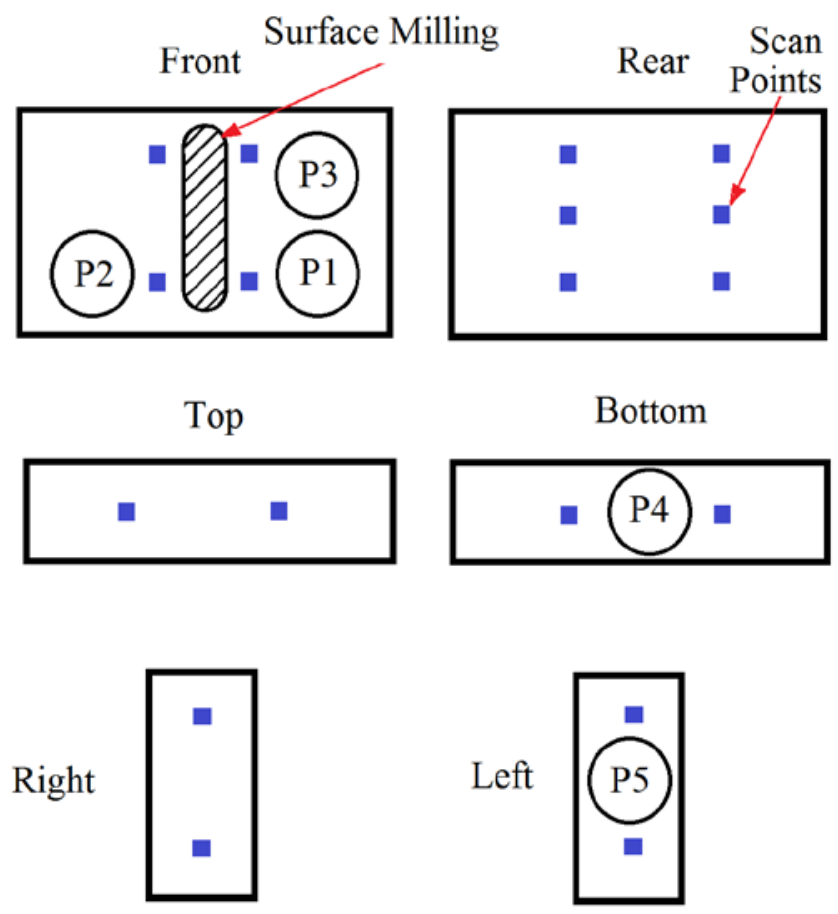

Figure 6-24 Schematic of aluminum block with five piezoelectric elements bonded on three perpendicular faces and laser scan points

\subsubsection{Laser Vibrometer-Based Implementation}

In the case of non-contact sensing, the scanning head of laser scanning Doppler vibrometer (LSDV) model Polytech PSV-400 remotely measured the surface vibrations. A grid of scan points, (shown in Figure 6-24) were specified for each of the six facets of the block and data was measured from these points. Data Translation simultaneous A/D 
convertor model DT9832-A was employed as the data acquisition module. In the case of multiple sensing points, the simultaneous analog input operation of the high performance DT9832-A module allowed reducing the acquisition time. In this study Spectra PLUS-DT software designed for the data translation line of USB modules was used. The real-time Fast Fourier Transform (FFT) analysis property of software combined with the peak hold option was used to capture the signature frequency transfer function. Data was exported for post processing in MATLAB using the surface response to excitation algorithm.

\subsubsection{3. $\quad$ Embedded Device-Based Implementation}

To reduce the time and cost of implementation for practical applications an embedded device based on PIC’s 16-bit Digital Signal Controller was used. For more information on the details of implementation techniques please refer to 3.6.1.

\subsubsection{Results}

The results are presented in the three upcoming subsections for each of the implementation techniques described in the previous chapters.

\subsubsection{Contact Sensing Piezoelectric-based implementation}

Using PbPPM method the reference data was measured first. Figure 6-25 shows the baseline spectral data when piezo 1 was exciting measured from piezos 2-5. Every measurement of this study was repeated three times to verify the consistency of spectrums and identify any possible uncertainty in measurements. The basic concept of 
PbPPM method is shown in Figure 6-25. Where all of the spectrums overlap while no change has occurred in the condition of structure. Another observation in Figure 6-25 is that the majority of dominant peaks are in the range of less than $200 \mathrm{kHz}$.

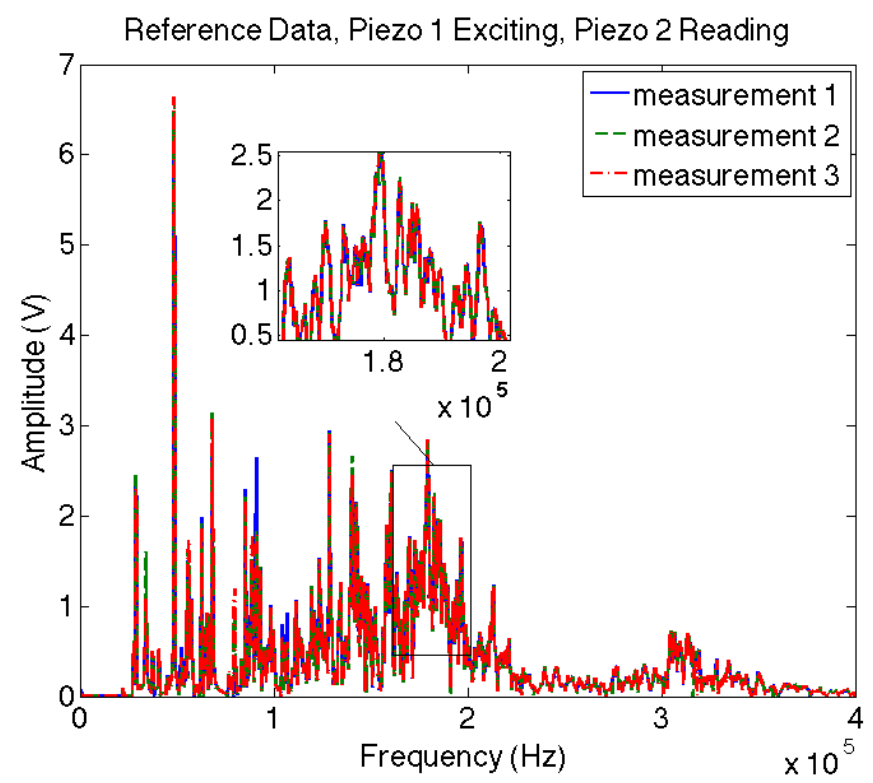

Figure 6-25 Reference data measured from piezo 2 when piezo 1 is exciting

The change in the spectrums due to the presence of damage is investigated. The experimentation set up is similar to the laser implementation procedure except the fact that the laser sensor was not used. Once one of the piezoelectric elements was dedicated to excitating, the remaining elements were used as sensors. This way five reference data sets were collected. Each of these data sets included the data from four piezo sensors. Figure 6-26 shows spectrums of damaged block. In order to show the changes more clearly the data is presented in a zoomed range $(110-150 \mathrm{kHz})$. 


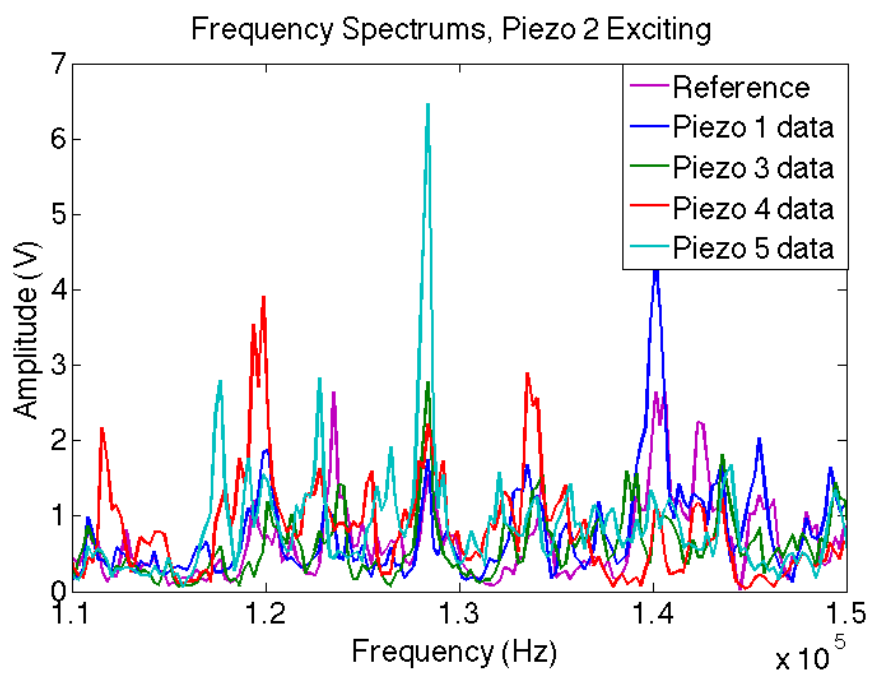

Figure 6-26 Frequency spectrums data measured after surface damage to block; piezo 2 exciting and piezo 1, 3, 4 and 5 sensing

In order to quantify the changes in the spectrums after introductin of milling slut, PbPPM uses the sum of square of differences (SSD) index shown in Table 6-5. This tabel reflects the change in SSD index calculated from piezoelectric-based measurements. 
Table 6-5 Sum of squared differences before and after damage

\begin{tabular}{lllllll}
\hline Exciter & & Piezo1 & Piezo2 & Piezo3 & Piezo4 & Piezo5 \\
\hline Piezo1 & Ref & & 0.47 & 0.62 & 14.22 & 11.17 \\
& Dmg & & 16.48 & 164.57 & 172.44 & 141.09 \\
\hline Piezo2 & Ref & 11.51 & & 13.03 & 17.18 & 7.63 \\
& Dmg & 226.33 & & 290.14 & 154.25 & 119.33 \\
\hline Piezo3 & Ref & 20.47 & 13.35 & & 4.05 & 7.08 \\
& Dmg & 211.70 & 301.36 & & 200.88 & 163.43 \\
\hline Piezo4 & Ref & 6.72 & 5.69 & 21.11 & & 3.60 \\
& Dmg & 136.99 & 175.03 & 159.89 & & 158.50 \\
\hline Piezo5 & Ref & 4.57 & 9.38 & 2.89 & 4.32 & \\
& Dmg & 125.78 & 101.63 & 110.95 & 226.18 & \\
\hline
\end{tabular}

The lowest SSD values were when piezo 4 and piezo 5 were exciting. Still these values are well above the uncertainty values and indicate the presence of damage.

A bar diagram of SSD values when piezo 3 was exciting is shown in Figure 6-27. SSDs have increased more in data from piezo 1 and 2 than data from piezo 4 and 5 . This could be due to the fact that in the former the exciter, sensor and damage are all located on the same plane. Also another observation is that the highest SSD is calculated in data from piezo 2. This could be interpreted due to the fact that the surface damage is located between the piezo 1 and 3. 


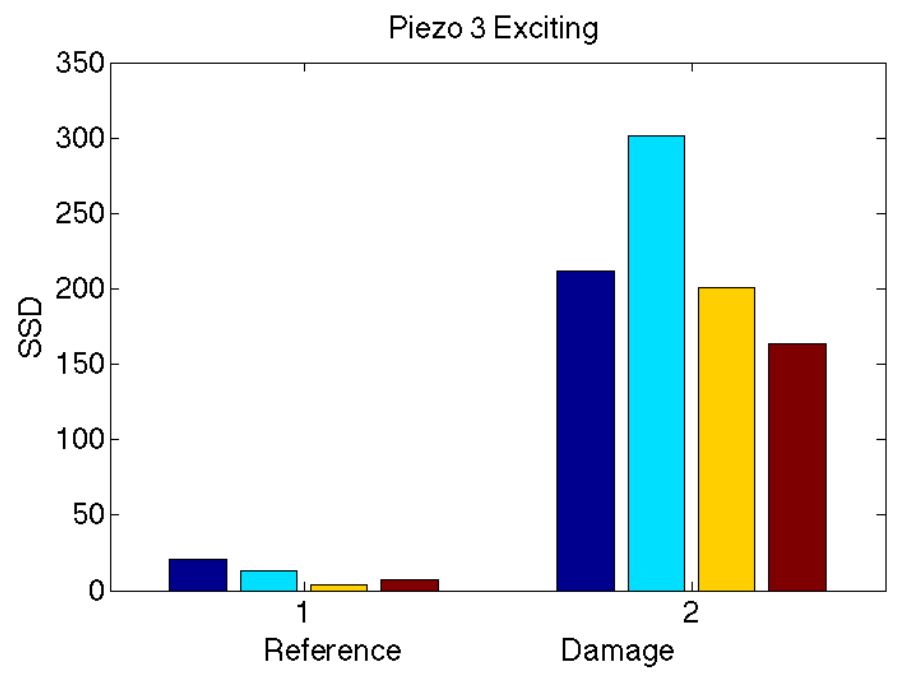

Figure 6-27 Bar diagram of sum of square of differences from laser data; piezo 3 exciting and piezo 1, 2, 4 and 5 reading

\subsubsection{Non-Contact Laser vibrometer-based implementation}

In the case of measurements from Laser vibrometer, data was collected from all six faces of the block. The sensing points were shown in Figure 6-24. Figure 6-28 shows the SSD values from the laser measurement scan points, as piezo 1 was exciting.

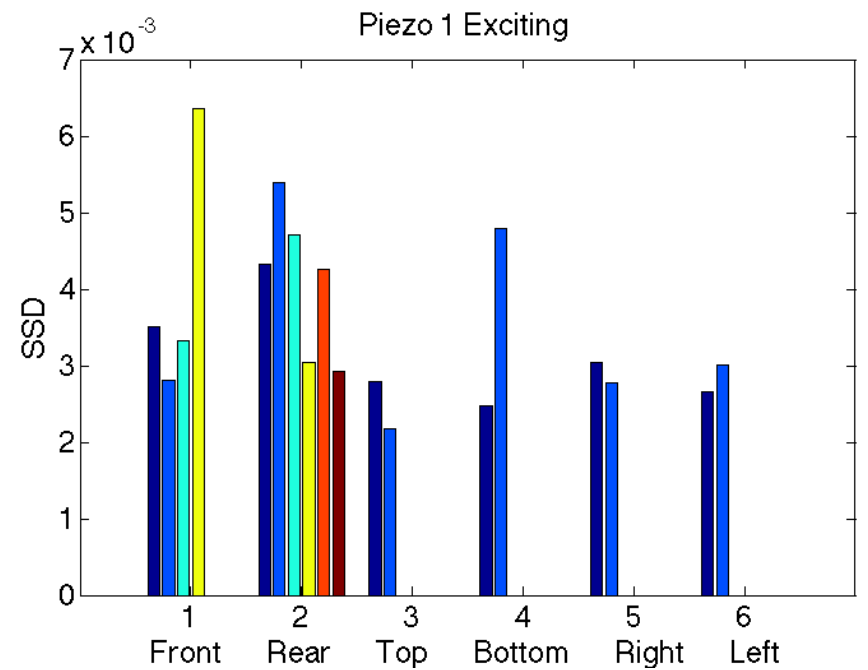

Figure 6-28 Sum of square of differences from laser data; piezo 1 exciting and laser vibrometer measuring data from scan points 


\subsubsection{DSP device-based implementation}

The monitoring frequency in the case of experiments with digital signal processing device was limited to $20-250 \mathrm{kHz}$. The frequency response function after the creation of surface damage on the block is shown in Figure 6-29.

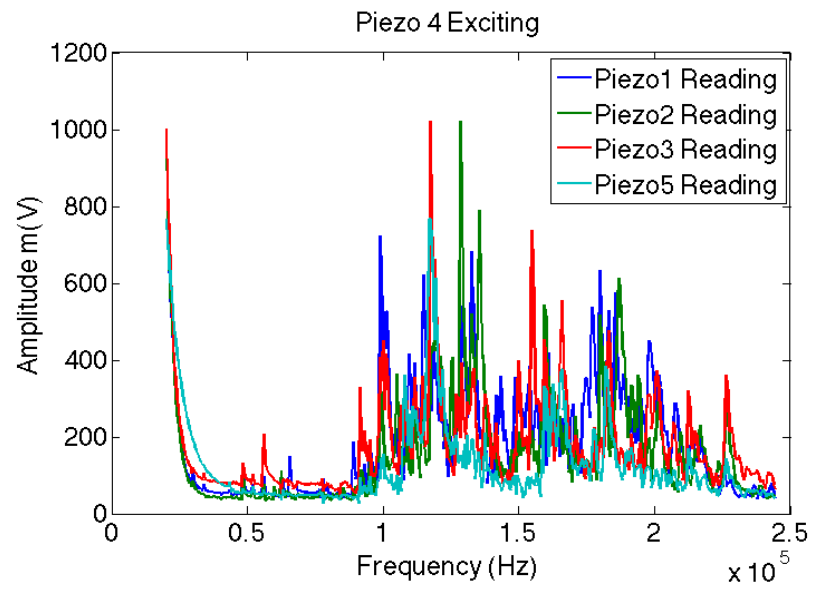

Figure 6-29 Frequency spectrums from DSP data; piezo 4 exciting piezo 1, 2, 3, 5 reading

The highest range of peaks is within $100-220 \mathrm{kHz}$. The sum of squares of differences for data from DSP device is shown in Figure 6-30.

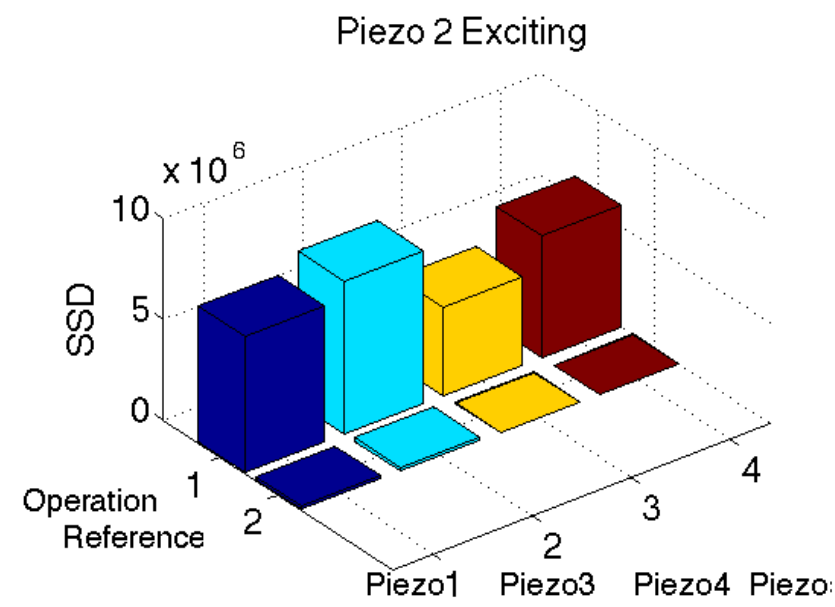

Figure 6-30 Sum of square of differences from DSP data; piezo 2 exciting and piezo 1, 2, 3 and 4 reading 


\subsubsection{Conclusion}

The experimentations are designed to evaluate the performance of PbPPM method in machining monitoring of multi-face three-dimensional objects.

Different scenarios where considered where the exciter, sensor and damage were not located in same planes. SuRE method was implemented using laboratory equipment's, laser scanning vibrometer (LSV) and an embedded low cost digital signalprocessing (DSP) device

All of the baseline spectrums overlapped before introducing any damage structure. As soon as the surface damage was introduced to the structure the spectrums changed for all measurement points on the block. the SSD values could discriminate the location of presence of damage only when the sensor and exciter where located in the plane of damage (If it is between the sensors or not was making a difference in SSD values). The lowest SSD values were the case of piezo 4 and piezo 5 exciting. Still these values are well above the uncertainty values and indicate the presence of damage. Although the laser scanning vibrometer measured signals where much weaker than those of piezos, there was a considerable increase in SSD values in presence of damage. The low cost embedded DSP board developed for the PbPPM method captured results very similar to those of expensive laboratory equipment. 


\section{RESULTS AND DISCUSSION}

In this chapter, the performance of the SuRE method is evaluated when a simple DSP, spectrum analyzer and scanning laser vibrometer were used in separate studies. Performances of Teager-Kaiser (TKA) [19,20], Goertzel [21], RMS and average of the positive readings algorithms will be discussed for estimating the characteristics of the piezo-structure combination.

The outline of this chapter is as follows: first, the performances of the amplitude estimation algorithms will be compared. These algorithms were considered for the DSP system. Next, the performance of the DSP system will be presented. Spectrum analyzer was used for inspecting composite coating. Finally, the performance of using the SuRE method with a scanning laser vibrometer will be discussed for detecting a loading point.

7.1. Investigation of Computational Efficiency and Validity of the Surface Response to Excitation Method

The Surface Response to Excitation (SuRE) method excites the surface of the structure with a piezoelectric element. The generated vibrations at critical points on the surface are measured with other piezoelectric elements, a laser vibrometer or other sensors. Then, the magnitude of the transfer function between the excitation and the sensory signal is monitored. The performance of the SuRE method was evaluated using a low-cost Digital Signal Processor (DSP) system, spectrum analyzer, and laser vibrometer. The accuracies of the Teager-Kaiser (TKA), Goertzel, RMS and average of the positive values algorithms were evaluated for magnitude estimation. The SuRE method was employed successfully for detecting compression loads by using the DSP system. 
Existence of composite coating was inspected using a spectrum analyzer. Finally, the method was used to detect localized loads on an aluminum plate with a laser vibrometer. To speed up analysis by the use of the incoming signal by the DSP, two methods were initially considered, the Teager-Kaiser (TKA) [20,22,23] and Goertzel [21] algorithms.

\subsubsection{Comparison of magnitude estimation methods}

First, a sweep sine wave was generated with 0.00001 second sampling interval between $2 \mathrm{kHz}$ and $35 \mathrm{kHz}$ range. There were 100 frequency components in the signal and 10 periods of the sine wave were created at each frequency. First, the amplitude of the signal was estimated by using Goertzel, RMS, average of the positive values and TKA algorithms, shown on the left of Figure 7-1. Goertzel and RMS algorithms gave very similar and most accurate estimations. The estimations of the average of the positive values algorithm were also very good. All the algorithms outside of the TKA knew the excitation frequency, worked with the data of 10 periods of the sine wave at each frequency and estimated only the amplitude. The TKA algorithm estimated the frequency and amplitude from only 3 samples. The estimation accuracy was lower compared to the others at these much difficult estimation conditions.

Second, the frequency response of a 1-DOF (Degree Of Freedom) system with 15 $\mathrm{kHz}$ natural frequencies and 0.01 damping ratio was simulated for the sweep sine wave. The Goertzel, RMS and average of positive values based algorithms made very similar estimations on the right side of Figure 7-1. The results showed the Goertzel gave the most accurate estimations. The estimations of the RMS and the average of the positive values 
algorithm were almost the same. The TKA algorithm results were not put in the plot since the accuracy of the method was not as good as the others.
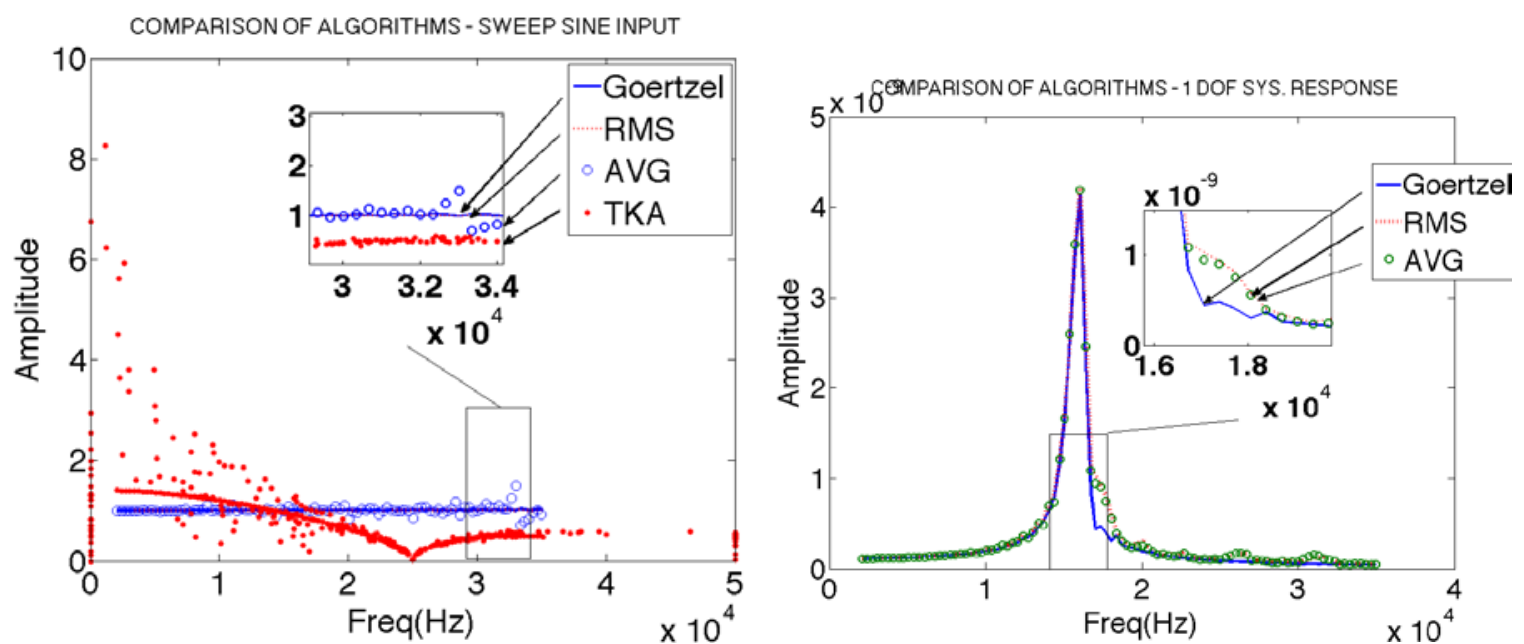

Figure 7-1 The frequency response estimation for a sweep sine wave signal of amplitude 1 (left), The frequency response estimation for a simulated 1-DOF system (right).

\subsubsection{Implementation of the SuRE method with a DSP system}

The Microstick-based DSP was programmed with the average of the positive values algorithms. It was used for detecting a compressive force applied to the middle of an aluminum beam.

The DSP took less than 5 seconds to scan the determined frequency interval and correctly identified the existence of the compressive force of a hydraulic crimper. The experiment was repeated twelve times. In the first four tests, there was no compressive force. The data was collected four more times with the compressive force applied. Later, the compressive force was released and data was taken again four times. The Microstick calculated the amplitudes at the scanned frequencies and uploaded them to a PC. The averages of four experiments at three different conditions are presented in Figure 7-2, 
left. The first case without any compressive force was used as reference and the sums of the squares of the differences were calculated at the twelve conditions (Figure 7-2, right). The magnitude characteristics of the initial and after-release cases were very similar to the reference and sums of the squares of the differences were very small. The sum of the squares of the difference increased drastically when the compressive force was applied. The performance of the DSP circuit was evaluated with and without compressive force for 1 hour. No wrong estimation was encountered.
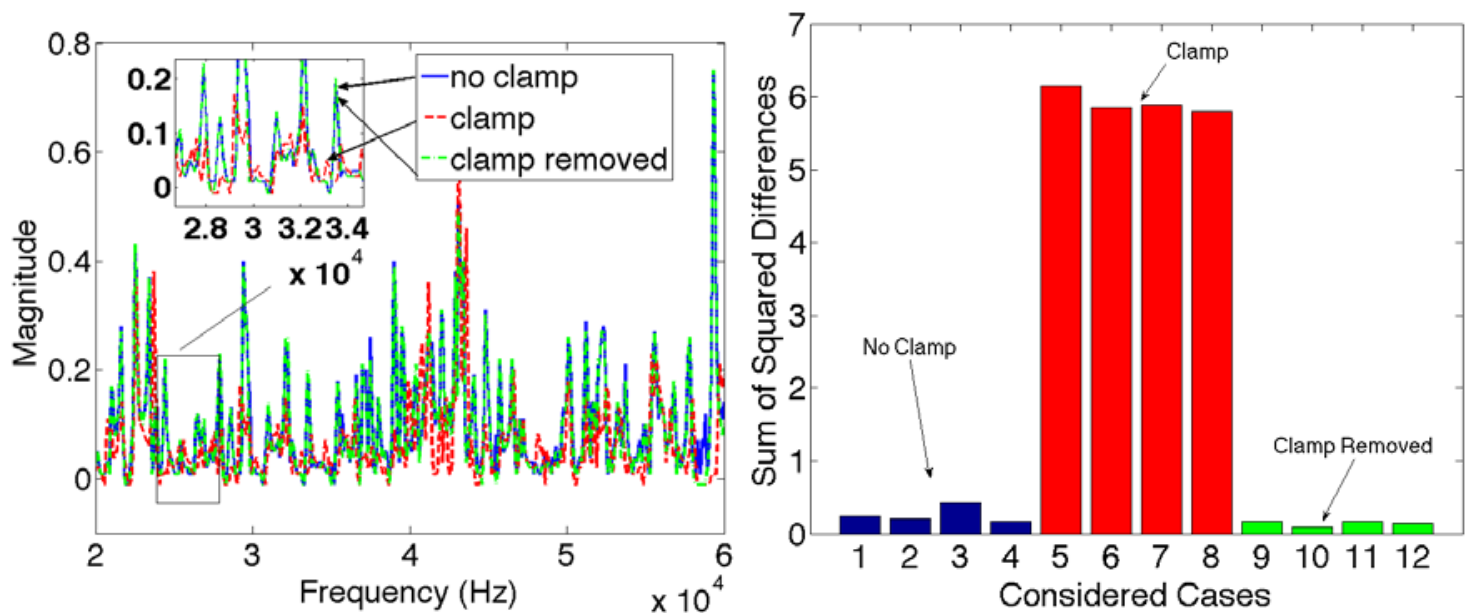

Figure 7-2 The average magnitude response of the of the tests at the initial condition (Data 13), with the load applied (Data 14), and with the load released (Data 15). The SSD values for the 12 test cases were also calculated (right).

\subsubsection{Inspection of composite coating by using the SuRE method}

An aluminum beam with $(41 \mathrm{~cm} \times 4 \mathrm{~cm} \times 0.16 \mathrm{~cm})$ dimensions was used in the experiments. A composite coating with approximately $0.32 \mathrm{~cm}$ thickness was applied to one side of the beam which has no piezoelectric elements, shown in Figure 7-3. 

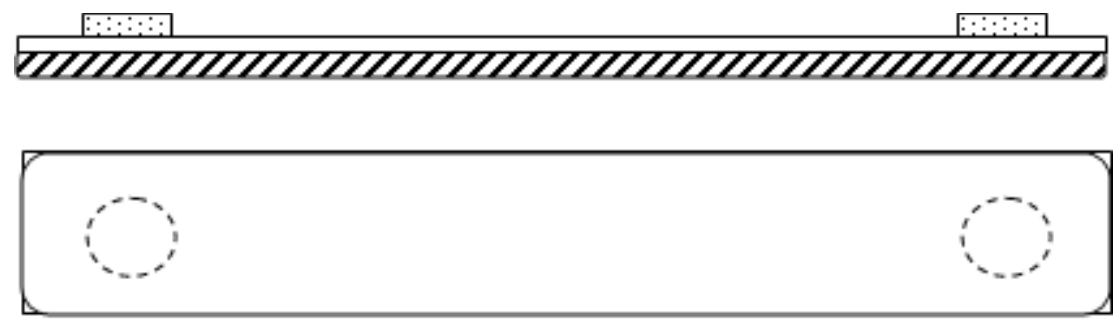
fiberglass

Figure 7-3 One side of the aluminum test beam surface was coated with a

The experiments were performed using the spectrum analyzer. The magnitudes of the transfer functions calculated with the SuRE method, and the sum of the squares of the differences before and after the coating are compared at six different frequency intervals in Figure 7-4. The six frequency intervals are $5 \mathrm{kHz}-16.7 \mathrm{kHz}, 16.7 \mathrm{kHz}-33.3 \mathrm{kHz}$, $33.3 \mathrm{kHz}-50$ kHz, 50 kHz - 66.6 kHz, 66.6 kHz - 83.3 kHz, 83.3 kHz - 100 kHz, from left to right.
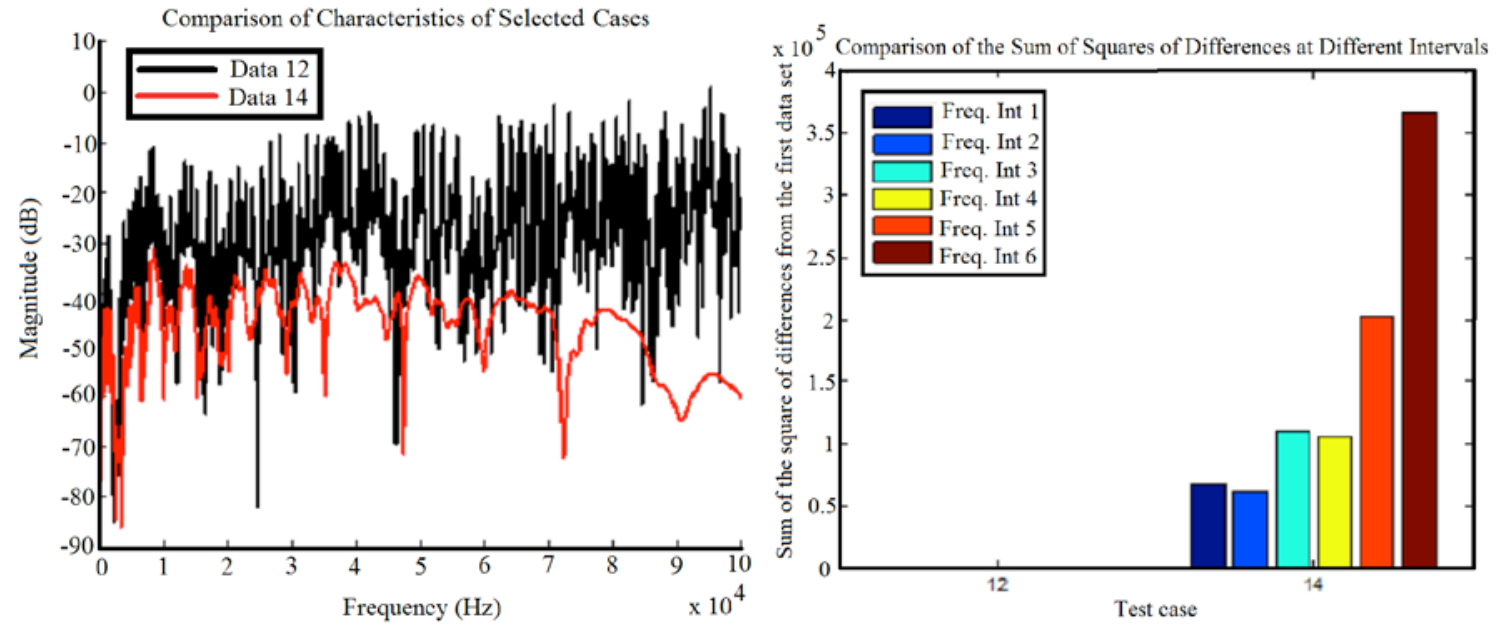

Figure 7-4 The estimated magnitude response (left) and the sum of the squares of the differences at 6 frequency intervals (right). Data 12 and 14 are without and with the coating respectively.

The coating process made significant change at all frequency bands. This observation indicated that the manufacturing quality or integrity of the composite coating may be monitored by using cheaper DSPs at sampling rates as low as $60 \mathrm{kHz}$. The 
change was five times significant at the $80 \mathrm{kHz}-100 \mathrm{kHz}$ band. The sensitivity and resolution is supposed to be better at this band. The results indicated that the SuRE method has similar characteristics and capabilities with the impedance method. The data collection and analysis may be significantly simplified by adding one piezoelectric element.

7.1.3.1. Implementation of the SuRE method by using scanning laser vibrometer:

An aluminum plate with a piezoelectric element bonded to the center was mounted in a wood frame, shown in Figure 7-5 (a). Single-point force was applied to the back of the plate by tightening a bolt. The piezoelectric element was excited with a sweep sine wave from $20 \mathrm{kHz}$ to $40 \mathrm{kHz}$. A PolyTech PSV-400 scanning laser vibrometer was used to measure the vibrations at selected grid points on the surface of the plate, similar to how the previous experiments used the second piezoelectric element. However, in this case, the laser could scan dozens of points of the entire surface and generate each point's frequency response.

The scanning laser vibrometer scanned a grid with $10 \times 10$ points covering an area of $6 " \times 11 "$ on the surface of the plate. This surface scan data obtained by the laser was then fed into Matlab software and contour plot was prepared from the sum of the squares of the differences of the spectrums Figure 7-5 (b). 


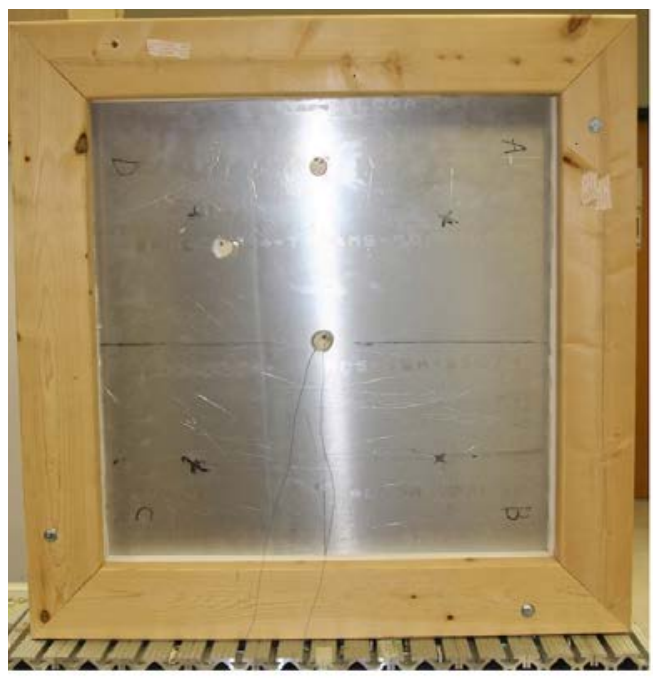

(a)

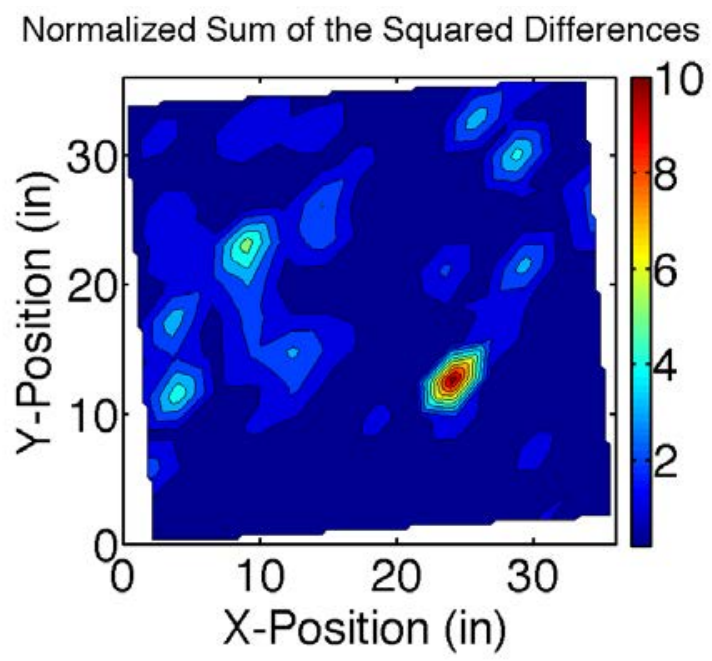

(b)

Figure 7-5 Various scans taken with the laser vibrometer looking for localized loads. The load was applied to the bottom right.

\subsection{Effectiveness of tested approaches}

7.2.1. Comparison of effectiveness of laser scanning vibrometer, DSP system and spectrum analyzer for implementing SuRE method

In order to compare the performances of each of the methods introduced for the implementing the SuRE method, the set up shown in the schematic of Figure 3-9 was used. The Non-destructive load experimentation was designed to compare the performance of PolyTech PSV400 laser scanning vibrometer, Stanford Systems spectrum analyzer and Mikrostick with dsPIC33F. Each device captured two initial reference scans. Then, for each of the six points shown in Figure 7-6(a), the data was captured two times; the first time after application of load and the second time after removing the load by releasing the clamp. In the case of laser vibrometer, the laser beam pointed to the location of monitoring PZT on the beam. 


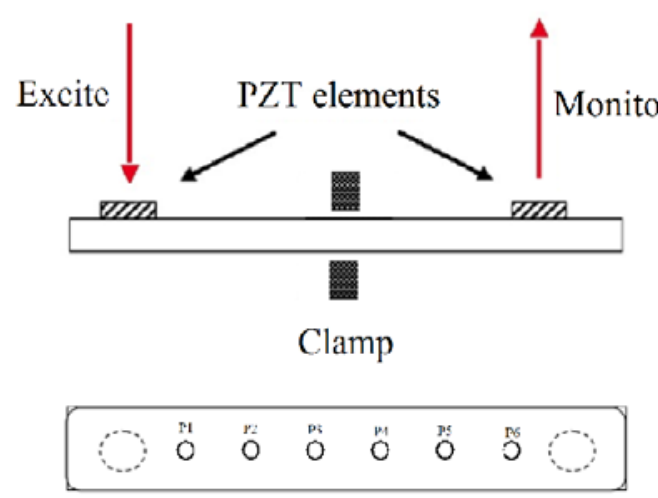

(a)

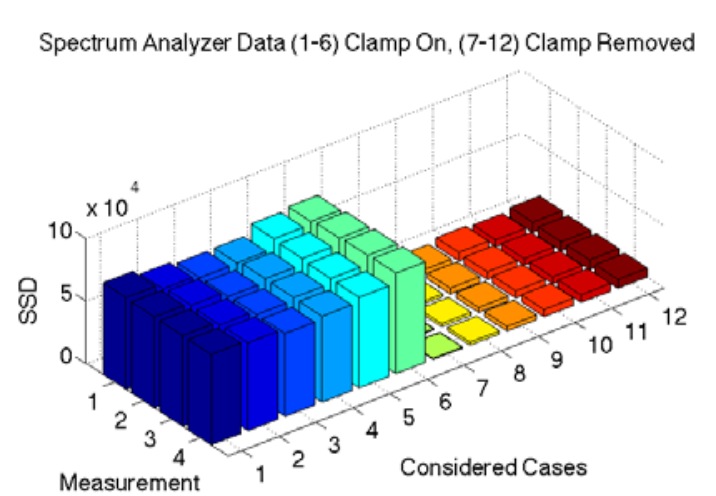

(b)

Figure 7-6 Comparison of sum of squares of differences, Column 1-6 is corresponding to clamp applying load on point P1-P6; Schematic of load tests for comparison (left), data from spectrum analyzer (right)

Three data sets were obtained corresponding to devices that were used. According to the SuRE method, sums of squares of differences of measured spectrums versus reference spectrums were calculated for all data sets. Figure 7-6 (b) compares the SSDs processed from laser data with the data from the spectrum analyzer. The columns 1-6 show the SSDs when the load applied to the points P1-P6. Columns 7-12 show the SSDs corresponding to removal of load from the points. Data were recorded four times at every step of experiment for the purpose of uncertainty analysis. Table 1 shows the normalized sum of squares of differences for the three experiments performed. The data from the spectrum analyzer is the most robust indicator of the change in the load condition of beam. In Figure 7-7, the sums of squares of differences are normalized respect to their maximum values. 


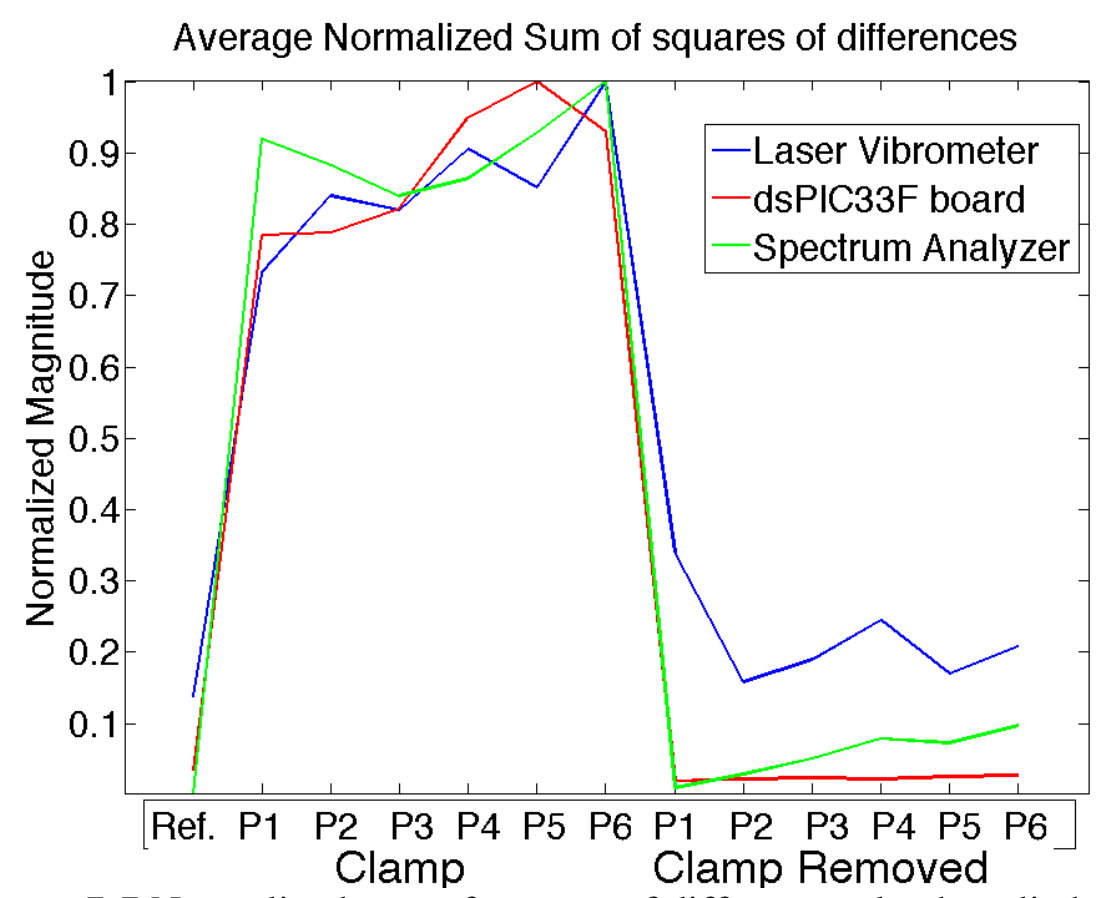

Figure 7-7 Normalized sum of squares of differences; load applied with the clamp to points 1-6 respectively (left P1-P6), Load removed from the point 1-6 (right P1-P6)

While the behaviors of dsPIC and spectrum analyzer don't show any pattern at all, the laser vibrometer shows a semi-consistent increase in normalized values. After the removal of load, the SSDs go back to their original reference values while, depending on the sensitivity of the devices, some small residues were left.

Table 7-1 NSSDs for Laser vibrometer (LSV), dsPIC33F (DSP), spectrum analyzer (SA)

\begin{tabular}{lllllll}
\hline Clamp & Point 1 & Point 2 & Point 3 & Point 4 & Point 5 & Point 6 \\
\hline LSV & 7.4164 & 8.5018 & 8.3006 & 9.1697 & 8.6205 & 10.1184 \\
\hline DSP & 33.8166 & 25.5027 & 35.4274 & 40.9369 & 43.0866 & 40.0547 \\
\hline SA & 766.9559 & 736.4150 & 700.0259 & 720.6376 & 774.3221 & 833.3652 \\
\hline Release & Point 1 & Point 2 & Point 3 & Point 4 & Point 5 & Point 6 \\
\hline LSV & 3.4392 & 1.6033 & 1.9178 & 2.4737 & 1.7179 & 2.1119 \\
\hline DSP & 0.8345 & 0.9309 & 1.0198 & 0.9443 & 1.0980 & 1.1881 \\
\hline SA & 7.7818 & 24.2918 & 42.1611 & 65.3319 & 60.2773 & 80.6466 \\
\hline
\end{tabular}




\subsubsection{Uncertainty of Measurements}

During the experimentation, the data in each step was measured four times. The root mean square (RMS) method was used to quantify the differences in spectrum measurements during similar test conditions and is presented in Table 7-2.

Table 7-2 Uncertainty in the form of percentage of variation in measurements calculated using the root mean square (RMS) method

\begin{tabular}{lccccccc}
\hline Measurement & Point1 & Point 2 & Point 3 & Point 4 & Point 5 & Point 6 & Average \\
& & & & & & & \\
\hline Laser & 2.9467 & 2.0153 & 3.2618 & 1.3587 & 2.8296 & 1.9090 & 2.3869 \\
\hline dsPIC board & 2.4742 & 2.6430 & 1.7761 & 1.6582 & 1.2519 & 1.6738 & 1.9129 \\
& & & & & & & \\
\hline Spectrum Ana & 1.1547 & 1.5462 & 0.7067 & 1.8028 & 1.7843 & 2.0536 & 1.5080 \\
\hline
\end{tabular}




\section{CONCLUSION}

The Surface Response to Excitation (SuRE) method was developed as an alternative to the electromechanical impedance method in SHM applications. The purpose of this study was to implement this method in metal cutting applications with a low-cost hardware. The reliability of the method was evaluated by detecting applied force on plates by using a laser scanning vibrometer (LSV). The Part-Based Process Performance Method (PBPPM) was tested on metal and composite plates. Digital Signal Processor (DSP)-based hardware was developed to excite the workpiece with a series of sine waves and obtain the spectral characteristics of the monitored point.

Finite element analysis was performed by COMSOL software, which simulated the SuRE method via a frequency domain study. FEA clearly revealed the performance mechanism of high-frequency surface waves during interaction with structural defects. Characteristics of solution and the behavior of spectrums of probe points adjacent to simulated milling, drilling and cutting operations not only revealed the behavior of highfrequency surface waves during interaction with cutting edges but also prospected the promising potential of the SuRE method for part-based manufacturing process monitoring.

The LSV monitored the surface oscillations successfully. The LSV could scan the surface vibrations at multiple points on the surface of a plate without attaching any piezoelectric elements. The location of the external force could be estimated from the spectral deviations at the grid points. 
The integrity of a robotic arm was examined remotely via a scanning laser vibrometer (SLV) in order to detect loose bolts. The study shows that the SuRE method was capable of detecting the presence and location of multiple scenarios of loosening bolts using only one PZT element on a complex structure.

A low-cost DSP system was developed for the implementing the SuRE method. Two neural network (the Levenberg-Marquardt and the scaled conjugated gradient) algorithms were used to correlate the spectral characteristics with the location of an applied force on the surface of a beam. This approach required attachment of only one piezoelectric element to the surface of the plate.

The sums of the squares of differences (SSDs) on a grid were calculated after the machining operations were completed. Three basic metal cutting operations, cutting, drilling and milling, were considered. The 2D color maps revealed the location of the machining operations.

The sensitivity of the SuRE method was tested when the three basic parameters of the slot, namely length, depth and width, were changed. The sensitivity was very good when the length and width were changed. It was minimum when the depth changed.

The SuRE method was originally developed for thin plates. Generally, large plates are used in the experiments to avoid the waves coming from the boundary surfaces (edge of the plates). The SuRE method was used for machining processes monitoring when the cutting operations were performed on a small thick block. The machining operation was easily detected from the signals of the sensors located at different surfaces of the block. The low-cost embedded DSP board quickly obtained the SSDs with the accuracy of expensive laboratory equipment, such as spectrum analyzers. 
The developed DSP-based device reduced the experimental time and cost of implementing the SuRE method. The developed hardware is capable of working with non-contact sensors.

A through comparison of sensors, signal processing and implementation hardware showed that the SuRE method is very flexible and reliable in both SHM and PbPPM applications. It may be implemented by using various excitation, measurement (contact or non-contact) and analysis methods. Using multiple sensors or remotely scanning with a scanning laser vibrometer may estimate the location of the defect. Spectrum analyzers and even a simple DSP may complete the analysis of the data coming from the transducers. 


\section{REFERENCES}

1 Giurgiutiu, V. (2008). Structural Health Monitoring with Piezoelectric Wafer Active Sensors. London: Academic Press.

2 Farrar, C. R., Hemez, F. M., Shunk, D. D., Stinemates, D. W., Nadler, B. R., \& Czarnecki, J. J. (2004). A review of structural health monitoring literature: 1996-2001 (p. 303). Los Alamos, NM: Los Alamos National Laboratory.

3 Korla, S. (2011). Inspection of parts with complex geometry and welds with structural health monitoring techniques. Ph.D. Dissertation, Florida International University, Miami, FL.

4 Park, G., Cudney, H. H., \& Inman, D. J. (2000). An integrated health monitoring technique using structural impedance sensors. Journal of Intelligent Material Systems and Structures, 11(6), 448-455.

5 Park, G., Sohn, H., Farrar, C. R., and Inman, D. J. (2003). Overview of Piezoelectric Impedance-Based Health Monitoring and Path Forward, The Shock and Vibration Digest, 35(6), 451-463.

6 Wang, X., \& Tansel, I. N. (2007). Modeling the Propagation of Lamb Waves using a Genetic Algorithm and S-transformation. Structural Health Monitoring, 6(1), 25-37.

7 Lamb, H. (1993). Hydrodynamics. Cambridge university press.

8 Strutt, J. W., \& Rayleigh, L. (1885). On waves propagated along the plane surface of an elastic solid. Proceedings of the London Mathematical Society, 17, 4-1.

9 Worlton, D. C. (1961). Experimental confirmation of Lamb waves at megacycle frequencies. Journal of Applied Physics, 32(6), 967-971.

10 Mindlin, R. D. (1951). Influence of rotary inertia and shear on flexural motions of isotropic elastic plates.

11 Amirkulova, F. A. (2011). Dispersion relations for elastic waves in plates and rods (Doctoral dissertation, Rutgers University-Graduate School-New Brunswick).

12 Pardo de Vera, C., and James, L. (1997). Embedded Self-Sensing Piezoelectric for Damage Detection, IWSHM 445-455. Proceedings of the International Workshop on Structural Health Monitoring, Stanford University, CA. 
13 Peairs, D.M., Park, G., and Inman, D.J. (2004). Improving Accessibility of the Impedance-Based Structural Health Monitoring Method, Journal of Intelligent Material Systems and Structures, 15(2), 129-139.

14 Giurgiutiu, V., and Xu, B. (2004). Development of a Field-Portable Small-Size Impedance Analyzer for Structural Health Monitoring using the Electromechanical Impedance Technique, SPIE's 11th Annual International Symposium on SmartStructures and Materials and 9th Annual International Symposium on NDE for Health Monitoring and Diagnostics. San Diego, CA.

$15 \mathrm{Xu}$, B., and Giurgiutiu, V. (2005). A Low-Cost and Field Portable Electromechanical (E/M) Impedance Analyzer for Active Structural Health Monitoring, IWSHM 634-644. Proceedings of the 5th International Workshop on Structural Health Monitoring. Stanford University, CA.

16 Li, M., Demetgul, M., Li, X., and Tansel, I.N. (2009). Development of Effective Structural Health Monitoring Strategies By Using Self Organized Map and Index Based Reasoning, IWSHM 561-568. From System Integration to Autonomous Systems, 7th International Workshop of Structural Health Monitoring, Stanford University, Stanford, CA, USA.

17 Bhalla, S., Gupta, A., Bansal, S., and Garg, T. (2009). Ultra Low-cost Adaptations of Electro-mechanical Impedance Technique for Structural Health Monitoring, Journal of Intelligent Material Systems and Structures, 20(8), 991999.

18 Panigrahi, R., Bhalla, S., and Gupta, A. (2010). A Low-cost variant of electromechanical impedance (EMI) technique for Structural Health Monitoring, Experimental Techniques, 34(2), 25-29.

19 Kaiser, J.F. (1990). On a simple algorithm to calculate the 'energy' of a signal, ICASSP-90 381-384. International Conference on Acoustics, Speech, and Signal Processing.

20 Pai, F.P. (2010). Online tracking of instantaneous frequency and amplitude of dynamical system response, Mechanical Systems and Signal Processing, 24, 1007-1024.

21 Goertzel, G. (1958). An algorithm for the evaluation of finite trigonomentric series, The American Mathematical Monthly, 65(1), 34-35.

22 Vakman, D. (1996). On the analytic signal, the Teager-Kaiser energy algorithm, and other methods for defining amplitude and frequencyk, IEEE Transactions on Signal Processing, 44, 791-797. 
23 Maragos, P., Kaiser, J.F., and Quatieri, T.F. (2011). Energy separation in signal modulations with application to speech analysis, IEEE Transactions on Signal Processing, 41.

24 Efficiently detecting a frequency using a Goertzel filter, Networking Embedded Systems [Internet]. [Updated 2011 August 25, 2011, cited 2012 December 29]; Available from: http://netwerkt.wordpress.com/2011/08/25/goertzel-filter/.

25 Bissell, C.C and Chapman, D.A. (1992). Digital signal transmission, Cambridge University Press.

26 Ou, J., \& Li, H. (2010). Structural health monitoring in mainland China: review and future trends. Structural Health Monitoring, 9(3), 219-231.

27 Martowicz, A., Rosiek, M., Stepinski, T., Uhl, T., \& Staszewski, W. Electromechanical Impedance Method. Advanced Structural Damage Detection: From Theory to Engineering Applications, 141-176.

28 Giurgiutiu, V. (2007). Structural health monitoring: with piezoelectric wafer active sensors. Academic Press.

29 Tansel, I. N., Grisso, B. L., Singh, G., Singh, G., Korla, S., \& Salvino, L. W. (2011). Health Monitoring of Aluminum Weldings with the Surface Response to Excitation (SuRE) Approach. NAVAL SURFACE WARFARE CENTER CARDEROCK DIV BETHESDA MD STRUCTURES AND COMPOSITES DIV.

30 Li, M., Li, X., Tansel, I. N., \& Demetgul, M. (2009, September). Development of Effective Structural Health Monitoring Strategies Using Self Organized Map and Index Based Reasoning. In Structural Health Monitoring (Proceedings of the 8th International Workshop on Structural Health Monitoring (IWSHM 2009)) Edited by Fu-Kuo Chang, DEStech Publications (pp. 561-568).

31 Tansel, I. N., Grisso, B. L., Singh, G., Singh, G., Korla, S., Duran, R., \& Salvino, L. W. (2011). Wear estimation by testing the elastic behavior of tool surface. International Journal of Machine Tools and Manufacture, 51(10), 745-752.

32 Fekrmandi, H., Rojas, J., Campbell, J., Tansel, I. N., Kaya, B., Taskin, S. (2014). Inspection of the Integrity of a Multi-Bolt Robotic Arm Using a Scanning Laser Vibrometer and Implementing the Surface Response to Excitation Method (SuRE), International Journal of Prognostics and Health Management, 5 (1): 110 .

33 Monitoring The Health of a Beam Remotely by Using Scanning Laser Vibrometer," H. Fekrmandi, J. Rojas, M. Wolff, I. N. Tansel, S. Gonzalez, B. 
Uragun, (2013) ASME Early Career Technical Conference Proceedings, ECTC 2013, 12: 302-306.

34 Implementing the Surface Response to Excitation Method (SuRE) With NonContact Sensor at Hard to Reach Locations, (2013) "S. Gonzalez, J. Rojas, H. Fekrmandi, I. N. Tansel, B. Uragun”, Structural Health Monitoring 2013, a Roadmap to Intelligent Structures: Proceedings of the Ninth International Workshop, September 10-13, , Stanford University

35 Rehorn, A. G., Jiang, J., \& Orban, P. E. (2005). State-of-the-art methods and results in tool condition monitoring: a review. The International Journal of Advanced Manufacturing Technology, 26(7-8), 693-710.

36 Tansel, I. N., \& Yapici, A. (2013). Part based process performance monitoring (PbPPM). Journal of Manufacturing Processes, 15(3), 329-337.

37 Giurgiutiu, V., \& Rogers, C. A. (1999). Modeling of the electro-mechanical (E/M) impedance response of a damaged composite beam. Adaptive Struct. Mater. Syst, 87, 39-46.

38 Zagrai, A. N., \& Giurgiutiu, V. (2001). Electro-mechanical impedance method for crack detection in thin plates. Journal of Intelligent Material Systems and Structures, 12(10), 709-718.

39 Liu, W., \& Giurgiutiu, V. (2007, January). Finite element simulation of piezoelectric wafer active sensors based structural health monitoring. In ASME 2007 International Mechanical Engineering Congress and Exposition (pp. 715726). American Society of Mechanical Engineers.

40 Yang, Y., Lim, Y. Y., \& Soh, C. K. (2008). Practical issues related to the application of the electromechanical impedance technique in the structural health monitoring of civil structures: I. Experiment. Smart Materials and Structures, 17(3), 035008.

41 Raghavan, A., \& Cesnik, C. E. (2007). Review of guided wave structural health monitoring. Shock and Vibration Digest, 39(2), 91-116.

42 Su, Z., Ye, L., \& Lu, Y. (2006). Guided Lamb waves for identification of damage in composite structures: A review. Journal of sound and vibration, 295(3), 753-780.

43 Lee, B. C., \& Staszewski, W. J. (2003). Modelling of Lamb waves for damage detection in metallic structures: Part I. Wave propagation. Smart Materials and Structures, 12(5), 804. 
44 Lee, B. C., \& Staszewski, W. J. (2003). Modelling of Lamb waves for damage detection in metallic structures: Part II. Wave interactions with damage. Smart Materials and Structures, 12(5), 815.

45 Staszewski, W., Boller, C., \& Tomlinson, G. R. (Eds.). (2004). Health monitoring of aerospace structures: smart sensor technologies and signal processing. Wiley. com.

46 Giurgiutiu, V., Bao, J., \& Zhao, W. (2003). Piezoelectric wafer active sensor embedded ultrasonics in beams and plates. Experimental Mechanics, 43(4), 428449.

47 Tansel, I. N., Singh, G., Korla, S., Grisso, B. L., Salvino, L. W., \& Uragun, B. (2011, June). Monitoring the integrity of machine assemblies by using surface response to excitation (SuRE) approach. In Recent Advances in Space Technologies (RAST), 2011 5th International Conference on (pp. 64-67). IEEE.

48 Doebling, S. W., Farrar, C. R., Prime, M. B., \& Shevitz, D. W. (1996). Damage identification and health monitoring of structural and mechanical systems from changes in their vibration characteristics: a literature review (No. LA--13070MS). Los Alamos National Lab., NM (United States).

49 Sharma, V. K. (2009). Laser doppler vibrometer for efficient structural health monitoring. PhD thesis, Georgia Institute of Technology, 2008.

50 Staszewski, W. J., Lee, B. C., Mallet, L., \& Scarpa, F. (2004). Structural health monitoring using scanning laser vibrometry: I. Lamb wave sensing. Smart Materials and Structures, 13(2), 251.

51 Mallet, L., Lee, B. C., Staszewski, W. J., \& Scarpa, F. (2004). Structural health monitoring using scanning laser vibrometry: II. Lamb waves for damage detection. Smart Materials and Structures, 13(2), 261.

52 Leong, W. H., Staszewski, W. J., Lee, B. C., \& Scarpa, F. (2005). Structural health monitoring using scanning laser vibrometry: III. Lamb waves for fatigue crack detection. Smart Materials and Structures, 14(6), 1387.

53 Martarelli, M. (2001). Exploiting the laser scanning facility for vibration measurements (Doctoral dissertation, University of London).

54 Fink, M. (1992). Time reversal of ultrasonic fields. I. Basic principles. Ultrasonics, Ferroelectrics and Frequency Control, IEEE Transactions on, 39(5), 555-566. 
55 Wu, F., Thomas, J. L., \& Fink, M. (1992). Time reversal of ultrasonic fields. Il. Experimental results. Ultrasonics, Ferroelectrics and Frequency Control, IEEE Transactions on, 39(5), 567-578.

56 Sohn, H., Park,H., Law,K.H., and Farrar, C.R., (2004), Instantaneous Damage Detection using Time Reversal Process, 15th International Conference on Adaptive Structures and Technologies, October 2004.

57 Todd, M. D., Nichols, J. M., Nichols, C. J., \& Virgin, L. N. (2004). An assessment of modal property effectiveness in detecting bolted joint degradation: theory and experiment. Journal of sound and vibration, 275(3), 1113-1126.

58 Yan, W., \& Chen, W. Q. (2010). Structural health monitoring using highfrequency electromechanical impedance signatures. Advances in Civil Engineering, 2010.

59 Annamdas, V. G., \& Radhika, M. A. (2013). Electromechanical impedance of piezoelectric transducers for monitoring metallic and non-metallic structures: A review of wired, wireless and energy-harvesting methods. Journal of Intelligent Material Systems and Structures, 24(9), 1021-1042.

60 Ritdumrongkul, S., Abe, M., Fujino, Y., \& Miyashita, T. (2004). Quantitative health monitoring of bolted joints using a piezoceramic actuator-sensor. Smart Materials and Structures, 13(1), 20.

61 Chakraborty, D., Kovvali, N., Wei, J., Papandreou-Suppappola, A., Cochran, D., \& Chattopadhyay, A. (2009). Damage classification structural health monitoring in bolted structures using time-frequency techniques. Journal of Intelligent Material Systems and Structures, 20(11), 1289-1305.

62 Liang, C., Sun, F. P., \& Rogers, C. A. (1994). Coupled electro-mechanical analysis of adaptive material systems-determination of the actuator power consumption and system energy transfer. Journal of Intelligent Material Systems and Structures, 5(1), 12-20.

63 Arritt, B. J., Buckley, S. J., Ganley, J. M., Welsh, J. S., Henderson, B. K., Lyall, M. E., ... \& Roopnarine, R. (2008, March). Development of a satellite structural architecture for operationally responsive space. In The 15th International Symposium on: Smart Structures and Materials \& Nondestructive Evaluation and Health Monitoring (pp. 69300I-69300I). International Society for Optics and Photonics.

64 Bhalla, S., Naidu, A. S., \& Soh, C. K. (2003, October). Influence of structureactuator interactions and temperature on piezoelectric mechatronic signatures for 
NDE. In Smart Materials, Structures, and Systems (pp. 263-269). International Society for Optics and Photonics.Biographies.

65 Scheffer, C., \& Girdhar, P. (2004). Practical machinery vibration analysis and predictive maintenance. Access Online via Elsevier.

66 Giurgiutiu, Victor, Andrei Zagrai, and Jing Jing Bao. "Piezoelectric wafer embedded active sensors for aging aircraft structural health monitoring." Structural Health Monitoring 1.1 (2002): 41-61.

67 Staszewski, W. J., S. Mahzan, and R. Traynor. "Health monitoring of aerospace composite structures-Active and passive approach." Composites Science and Technology 69.11 (2009): 1678-1685.

68 Annamdas, Venu Gopal Madhav, and Chee Kiong Soh. "Application of electromechanical impedance technique for engineering structures: review and future issues." Journal of Intelligent material systems and structures 21.1 (2010): 41-59.

69 M. Li, X. Li, I.N. Tansel, M. Demetgul, "Development of Effective Structural Health Monitoring Strategies Using Self Organized Map and Index Based Reasoning", IWSHM pp. 561-568. Proceedings of the International Workshop on Structural Health Monitoring, Stanford University, CA, 2009.

70 Tansel, Ibrahim N., Javier Rojas, and Belamir Uragun. "Localization of problems with Surface Response to Excitation method." Recent Advances in Space Technologies (RAST), 2013 6th International Conference on. IEEE, 2013.

71 Fekrmandi, Hadi, et al. "Investigation of the Computational Efficiency and Validity of the Surface Response to Excitation Method." Measurement (2014).

72 Kosko, Bart. Neural Networks and Fuzzy Systems: A Dynamical Systems Approach to Machine Intelligence/Book and Disk. Vol. 1. Prentice hall, 1992.

73 Hagan, Martin T., and Mohammad B. Menhaj. "Training feedforward networks with the Marquardt algorithm." Neural Networks, IEEE Transactions on 5.6 (1994): 989-993.

74 Møller, Martin Fodslette. "A scaled conjugate gradient algorithm for fast supervised learning." Neural networks 6.4 (1993): 525-533.

75 Liang, S. Y., Hecker, R. L., \& Landers, R. G. (2002, January). Machining Process Monitoring and Control: The State-of-the-Art. In ASME 2002 International Mechanical Engineering Congress and Exposition (pp. 599-610). American Society of Mechanical Engineers. 
76 Teti, Roberto, et al. "Advanced monitoring of machining operations." CIRP Annals-Manufacturing Technology 59.2 (2010): 717-739.

77 Kalvoda, Tomas, and Yean-Ren Hwang. "A cutter tool monitoring in machining process using Hilbert-Huang transform." International Journal of Machine Tools and Manufacture 50.5 (2010): 495-501.

78 Dutta, S., Pal, S. K., \& Sen, R. (2014). Digital Image Processing in Machining. In Modern Mechanical Engineering (pp. 367-410). Springer Berlin Heidelberg.

79 Snr, Dimla, and E. Dimla. "Sensor signals for tool-wear monitoring in metal cutting operations - a review of methods." International Journal of Machine Tools and Manufacture 40.8 (2000): 1073-1098.

80 Xiao, C. W., Cheng, K., Ding, H., \& Chen, S. J. (2013, October). Design and analysis of a self-sensing smart cutting tool integrated piezoelectric films for cutting force monitoring in ultra-precision machining. In Piezoelectricity, Acoustic Waves and Device Applications (SPAWDA), 2013 Symposium on (pp. 1-4). IEEE.

81 Liao, Z. R., Gao, D., \& Lu, Y. (2014, August). Cutting Force Analysis in Tool Condition Monitoring of Difficult to Cut Materials. In Materials Science Forum (Vol. 800, pp. 175-179).

82 Lu, P., \& Chou, Y. K. (2013). Analysis of acoustic emission signal evolution for monitoring diamond-coated tool delamination wear in machining. Machining and Machine-tools: Research and Development, 1.

83 Sun, J., Wong, Y. S., Hong, G. S., \& Rahman, M. (2012). Feature analysis in tool condition monitoring: a case study in titanium machining. International Journal of Computer Applications in Technology, 45(2), 177-185.

84 Dinakaran, D., Sampathkumar, S., Sivashanmugam, N. (2009). An experimental investigation on monitoring of crater wear in turning using ultrasonic technique. International Journal of Machine Tools and Manufacture, 49(15), 1234-1237

85 Zhao, F., Mei, X., Du, Z., Tao, T., \& Jiang, G. (2012). Online Evaluation Method of Machining Precision Based on Built in Signal Testing Technology. Procedia CIRP, 3, 144-148.

86 Tangjitsitcharoen, S., Rungruang, C., \& Pongsathornwiwat, N. (2011). Advanced monitoring of tool wear and cutting states in CNC turning process by utilizing sensor fusion. Advanced Materials Research, 189, 377-384. 
87 Zhu, Kunpeng, Yoke San Wong, and Geok Soon Hong. "Wavelet analysis of sensor signals for tool condition monitoring: a review and some new results." International Journal of Machine Tools and Manufacture 49.7 (2009): 537-553.

88 Xiao, W., Zi, Y., Chen, B., Li, B., \& He, Z. (2014). A novel approach to machining condition monitoring of deep hole boring. International Journal of Machine Tools and Manufacture, 77, 27-33.

89 Kalvoda, Tomas, and Yean-Ren Hwang. "Analysis of signals for monitoring of nonlinear and non-stationary machining processes." Sensors and Actuators A: Physical 161.1 (2010): 39-45.

$90 \mathrm{Gu}$, Shuxin, Jun Ni, and Jingxia Yuan. "Non-stationary signal analysis and transient machining process condition monitoring." International Journal of Machine Tools and Manufacture 42.1 (2002): 41-51.

91 Kang, Myeong Chang, Jeong Suk Kim, and Jeon Ha Kim. "A monitoring technique using a multi-sensor in high speed machining." Journal of Materials Processing Technology 113.1 (2001): 331-336.

92 Haber, Rodolfo E., et al. "An investigation of tool-wear monitoring in a highspeed machining process." Sensors and Actuators A: Physical 116.3 (2004): 539545.

93 Kalvoda, T., Hwang, Y. R. (2010). A cutter tool monitoring in machining process using Hilbert-Huang transform. International Journal of Machine Tools and Manufacture, 50(5), 495-501.

94 Lu, M. C., Wan, B. S. (2013). Study of high-frequency sound signals for tool wear monitoring in micro-milling. The International Journal of Advanced Manufacturing Technology, 66(9-12), 1785-1792

95 Nikranjbar, A., Atai, A. A. (2013). Online model-based milling process condition monitoring. International Journal of Mechatronics and Manufacturing Systems, 6(2), 195-212. 4.

96 4. Hase, A., Wada, M., Koga, T., Mishina, H. (2014). The relationship between acoustic emission signals and cutting phenomena in turning process. The International Journal of Advanced Manufacturing Technology, 70(5-8), 9479555.

97 Lamraoui, M., Thomas, M., El Badaoui, M., Girardin, F. (2014). Indicators for monitoring chatter in milling based on instantaneous angular speeds. Mechanical Systems and Signal Processing, 44(1), 72-85 7. 
98 Hsieh, W. H., Lu, M. C., Chiou, S. J. (2012). Application of backpropagation neural network for spindle vibration-based tool wear monitoring in micromilling. The International Journal of Advanced Manufacturing Technology, 61(1-4), 53-61 8 .

99 u, F., uperl, U. (2011). Real-time cutting tool condition monitoring in milling. Strojniki vestnik-Journal of Mechanical Engineering, 57(2), 142-150 9.

100 Tangjitsitcharoen, S., Saksri, T., Ratanakuakangwan, S. (2013). Advance in chatter detection in ball end milling process by utilizing wavelet transform. Journal of Intelligent Manufacturing, 1-15 10.

101 Cao, H., Lei, Y., He, Z. (2013). Chatter identification in end milling process using wavelet packets and HilbertHuang transform. International Journal of Machine Tools and Manufacture, 69, 11-19. 11.

102 El Ouafi, A., Barka, N. (2014). An ANN Based Multi-Sensor Integration Approach for in-Process Monitoring of Product Quality in Turning Operations. Journal of Automation and Control Engineering Vol, 2(3) 13.

103 Brecher, C., Quintana, G., Rudolf, T., Ciurana, J. (2011). Use of NC kernel data for surface roughness monitoring in milling operations. The International Journal of Advanced Manufacturing Technology, 53(9-12), 953-962 14.

104 Huang, P. B. (2014). An intelligent neural-fuzzy model for an in-process surface roughness monitoring system in end milling operations. Journal of Intelligent Manufacturing, 1-12 15.

105 Marinescu, I., Axinte, D. A. (2011). An automated monitoring solution for avoiding an increased number of surface anomalies during milling of aerospace alloys. International

106 Journal of Machine Tools and Manufacture, 51(4), 349-357

Garcia-Romeu, M. L., Ciurana, J. (2011). Surface roughness monitoring application based on artificial neural networks for ball-end milling operations. Journal of Intelligent Manufacturing, 22(4), 607-617 17.

107 Bisu, C. F., Zapciu, M., Cahuc, O., Grard, A., Anica, M. (2012). Envelope dynamic analysis: a new approach for milling process monitoring. The International Journal of Advanced Manufacturing Technology, 62(5-8), 47148618.

108 Sun, F. _., Chaudhry, Z., Liang, C., \& Rogers, C. A. (1995). Truss structure integrity identification using PZT sensor-actuator. Journal of Intelligent material systems and structures, 6(1), 134-139. 
109 Chaudhry, Z. A., Joseph, T., Sun, F. P., \& Rogers, C. A. (1995, May). Local-area health monitoring of aircraft via piezoelectric actuator/sensor patches. In Smart Structures \& Materials' 95 (pp. 268-276). International Society for Optics and Photonics.

110 Koh, Y. L., Rajic, N., Chiu, W. K., \& Galea, S. (1999). Smart structure for composite repair. Composite structures, 47(1), 745-752.

111 Roach, D. W., \& Rackow, K. A. (2006). Health monitoring of aircraft structures using distributed sensor systems (No. SAND2006-0834C). Sandia National Laboratories.

112 Park, G., \& Inman, D. J. (2007). Structural health monitoring using piezoelectric impedance measurements. Philosophical Transactions of the Royal Society A: Mathematical, Physical and Engineering Sciences, 365(1851), 373-392.

113 Oborski, P. (2014). Developments in integration of advanced monitoring systems. The International Journal of Advanced Manufacturing Technology, 75(9-12), 1613-1632.

114 Lauro, C. H., Brandão, L. C., Baldo, D., Reis, R. A., \& Davim, J. P. (2014). Monitoring and processing signal applied in machining processes-A review. Measurement, 58, 73-86.

115 Zagrai, A. N., \& Giurgiutiu, V. (2001). Electro-mechanical impedance method for crack detection in thin plates. Journal of Intelligent Material Systems and Structures, 12(10), 709-718.

116 Yang, Y., Lim, Y. Y., \& Soh, C. K. (2008). Practical issues related to the application of the electromechanical impedance technique in the structural health monitoring of civil structures: I. Experiment. Smart Materials and Structures, 17(3), 035008.

117 Yu, L., Bao, J., \& Giurgiutiu, V. (2004, July). Signal processing techniques for damage detection with piezoelectric wafer active sensors and embedded ultrasonic structural radar. In Smart Structures and Materials (pp. 492-503). International Society for Optics and Photonics.

118 Rizzo, P., \& di Scalea, F. L. (2004, July). Discrete wavelet transform to improve guided-wave-based health monitoring of tendons and cables. In Smart Structures and Materials (pp. 523-532). International Society for Optics and Photonics.

119 Kercel, S. W., Klein, M. B., \& Pouet, B. F. (2000, April). Wavelet and waveletpacket analysis of Lamb wave signatures in laser ultrasonics. In AeroSense 2000 (pp. 308-317). International Society for Optics and Photonics. 
120 Hamming, R. W. (1989). Digital filters. Courier Corporation.

121 Staszewski, W. J. (2000). Advanced data pre-processing for damage identification based on pattern recognition. International Journal of Systems Science, 31(11), 1381-1396.

122 Niethammer, M., Jacobs, L. J., Qu, J., \& Jarzynski, J. (2001). Time-frequency representations of Lamb waves. The Journal of the Acoustical Society of America, 109(5), 1841-1847.

123 Staszewski, W. J. (1998). Wavelet based compression and feature selection for vibration analysis. Journal of sound and vibration, 211(5), 735-760.

124 Alleyne, D., \& Cawley, P. (1991). A two-dimensional Fourier transform method for the measurement of propagating multimode signals. The Journal of the Acoustical Society of America, 89(3), 1159-1168.

125 Martinez, L., Morvan, B., \& Izbicki, J. L. (2004). Space-time-wave numberfrequency $\mathrm{Z}(\mathrm{x}, \mathrm{t}, \mathrm{k}, \mathrm{f})$ analysis of SAW generation on fluid filled cylindrical shells. Ultrasonics, 42(1), 383-389.

126 Lin, X., \& Yuan, F. G. (2001). Detection of multiple damages by prestack reverse-time migration. AIAA journal, 39(11), 2206-2215.

127 Sundararaman, S., Adams, D. E., \& Rigas, E. J. (2005). Structural damage identification in homogeneous and heterogeneous structures using beamforming. Structural Health Monitoring, 4(2), 171-190.

128 Giurgiutiu, V., \& Bao, J. (2002, January). Embedded ultrasonic structural radar with piezoelectric wafer active sensors for the NDE of thin-wall structures. In ASME 2002 International Mechanical Engineering Congress and Exposition (pp. 31-38). American Society of Mechanical Engineers.

129 Purekar, A., \& Pines, D. J. (2003, April). Damage interrogation using a phased piezoelectric sensor/actuator array: simulation results on two dimensional isotropic structures. In Proceedings of the 44th AIAA/ASME/ASCE/AHS Structures, Structural Dynamics, and Materials Conference (pp. 7-10).

130 Su, Z., \& Ye, L. (2004). An intelligent signal processing and pattern recognition technique for defect identification using an active sensor network. Smart materials and structures, 13(4), 957. 
131 Challis, R. E., Bork, U., \& Todd, P. C. D. (1996). Ultrasonic NDE of adhered Tjoints using Lamb waves and intelligent signal processing. Ultrasonics, 34(2), 455-459.

132 Legendre, S., Massicotte, D., Goyette, J., \& Bose, T. K. (2001). Neural classification of Lamb wave ultrasonic weld testing signals using wavelet coefficients. Instrumentation and Measurement, IEEE Transactions on, 50(3), 672-678.

133 Zhao, X., Kwan, C., Xu, R., Qian, T., Hay, T., Rose, J. L., ... \& Hexemer, R. (2004, February). Non - Destructive Inspection of Metal Matrix Composites Using Guided Waves. In QUANTITATIVE NONDESTRUCTIVE EVALUATION (Vol. 700, No. 1, pp. 914-921). AIP Publishing.

134 Lin, M., Qing, X., Kumar, A., \& Beard, S. J. (2001, June). Smart layer and smart suitcase for structural health monitoring applications. In SPIE's 8th Annual International Symposium on Smart Structures and Materials (pp. 98-106). International Society for Optics and Photonics.

135 Gorinevsky, D., Gordon, G.A., Beard, S., Kumar, A., and Chang F.-K. (2005). Design of Integrated SHM System for Commercial Air- craft Applications, Proceedings of the $5^{\text {th }}$ International Workshop on Structural Health Monitoring, Stanford, CA, 881-888.

136 Kim, H., and Lee, K. (2005). Diagnostic Network Patch System for SHM and Intelligent Infrastructure, in Proceedings of the SEM International Modal Analysis Conference XXIII, Orlando, FL, Paper 274.

137 Lynch, J. P., Sundararajan, A., Law, K. H., Kiremidjian, A. S., \& Carryer, E. (2004). Embedding damage detection algorithms in a wireless sensing unit for operational power efficiency. Smart Materials and Structures, 13(4), 800.

138 Fekrmandi, H., Gonzalez, R., Rojas, S., Tansel, I. N., Meiller, D., Lindsay K. (2015, June). Automation of the Interpretation of Surface Response to Excitation (SuRE) Method by using Neural Networks. In Recent Advances in Space Technologies (RAST), $20157^{\text {th }}$ International Conference on. IEEE. [In progress]

139 Brigham, E. O., \& Brigham, E. O. (1988). The fast Fourier transform and its applications (Vol. 1). Englewood Cliffs, NJ: Prentice Hall.

140 Vakman, D. (1996). On the analytic signal, the Teager-Kaiser energy algorithm, and other methods for defining amplitude and frequency. IEEE Transactions on Signal Processing, 44(4), 791-797. 
141 Winkelmann, C., Lestari, W., \& La Saponara, V. (2011). Composite structural health monitoring through use of embedded PZT sensors. Journal of Intelligent Material Systems and Structures, 1045389X11406303. 
VITA

\section{HADI FEKRMANDI}

2001-2006

B.Sc., Mechanical Engineering

University of Tabriz, Tabriz, Iran

2006-2009

M.Sc., Mechanical Engineering

University of Tabriz, Tabriz, Iran

2011-2015

$\mathrm{PhD}$, Mechanical Engineering

Florida International University, Miami, FL, USA

\section{PUBLICATIONS AND SELECTED PRESENTATIONS}

Fekrmandi, H., Rojas, J., Tansel, I. N., Yapici, A., \& Uragun, B. (2015). Investigation of the computational efficiency and validity of the surface response to excitation method. Elsevier, Measurement, 62, 33-40.

Fekrmandi, Hadi, et al. "Inspection of the Integrity of a Multi-Bolt Robotic Arm Using a Scanning Laser Vibrometer and Implementing the Surface Response to Excitation Method (SuRE)." International Journal of Prognostics and Health Management 5.1 (2014): 1-10.

Hadi · Fekrmandi · Ibrahim Nur Tansel · Kathleen Oyola • Abdullah Alsenawi "A noncontact method for part-based process performance monitoring in end milling operations”, International Journal of Advanced Manufacturing Technology, Springer Publications. [Accepted]

Hadi Fekrmandi · Amin Baghalian · Sohail Reddy · Ibrahim Nur Tansel • “An Experimental and Numerical Investigation of Surface Response to Excitation Method for automation of machining process monitoring”, Elsevier, Robotics and ComputerIntegrated Manufacturing, Elsevier. [In progress]

Hadi Fekrmandi, Ibrahim Nur Tansel, Rafael Gonzalez Sebastian Rojas, David Meiller, Kyle Lindsay, Amin Baghalian, Shervin Tashakori, Balemir Uragun "Implementation of the Surface Response to Excitation Method (SuRE) With DSPs for detection of damage of this blocks" Proceedings of the $10^{\text {th }}$ International Workshop on Structural Health Monitoring: a Roadmap to Intelligent Structures: September 1-3, 2015, Stanford University. [Accepted]

Hadi Fekrmandi, Ibrahim Nur Tansel, Sebastian Rojas, " Surface Response to Excitation 
Method for Structural Health Monitoring Using Neural Networks." Recent Advances in Space Technologies, 2007. RAST 2015. $7^{\text {th }}$ International Conference on. IEEE, 2015. [In progress]

Hadi Fekrmandi - Ibrahim Nur Tansel "Laser Scanning Vibrometer for Remote Process Performance Monitoring of Automated Manufacturing Operations”, ASME Early Career Technical Conference Proceedings, ECTC 2014, Vol. 13, pages 55-60.

Hadi Fekrmandi · Nikko Miniello · Robel Kiflemariam · Ibrahim Nur Tansel, "COMSOL for Simulation of Surface Response to Excitation Method for Manufacturing Process Performance Monitoring”, COMSOL Conference 2014 Boston, October 8 - 10, 2014, Boston Marriott Newton.

Hadi Fekrmandi · Gurunath Ramanathan - Ibrahim Nur Tansel "Automation of manufacturing process monitoring for CNC milling machines using a remote sensing method based on high-frequency surface guided waves, 27th Florida Conference on Recent Advances in Robotics, FCRAR 2014, May 8-9, Florida International University, Miami, Florida.

Hadi Fekrmandi - Cesar Levy · Qin Ma · Mordechai Perl "An Approximate Formula for the Maximal SIF for an Infinite Array of Longitudinal Coplanar Internal Surface Cracks in an Autofrettaged Cylindrical Pressure Vessel", 4th International Congress on Computational Engineering and Sciences, May 19-24, 2013, Las Vegas, Nevada

Hadi Fekrmandi · Javier Rojas · Michael Wolff · Ibrahim Nur Tansel · Sergio Gonzalez • Balemir Uragun "Monitoring The Health of a Beam Remotely by Using Scanning Laser Vibrometer" ASME Early Career Technical Conference Proceedings, ECTC 2013, Vol 12, pp 302-306.

Sergio Gonzalez · Javier Rojas · Hadi Fekrmandi, Ibrahim Nur Tansel, Balemir Uragun "Implementing the Surface Response to Excitation Method (SuRE) With Non-Contact Sensor at Hard to Reach Locations" Proceedings of the 9th International Workshop on Structural Health Monitoring: a Roadmap to Intelligent Structures: September 10-13, 2013, Stanford University.

Hadi Fekrmandi · David Quintero · Javier Rojas, Jason Campbell · Ibrahim Nur Tansel "Development of a Snake Robot for Structural Health Monitoring of Pipe Structures Using SuRE Method" 26th Florida Conference on Recent Advances in Robotics and Robot Showcase, FCRAR 2013, May 16-17, Florida State University, Tallahassee, Florida. 